\title{
Precise calculation of the decay rate of false vacuum with multi-field bounce
}

\author{
So Chigusa, ${ }^{a}$ Takeo Moroi $^{b}$ and Yutaro Shoji ${ }^{c}$ \\ ${ }^{a}$ KEK Theory Center, IPNS, KEK, \\ Tsukuba, Ibaraki 305-0801, Japan \\ ${ }^{b}$ Department of Physics, The University of Tokyo, \\ Tokyo 113-0033, Japan \\ ${ }^{c}$ Kobayashi-Maskawa Institute for the Origin of Particles and the Universe, Nagoya University, \\ Nagoya, Aichi 464-8602, Japan \\ E-mail: schigusa@post.kek.jp, moroi@phys.s.u-tokyo.ac.jp, \\ yshoji@kmi.nagoya-u.ac.jp
}

ABSTRACT: We study the decay rate of a false vacuum in gauge theory at the one-loop level. We pay particular attention to the case where the bounce consists of an arbitrary number of scalar fields. With a multi-field bounce, which has a curved trajectory in the field space, the mixing among the gauge fields and the scalar fields evolves along the path of the bounce in the field space and the one-loop calculation of the vacuum decay rate becomes complicated. We consider the one-loop contribution to the decay rate with an arbitrary choice of the gauge parameter, and obtain a gauge invariant expression of the vacuum decay rate. We also give proper treatments of gauge zero modes and renormalization.

Keywords: Beyond Standard Model, Higgs Physics

ArXiv ePrint: 2007.14124 


\section{Contents}

1 Introduction 1

2 Setup and formulation 3

2.1 Lagrangian and bounce 3

$\begin{array}{lll}2.2 & \text { Fluctuation operators } & 6\end{array}$

2.2.1 FP ghosts $\quad 8$

$\begin{array}{lll}2.2 .2 & \text { Gauge bosons and scalars } & 8\end{array}$

$\begin{array}{lll}2.3 & \text { Prefactor and functional determinant } & 10\end{array}$

3 Decomposition of solutions $\quad 11$

$\begin{array}{lll}3.1 \quad \ell>0 & 12\end{array}$

$3.2 \ell=0 \quad 13$

4 Functional determinants $\quad 13$

$\begin{array}{lll}4.1 & \ell>0 & 13\end{array}$

$\begin{array}{lll}4.2 \ell=0 & 18\end{array}$

$\begin{array}{lll}4.3 & \text { Background gauge } & 20\end{array}$

5 Zero modes 23

5.1 General issues 23

$\begin{array}{ll}5.2 \text { Gauge zero modes } & 27\end{array}$

5.3 Translational zero modes 30

6 Semi-analytic expression of the decay rate 32

6.1 Contributions of FP ghosts and transverse modes 32

6.2 Contributions of $(S L \varphi)$ modes 33

6.3 Background gauge 34

$\begin{array}{lll}7 & \text { Renormalization } & 35\end{array}$

$\begin{array}{lll}8 & \text { Conclusions and discussion } & 37\end{array}$

$\begin{array}{ll}\text { A Evaluation of determinants } & 38\end{array}$

$\begin{array}{lll}\text { A.1 Alternative fluctuation operators } & 38\end{array}$

A.2 Evaluation of solutions for $\ell>0 \quad 40$

$\begin{array}{lll}\text { A.2.1 Behavior at infinity } & 40\end{array}$

A.2.2 Translational zero modes 42

A.2.3 Functional determinant $(\ell>1) \quad 43$

A.2.4 Functional determinant $(\ell=1) \quad 44$

A.3 Evaluation of solutions for $\ell=0 \quad 45$

$\begin{array}{lll}\text { A.3.1 Behavior at infinity } & 45\end{array}$ 
$\begin{array}{lll}\text { A.3.2 } & \text { Gauge zero modes } & 47\end{array}$

$\begin{array}{lll}\text { A.3.3 Functional determinant } & 49\end{array}$

B Use of alternative fluctuation opeartors $\quad 50$

$\begin{array}{lll}\text { B.1 General discussion } & 50\end{array}$

B.1.1 Setup $\quad 50$

B.1.2 Recursive formula $\quad 51$

$\begin{array}{lll}\text { B.1.3 Error evaluation formula } & 51\end{array}$

$\begin{array}{lll}\text { B.2 Alternative fluctuation operators } & 54\end{array}$

B.2.1 Extended fluctuation operators $\quad 54$

$\begin{array}{lll}\text { B.2.2 Linear approximation } & 56\end{array}$

$\begin{array}{lll}\text { B.3 }(S L \varphi) \text { modes } & 58\end{array}$

$\begin{array}{ll}\text { B.4 }(c \bar{c}) \text { modes } & 59\end{array}$

$\begin{array}{ll}\text { B.5 }(\eta \lambda) \text { modes } & 59\end{array}$

$\begin{array}{ll}\text { C } \overline{\mathrm{MS}} \text { Counterterms } & 60\end{array}$

\section{Introduction}

The decay of a false vacuum has attracted theoretical and phenomenological interests in particle physics and cosmology. For example, in the standard model (SM) and models beyond the SM, there may exist a vacuum whose energy density is lower than that of the electroweak (EW) vacuum. If this is the case, the EW vacuum becomes a false vacuum and is not absolutely stable. Thus, the longevity of the EW vacuum often provides an important constraint on model parameters. In particular, assuming that the standard model is valid up to the Planck scale, the EW vacuum decays within a timescale shorter than the present cosmic age if the top-quark mass is too large or the Higgs mass is too small [1-12]. ${ }^{1}$ In addition, the decay of the false vacuum is also important for the studies of phase transitions in cosmological history, which may be related to inflation or the baryon asymmetry of the Universe. Thus, the precise calculation of the decay rate of the false vacuum is of great importance.

The calculation of a vacuum decay rate has been formulated in [27, 28], where the field configuration called the bounce plays a central role. The bounce is a saddle-point solution of the Euclidean equation of motion, which dominates the path integral for the decay process of the false vacuum. With the bounce configuration being given, the decay rate of a vacuum in unit volume is expressed as

$$
\gamma=\mathcal{A} e^{-\mathcal{B}}
$$

where $\mathcal{B}$ is the action of the bounce and $\mathcal{A}$ contains the effects of the quantum corrections to the action. At the one-loop level, $\mathcal{A}$ is obtained by evaluating the functional determinants

\footnotetext{
${ }^{1}$ For discussion of the absolute stability of the EW vacuum in the SM, see [13-26].
} 
of the fluctuation operators around the false vacuum and those around the bounce. For the precise determination of a vacuum decay rate, the calculation of $\mathcal{A}$ is necessary not only because it fixes the overall factor but also because it cancels out the renormalization scale dependence of $\mathcal{B}$ at the one-loop level [29]. ${ }^{2}$

If scalar fields responsible for the bounce couple to the gauge fields, the fluctuation operator generally depends on the gauge-fixing parameter (which we call $\xi$ ) and $\xi$ appears everywhere in the calculation of the prefactor $\mathcal{A}$. On the other hand, the decay rate of the false vacuum should be independent of $\xi$ because the effective action is gauge independent at its extrema [31, 32]. An explicit check of the gauge invariance at the one-loop level is quite formidable and the first calculation appeared only recently in [33, 34]. In these papers, a manifestly gauge-invariant expression of the decay rate has been obtained for the case where the bounce consists of a single field (single-field bounce). They also address another issue that arises when a gauge symmetry preserved in the false vacuum is broken by the bounce configuration. In such a case, there appears a flat direction of the action corresponding to the global part of the gauge symmetry; it can be seen as a gauge zero mode in the calculation of the functional determinant. Since the fluctuation toward such a flat direction cannot be treated with the saddle point method in the path integral, we need special treatment; a correct prescription for the gauge zero mode has been developed for the single-field bounce [34]. The prescriptions to calculate the decay rate of false vacuum given in $[33,34]$ are essential to perform a complete one-loop calculation of the decay rate of the EW vacuum in the SM [10-12], which update the previous result [1]. ${ }^{3}$ In addition, they are also applied to models beyond the SM $[12,30] .^{4}$

In this paper, we extend the results of $[33,34]$ to the case where the bounce consists of more than one field (multi-field bounce). We give a prescription to obtain a gauge-invariant expression of the vacuum decay rate, adopting two different gauge-fixing conditions; one is the Fermi gauge and the other is the background gauge. In the Fermi gauge, the treatment of the gauge zero mode can be understood easily, but the numerical calculation becomes difficult due to a severe cancellation. On the other hand, in the background gauge, the treatment of the gauge zero mode is complicated, but the numerical calculation becomes easier because of better behavior of fluctuation operators. Thus, we give a prescription to convert the result in the background gauge to that in the Fermi gauge which is guaranteed to be gauge invariant.

This paper is organized as follows. In section 2, we explain our basic setup for the calculation of the vacuum decay rate. We show that the vacuum decay rate (in particular, the prefactor $\mathcal{A}$ ) can be expressed by using solutions of a set of differential equations. In section 3 , we provide a decomposition of the solutions. In section 4, we construct a set

\footnotetext{
${ }^{2}$ The numerical impact of the prefactor is demonstrated in [12, 29, 30].

${ }^{3}$ When the true vacuum appears as a result of the renormalization group improvement of the potential, a careful treatment is required to avoid the double-counting of quantum corrections. If the properties of the bounce are well described by the (renormalizable) Lagrangian with choosing relevant renormalization scale, however, the functional-determinant method can give the one-loop contribution to the prefactor without the double counting; examples include the standard model (see [10-12]).

${ }^{4}$ For other studies about the stability of the electroweak vacuum in models beyond the SM, see, for example, [35-53].
} 
of solutions and calculate their asymptotic behavior, which is needed for the evaluation of $\mathcal{A}$. In section 5 , we provide a general method to treat the zero modes and apply it to the zero modes in association with the gauge and the translational symmetries. In section 6 , we summarize the analytic results. In section 7 , issues related to the renormalization are discussed. Section 8 is devoted to conclusions and discussion.

\section{Setup and formulation}

\subsection{Lagrangian and bounce}

We consider a Euclidean four-dimensional gauge theory with a direct-product gauge group, $\mathfrak{G}$. We concentrate on the contributions from scalar fields and gauge fields; the effects of fermions, if they exist, can be taken into account separately. The Lagrangian is given by

$$
\mathcal{L}_{E}=\frac{1}{4} F_{\mu \nu}^{a} F_{\mu \nu}^{a}+\frac{1}{2}\left(D_{\mu} \phi\right)_{i}\left(D_{\mu} \phi\right)_{i}+V(\phi)+\mathcal{L}^{(\mathrm{GF})}+\mathcal{L}^{(\text {ghost })}
$$

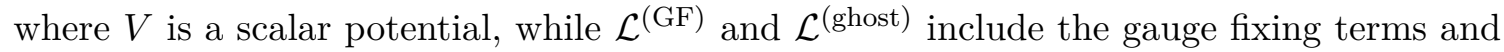
the terms containing the ghosts, respectively, which will be defined later. In addition, $F_{\mu \nu}^{a}$ is the field strength of the gauge field $A_{\mu}^{a}$ (with $a$ being the adjoint index of $\mathfrak{G}$ ), while $\phi_{i}$ (with $i=1-n_{\phi}$ being the index distinguishing scalars) are real scalar fields. ${ }^{5}$ Here and hereafter, the summation over the repeated scalar indices is implicit. The covariant derivative of $\phi$ is defined as

$$
D_{\mu} \phi=\left(\partial_{\mu}+g_{a} A_{\mu}^{a} T^{a}\right) \phi
$$

In the real basis we are working with, the generators satisfy

$$
\begin{aligned}
\left(T^{a}\right)^{T} & =-T^{a}, \\
{\left[T^{a}, T^{b}\right] } & =-f^{a b c} T^{c},
\end{aligned}
$$

where the superscript " $T$ " denotes transpose and $f^{a b c}$ is the structure constant. The gauge coupling constants, denoted as $g_{a}$, can be different for different subgroups of $\mathfrak{G}$. We assume that the scalar potential, $V(\phi)$, has two minima, i.e., the false vacuum and the true vacuum (the false vacuum has higher potential energy than the true vacuum).

For the process of the false vacuum decay, the path integral is dominated by the field configuration called "bounce," an $O(4)$ symmetric saddle-point solution of the Euclidean equations of motion $[54,55]$. Due to the gauge symmetry, there exist an infinite number of solutions. We adopt one solution with $A_{\mu}=0$. Then, the bounce configuration, which we denote as $\bar{\phi}(r)$, satisfies

$$
\partial_{r}^{2} \bar{\phi}_{i}+\frac{3}{r} \partial_{r} \bar{\phi}_{i}=\left.\frac{\partial V}{\partial \phi_{i}}\right|_{\phi \rightarrow \bar{\phi}}
$$

\footnotetext{
${ }^{5}$ In our convention, complex scalar fields are understood to be decomposed into pairs of real scalar fields.
} 
with the following boundary conditions:

$$
\begin{aligned}
\partial_{r} \bar{\phi}_{i}(0) & =0, \\
\bar{\phi}_{i}(\infty) & =v_{i},
\end{aligned}
$$

where $v_{i}$ denotes the scalar amplitude at the false vacuum. Here, $r \equiv \sqrt{x_{\mu} x_{\mu}}$ is the radius from the center of the bounce, and $\partial_{r}$ denotes the derivative with respect to $r$.

The bounce solution has the following properties. First, since the potential is symmetric under the infinitesimal gauge transformation $\phi \rightarrow \phi+\delta \theta^{a} T^{a} \phi$ with an arbitrary choice of transformation parameters $\delta \theta^{a}$,

$$
\frac{\partial V}{\partial \phi_{i}} T_{i j}^{a} \phi_{j}=0
$$

Differentiating it with respect to $\phi_{k}$, we obtain

$$
T_{k i}^{a} \frac{\partial V}{\partial \phi_{i}}=\frac{\partial^{2} V}{\partial \phi_{k} \partial \phi_{i}} T_{i j}^{a} \phi_{j}
$$

Then, from eq. (2.5),

$$
\partial_{r}^{2}\left(T^{a} \bar{\phi}\right)+\frac{3}{r} \partial_{r}\left(T^{a} \bar{\phi}\right)=\Omega\left(T^{a} \bar{\phi}\right)
$$

where

$$
\Omega_{i j}=\left.\frac{\partial^{2} V}{\partial \phi_{i} \partial \phi_{j}}\right|_{\phi \rightarrow \bar{\phi}}
$$

From eq. (2.10), we obtain

$$
\frac{1}{r^{3}} \partial_{r} r^{3}\left[\left(\partial_{r} \bar{\phi}\right)^{T} T^{a} \bar{\phi}\right]=\frac{1}{2} \bar{\phi}^{T}\left[T^{a}, \Omega\right] \bar{\phi}=0,
$$

because $\Omega$ is gauge invariant. It implies that $\left(\partial_{r} \bar{\phi}\right)^{T} T^{a} \bar{\phi}$ should be proportional to $r^{-3}$ or zero. Since it should vanish at the origin,

$$
\left(\partial_{r} \bar{\phi}\right)^{T} T^{a} \bar{\phi}=0
$$

For the later convenience, we define

$$
M_{i a}(r)=-g_{a} T_{i k}^{a} \bar{\phi}_{k}(r)
$$

which satisfies

$$
\left(\partial_{r}^{2}+\frac{3}{r} \partial_{r}-\Omega\right) M=0 .
$$

Using eq. (2.13), the following relation holds:

$$
M^{T} M^{\prime}=\left(M^{T}\right)^{\prime} M,
$$

where $M^{\prime}=\partial_{r} M$. Notice that the gauge boson mass matrix in the false vacuum is given by $M^{T} M(r \rightarrow \infty)$. 
In our analysis, we concentrate on the case where the following conditions hold:

- The rank of the matrix $M^{T} M(r)$ is unchanged for $r<\infty$. (At the false vacuum, some of the broken gauge symmetries may be recovered so that the ranks of $M^{T} M(r<\infty)$ and $M^{T} M(\infty)$ may be different.)

- There are no zero modes except for the gauge zero modes and the translational zero modes.

- At a large $r, M^{T} M(r)$ approaches to $M^{T} M(\infty)$ exponentially.

The second condition is just for simplicity and our results can be extended to the cases with additional zero modes. The third condition is violated when the theory has the (approximate) scale invariance, which has already been discussed for the single-field case [10-12] and the multi-field case [30].

We define the subset of gauge fields that acquire masses from the bounce at $r<\infty$. Then, we define the following numbers:

- $n_{G}$ : the number of the gauge bosons which acquire masses from the bounce at $r<\infty$.

- $n_{B}$ : the number of the gauge bosons which remain massive at the false vacuum.

- $n_{U}$ : the number of the gauge bosons which become massless at the false vacuum (but are massive at $r<\infty)$.

- $n_{\varphi}$ : the total number of scalar fields.

- $n_{H}$ : the number of the scalar fields that do not mix with the gauge bosons at $r<\infty$.

Notice that $n_{\varphi}=n_{G}+n_{H}$ and $n_{G}=n_{B}+n_{U}$.

Now we introduce the gauge fixing terms. We consider the following two gauge fixing conditions:

1. Fermi gauge:

$$
\begin{aligned}
\mathcal{L}_{\text {Fermi }}^{(\mathrm{GF})} & =\frac{1}{2 \xi}\left(\partial_{\mu} A_{\mu}^{a}\right)^{2}, \\
\mathcal{L}_{\text {Fermi }}^{\text {(ghost) }} & =-\bar{c} \partial_{\mu} D_{\mu} c .
\end{aligned}
$$

2. Background gauge:

$$
\begin{aligned}
\mathcal{L}_{\mathrm{BG}}^{(\mathrm{GF})} & =\frac{1}{2 \xi}\left(\partial_{\mu} A_{\mu}^{a}+\xi g_{a} \phi^{T} T^{a} \bar{\phi}\right)^{2}, \\
\mathcal{L}_{\mathrm{BG}}^{\text {(ghost) }} & =\bar{c}^{a}\left[-\partial_{\mu} D_{\mu}^{a b}+\xi\left(g_{a} T^{a} \bar{\phi}\right)^{T}\left(g_{b} T^{b} \phi\right)\right] c^{b} .
\end{aligned}
$$

These gauge fixing terms are consistent with the bounce of our choice since their contributions to the equation of motion vanish in the limit of $A_{\mu} \rightarrow 0$ and $\phi \rightarrow \bar{\phi}$. They are also consistent with the spacial translational invariance; it corresponds to the fluctuation of 
$\delta \phi=\delta x_{\mu} \partial_{\mu} \bar{\phi}$. As for the global gauge transformation, $\delta \phi=\delta \theta^{a} T^{a} \bar{\phi}$, the gauge fixing term of the Fermi gauge is invariant $[34,56]$. Then, in the Fermi gauge, a prescription to take care of the gauge zero modes is available. On the contrary, in the background gauge, the proper treatment of the gauge zero modes becomes more complicated, as we will discuss. The background gauge is, however, useful for numerical calculation especially with $\xi=1$. Thus, we also discuss how the prefactors based on these two gauge fixing conditions are related in this paper.

\section{$2.2 \quad$ Fluctuation operators}

To evaluate the decay rate of the false vacuum, we integrate out the fluctuations around the bounce configuration. As is explicitly given in the next subsection, the prefactor is expressed with the functional determinant of the fluctuation operators, which are the second derivative of the action with respect to the fluctuations.

In discussing the contributions to the prefactor in our setup, we should consider the fluctuation operator for the bosons and that for the Faddeev-Popov (FP) ghosts. In the Fermi gauge, they are given by ${ }^{6}$

$$
\mathcal{M}^{\left(A_{\mu} \varphi\right)}=\left(\begin{array}{cc}
\left(-\partial^{2}+M^{T} M\right) \delta_{\mu \nu}+\left(1-\frac{1}{\xi}\right) \partial_{\mu} \partial_{\nu} & \left(\partial_{\nu} M\right)^{T}-M^{T} \partial_{\nu} \\
2\left(\partial_{\mu} M\right)+M \partial_{\mu} & -\partial^{2}+\Omega
\end{array}\right),
$$

and

$$
\mathcal{M}^{(c \bar{c})}=-\partial^{2}
$$

respectively. Note that $\mathcal{M}^{\left(A_{\mu} \varphi\right)}$ is an $\left(n_{G}+n_{\varphi}\right) \times\left(n_{G}+n_{\varphi}\right)$ object and $\mathcal{M}^{(c \bar{c})}$ is $n_{G} \times n_{G}$. In addition, in the background gauge,

$$
\begin{aligned}
\mathcal{M}_{\mathrm{BG}}^{\left(A_{\mu} \varphi\right)} & =\left(\begin{array}{cc}
\left(-\partial^{2}+M^{T} M\right) \delta_{\mu \nu}+\left(1-\frac{1}{\xi}\right) \partial_{\mu} \partial_{\nu} & 2\left(\partial_{\nu} M\right)^{T} \\
2\left(\partial_{\mu} M\right) & -\partial^{2}+\Omega+\xi M M^{T}
\end{array}\right), \\
\mathcal{M}_{\mathrm{BG}}^{(c \bar{c})} & =-\partial^{2}+\xi M^{T} M .
\end{aligned}
$$

In order for the following discussion, let us define

$$
\begin{aligned}
\widehat{M} & \equiv M(r \rightarrow \infty), \\
\widehat{\Omega} & \equiv \Omega(r \rightarrow \infty) .
\end{aligned}
$$

Then, using eq. (2.15) as well as $M^{\prime}(r \rightarrow \infty)=0$, we obtain

$$
\widehat{\Omega} \widehat{M}=0 .
$$

\footnotetext{
${ }^{6}$ We do not put the subscript "Fermi" on the fluctuation operators in the Fermi gauge for notational simplicity while we put the subscript "BG" on those in the background gauge.
} 
For later convenience, we choose the following basis of the gauge bosons and the scalars:

$$
\begin{aligned}
& \widehat{M}=\left(\begin{array}{cc}
\widehat{W} & 0 \\
0 & 0
\end{array}\right)\left\{\begin{array}{l}
n_{B} \\
n_{B}+n_{H},
\end{array} n_{n_{U}}\right. \\
& \widehat{\Omega}=\left(\begin{array}{cc}
0 & 0 \\
0 & \widehat{m}^{2}
\end{array}\right)\left\{\begin{array}{l}
n_{B} \\
n_{U}+n_{H}
\end{array}\right.
\end{aligned}
$$

where $\widehat{W}$ and $\widehat{m}^{2}$ are full rank diagonal matrices. ${ }^{7}$ Such a choice always exists because of eq. (2.27). In this basis, we also define submatrices of $M$ as

$$
M(r)=(\underbrace{M_{B}(r)}_{n_{B}} \underbrace{M_{U}(r)}_{n_{U}}) .
$$

In addition, we define

$$
M_{0} \equiv M(r=0)
$$

The fluctuation around the bounce can be expanded using the hyperspherical functions on $S^{3}$, which are functions of $\hat{x}_{\mu} \equiv x_{\mu} / r$. The rotational Lie algebra of the four dimensional Euclidean space is equivalent to $\mathrm{SU}(2)_{L} \times \mathrm{SU}(2)_{R}$ and the hyperspherical functions, $\mathcal{Y}_{P}\left(\hat{x}_{\mu}\right)$, are labeled by indices $P=\left(\ell, m_{A}, m_{B}\right)$. Here, $\ell=0,1,2, \cdots$ is the azimuthal quantum number and $m_{A}$ and $m_{B}$ are the magnetic quantum numbers that take values of $-\ell / 2, \ell / 2+$ $1, \cdots, \ell / 2 .{ }^{8}$ Using mode functions that depend only on $r$ and the hyperspherical functions, we expand fields around the bounce as

$$
\begin{aligned}
\phi(x)= & \bar{\phi}(r)+\alpha_{\varphi}^{P}(r) \mathcal{Y}_{P}, \\
A_{\mu}^{a}(x)= & \alpha_{S}^{a P}(r) \frac{x_{\mu}}{r} \mathcal{Y}_{P}+\alpha_{L}^{a P}(r) \frac{r}{L} \partial_{\mu} \mathcal{Y}_{P} \\
& +\alpha_{T 1}^{a P}(r) i \epsilon_{\mu \nu \rho \sigma} V_{\nu}^{(1)} L_{\rho \sigma} \mathcal{Y}_{P}+\alpha_{T 2}^{a P}(r) i \epsilon_{\mu \nu \rho \sigma} V_{\nu}^{(2)} L_{\rho \sigma} \mathcal{Y}_{P}, \\
c^{a}(x)= & \alpha_{c}^{a P}(r) \mathcal{Y}_{P} \\
\bar{c}^{a}(x)= & \alpha_{\bar{c}}^{a P}(r) \mathcal{Y}_{P}
\end{aligned}
$$

where the summation over $P$ is implicit. Here, $V_{\nu}^{(1)}$ and $V_{\nu}^{(2)}$ are arbitrary two independent vectors and

$$
\begin{aligned}
L_{\rho \sigma} & =\frac{i}{\sqrt{2}}\left(x_{\rho} \partial_{\sigma}-x_{\sigma} \partial_{\rho}\right), \\
L & =\sqrt{\ell(\ell+2)} .
\end{aligned}
$$

\footnotetext{
${ }^{7}$ We consider the case where there is no massless physical scalar in the false vacuum, and hence $\hat{m}^{2}$ does not have zero eigenvalues.

${ }^{8}$ The variable $J$ used in $[33,34]$ and $\ell$ are related as $\ell=2 J$.
} 
Note that $(L)$ and $(T)$ modes do not have $\ell=0$ mode. For the following discussion, it is convenient to define the derivative operator that corresponds to the Laplacian acting on the modes with the azimuthal quantum number $\ell$ :

$$
\Delta_{\ell}=\partial_{r}^{2}+\frac{3}{r} \partial_{r}-\frac{L^{2}}{r^{2}}
$$

Similarly, we define the fluctuation operators at the false vacuum, which can be obtained by replacement $M \rightarrow \widehat{M}$ and $\Omega \rightarrow \widehat{\Omega}$. We denote them as $\widehat{\mathcal{M}}^{\left(A_{\mu} \varphi\right)}$ and $\widehat{\mathcal{M}}^{(c \bar{c})}$.

In the following, we will show the hyperspherical expansion of the fluctuation operators around the bounce and around the false vacuum.

\subsubsection{FP ghosts}

The fluctuations of the FP ghosts can be expanded by using the hyperspherical functions. Correspondingly, the fluctuation operator for the FP ghosts can be block-diagonalized as

$$
\mathcal{M}^{(c \bar{c})}=\bigoplus_{\ell=0}^{\infty}\left(\mathcal{M}_{\ell}^{(c \bar{c})}\right)^{2(\ell+1)^{2}}
$$

where the power comes from $(\ell+1)^{2}$ different choices of $\left(m_{A}, m_{B}\right)$ and the complexity of the FP ghosts. In the Fermi gauge,

$$
\mathcal{M}_{\ell}^{(c \bar{c})}=-\Delta_{\ell}
$$

while in the background gauge,

$$
\mathcal{M}_{\ell, \mathrm{BG}}^{(c \bar{c})}=-\Delta_{\ell}+\xi M^{T} M .
$$

Notice that the fluctuation operators for the ghosts are $n_{G} \times n_{G}$ objects.

At the false vacuum, we have a similar block-diagonalization:

$$
\widehat{\mathcal{M}}^{(c \bar{c})}=\bigoplus_{\ell=0}^{\infty}\left(\widehat{\mathcal{M}}_{\ell}^{(c \bar{c})}\right)^{2(\ell+1)^{2}}
$$

where, in the Fermi gauge,

$$
\widehat{\mathcal{M}}_{\ell}^{(c \bar{c})}=-\Delta_{\ell}
$$

while in the background gauge,

$$
\widehat{\mathcal{M}}_{\ell, \mathrm{BG}}^{(c \bar{c})}=-\Delta_{\ell}+\xi \widehat{M}^{T} \widehat{M}
$$

\subsubsection{Gauge bosons and scalars}

Due to the mixing between the gauge bosons and the scalars, we cannot discuss their effects separately. Since only the mode functions with the same $P=\left(\ell, m_{A}, m_{B}\right)$ mix, the fluctuation operator $\mathcal{M}^{\left(A_{\mu} \varphi\right)}$ can be block-diagonalized as

$$
\mathcal{M}^{\left(A_{\mu} \varphi\right)}=\mathcal{M}_{0}^{(S \varphi)} \oplus\left[\bigoplus_{\ell=1}^{\infty}\left(\mathcal{M}_{\ell}^{(S L \varphi)}\right)^{(\ell+1)^{2}}\right] \oplus\left[\bigoplus_{\ell=1}^{\infty}\left(\mathcal{M}_{\ell}^{(T)}\right)^{2(\ell+1)^{2}}\right] .
$$


Here, independently of the choice of the gauge fixing, the fluctuation operator for the $(T)$ modes is given by

$$
\mathcal{M}_{\ell}^{(T)}=-\Delta_{\ell}+M^{T} M
$$

Meanwhile, the fluctuation operators of $(S),(L)$, and $(\varphi)$ modes depend on the gauge fixing. In the Fermi gauge,

$$
\begin{aligned}
\mathcal{M}_{0}^{(S \varphi)}= & \left(\begin{array}{ccc}
-\frac{1}{\xi} \Delta_{1}+M^{T} M & \left(M^{\prime}\right)^{T}-M^{T} \partial_{r} \\
2 M^{\prime}+M \frac{1}{r^{3}} \partial_{r} r^{3} & -\Delta_{0}+\Omega
\end{array}\right), \\
\mathcal{M}_{\ell}^{(S L \varphi)}= & \left(\begin{array}{ccc}
-\Delta_{\ell}+\frac{3}{r^{2}}+M^{T} M & -\frac{2 L}{r^{2}} & \left(M^{\prime}\right)^{T}-M^{T} \partial_{r} \\
-\frac{2 L}{r^{2}} & -\Delta_{\ell}-\frac{1}{r^{2}}+M^{T} M & -\frac{L}{r} M^{T} \\
2 M^{\prime}+M \frac{1}{r^{3}} \partial_{r} r^{3} & -\frac{L}{r} M & -\Delta_{\ell}+\Omega
\end{array}\right) \\
& +\left(1-\frac{1}{\xi}\right)\left(\begin{array}{ccc}
\partial_{r}^{2}+\frac{3}{r} \partial_{r}-\frac{3}{r^{2}} & -L\left(\frac{1}{r} \partial_{r}-\frac{1}{r^{2}}\right) & 0 \\
L\left(\frac{1}{r} \partial_{r}+\frac{3}{r^{2}}\right) & -\frac{L^{2}}{r^{2}} & 0 \\
0 & 0 & 0
\end{array}\right) .
\end{aligned}
$$

Note that $\mathcal{M}_{0}^{(S \varphi)}$ is $\left(n_{G}+n_{\varphi}\right) \times\left(n_{G}+n_{\varphi}\right)$, while $\mathcal{M}_{\ell}^{(S L \varphi)}$ is $\left(2 n_{G}+n_{\varphi}\right) \times\left(2 n_{G}+n_{\varphi}\right)$.

Meanwhile, in the background gauge,

$$
\begin{aligned}
\mathcal{M}_{0, \mathrm{BG}}^{(S \varphi)}= & \left(\begin{array}{cc}
-\frac{1}{\xi} \Delta_{1}+M^{T} M & 2\left(M^{\prime}\right)^{T} \\
2 M^{\prime} & -\Delta_{0}+\Omega+\xi M M^{T}
\end{array}\right), \\
\mathcal{M}_{\ell, \mathrm{BG}}^{(S L \varphi)}= & \left(\begin{array}{ccc}
-\Delta_{\ell}+\frac{3}{r^{2}}+M^{T} M & -\frac{2 L}{r^{2}} & 2\left(M^{\prime}\right)^{T} \\
-\frac{2 L}{r^{2}} & -\Delta_{\ell}-\frac{1}{r^{2}}+M^{T} M & 0 \\
2 M^{\prime} & 0 & -\Delta_{\ell}+\Omega+\xi M M^{T}
\end{array}\right) \\
& +\left(1-\frac{1}{\xi}\right)\left(\begin{array}{ccc}
\partial_{r}^{2}+\frac{3}{r} \partial_{r}-\frac{3}{r^{2}} & -L\left(\frac{1}{r} \partial_{r}-\frac{1}{r^{2}}\right) & 0 \\
L\left(\frac{1}{r} \partial_{r}+\frac{3}{r^{2}}\right) & -\frac{L^{2}}{r^{2}} & 0 \\
0 & 0 & 0
\end{array}\right) .
\end{aligned}
$$

At the false vacuum, similar block-diagonalizations hold. For the $(T)$ mode,

$$
\widehat{\mathcal{M}}_{\ell}^{(T)}=-\Delta_{\ell}+\widehat{M}^{T} \widehat{M}
$$

The fluctuation operator for $(S L \varphi)$ modes at the false vacuum can be further blockdiagonalized thanks to the choice of the basis of eqs. (2.28) and (2.29). For $\ell>0$, the fluctuation operator can be expressed as

$$
\widehat{\mathcal{M}}_{\ell}^{(S L \varphi)}=\left.\mathcal{M}_{\ell}^{(S L \varphi)}\right|_{\bar{\phi} \rightarrow v}=\widehat{\mathcal{M}}_{\ell}^{(B)} \oplus \widehat{\mathcal{M}}_{\ell}^{(U)} \oplus \widehat{\mathcal{M}}_{\ell}^{(\sigma)},
$$

corresponding to the contributions from massive gauge bosons and Nambu-Goldstone (NG) bosons (due to broken gauge symmetry), massless gauge bosons (in association with unbroken gauge symmetry), and physical scalars. Firstly, contributions to $\widehat{\mathcal{M}}_{\ell}^{(B)}$ are from $(S)$ and $(L)$ modes of gauge bosons corresponding to broken symmetries $\left(a=1, \ldots, n_{B}\right)$ and 
the corresponding NG modes $\left(i=1, \ldots, n_{B}\right)$. Then, $\widehat{\mathcal{M}}_{\ell}^{(B)}$ is a $3 n_{B} \times 3 n_{B}$ object and is given by

$$
\begin{aligned}
\widehat{\mathcal{M}}_{\ell}^{(B)}= & \left(\begin{array}{ccc}
-\Delta_{\ell}+\frac{3}{r^{2}}+\widehat{W}^{T} \widehat{W} & -\frac{2 L}{r^{2}} & -\widehat{W}^{T} \partial_{r} \\
-\frac{2 L}{r^{2}} & -\Delta_{\ell}-\frac{1}{r^{2}}+\widehat{W}^{T} \widehat{W} & -\frac{L}{r} \widehat{W}^{T} \\
\widehat{W} \frac{1}{r^{3}} \partial_{r} r^{3} & -\frac{L}{r} \widehat{W} & -\Delta_{\ell}
\end{array}\right) \\
& +\left(1-\frac{1}{\xi}\right)\left(\begin{array}{ccc}
\partial_{r}^{2}+\frac{3}{r} \partial_{r}-\frac{3}{r^{2}} & -L\left(\frac{1}{r} \partial_{r}-\frac{1}{r^{2}}\right) & 0 \\
L\left(\frac{1}{r} \partial_{r}+\frac{3}{r^{2}}\right) & -\frac{L^{2}}{r^{2}} & 0 \\
0 & 0 & 0
\end{array}\right)
\end{aligned}
$$

Secondly, contributions to $\widehat{\mathcal{M}}_{\ell}^{(U)}$ are from massless gauge bosons in $(S)$ and $(L)$ modes. We obtain a $2 n_{U} \times 2 n_{U}$ fluctuation operator as

$$
\widehat{\mathcal{M}}_{\ell}^{(U)}=\left(\begin{array}{cc}
-\Delta_{\ell}+\frac{3}{r^{2}} & -\frac{2 L}{r^{2}} \\
-\frac{2 L}{r^{2}} & -\Delta_{\ell}-\frac{1}{r^{2}}
\end{array}\right)+\left(1-\frac{1}{\xi}\right)\left(\begin{array}{c}
\partial_{r}^{2}+\frac{3}{r} \partial_{r}-\frac{3}{r^{2}}-L\left(\frac{1}{r} \partial_{r}-\frac{1}{r^{2}}\right) \\
L\left(\frac{1}{r} \partial_{r}+\frac{3}{r^{2}}\right) \\
-\frac{L^{2}}{r^{2}}
\end{array}\right)
$$

For the other massive scalars,

$$
\widehat{\mathcal{M}}_{\ell}^{(\sigma)}=-\Delta_{\ell}+\widehat{m}^{2}
$$

which is an $\left(n_{U}+n_{H}\right) \times\left(n_{U}+n_{H}\right)$ object.

Similarly, for $\ell=0$, we obtain

$$
\widehat{\mathcal{M}}_{0}^{(S \varphi)}=\widehat{\mathcal{M}}_{0}^{(B)} \oplus \widehat{\mathcal{M}}_{0}^{(U)} \oplus \widehat{\mathcal{M}}_{0}^{(\sigma)}
$$

where

$$
\begin{aligned}
\widehat{\mathcal{M}}_{0}^{(B)} & =\left(\begin{array}{cc}
-\frac{1}{\xi} \Delta_{1}+\widehat{W}^{T} \widehat{W} & -\widehat{W}^{T} \partial_{r} \\
\widehat{W} \frac{1}{r^{3}} \partial_{r} r^{3} & -\Delta_{0}
\end{array}\right), \\
\widehat{\mathcal{M}}_{0}^{(U)} & =-\frac{1}{\xi} \Delta_{1} \\
\widehat{\mathcal{M}}_{0}^{(\sigma)} & =-\Delta_{0}+\widehat{m}^{2} .
\end{aligned}
$$

\subsection{Prefactor and functional determinant}

The prefactor $\mathcal{A}$ is expressed as

$$
\mathcal{A}=\mathcal{A}^{(c \bar{c})} \mathcal{A}^{\left(A_{\mu} \varphi\right)}
$$

where $\mathcal{A}^{(c \bar{c})}$ denotes the contributions from the FP ghosts and $\mathcal{A}^{\left(A_{\mu} \varphi\right)}$ denotes those from the gauge bosons and the scalars. For the evaluation of the prefactor at the one-loop level, 
the following quantities are necessary [28]:

$$
\begin{aligned}
\mathcal{A}^{(c \bar{c})}= & \frac{\operatorname{Det} \mathcal{M}^{(c \bar{c})}}{\operatorname{Det} \widehat{\mathcal{M}}^{(c \bar{c})}}=\prod_{\ell=0}^{\infty}\left(\frac{\operatorname{Det} \mathcal{M}_{\ell}^{(c \bar{c})}}{\operatorname{Det} \widehat{\mathcal{M}}_{\ell}^{(c \bar{c})}}\right)^{(\ell+1)^{2}}, \\
\mathcal{A}^{\prime\left(A_{\mu} \varphi\right)}= & {\left[\frac{\operatorname{Det}^{\prime} \mathcal{M}^{\left(A_{\mu} \varphi\right)}}{\operatorname{Det} \widehat{\mathcal{M}}^{\left(A_{\mu} \varphi\right)}}\right]^{-1 / 2} } \\
= & \left(\frac{\operatorname{Det}^{\prime} \mathcal{M}_{0}^{(S \varphi)}}{\operatorname{Det} \widehat{\mathcal{M}}_{0}^{(S \varphi)}}\right)^{-1 / 2}\left(\frac{\operatorname{Det}^{\prime} \mathcal{M}_{1}^{(S L \varphi)}}{\operatorname{Det} \widehat{\mathcal{M}}_{1}^{(S L \varphi)}}\right)^{-2} \\
& \times\left[\prod_{\ell=2}^{\infty}\left(\frac{\operatorname{Det} \mathcal{M}_{\ell}^{(S L \varphi)}}{\operatorname{Det} \widehat{\mathcal{M}}_{\ell}^{(S L \varphi)}}\right)^{-(\ell+1)^{2} / 2}\right]\left[\prod_{\ell=1}^{\infty}\left(\frac{\operatorname{Det} \mathcal{M}_{\ell}^{(T)}}{\operatorname{Det} \widehat{\mathcal{M}}_{\ell}^{(T)}}\right)^{-(\ell+1)^{2}}\right] .
\end{aligned}
$$

Here and hereafter, the "prime" is used for quantities after the proper subtraction of the zero modes if necessary. In particular, because of the translational invariance, the $\ell=1$ contribution inevitably contains the effects of translational zero modes [28]. In addition, if $n_{U}>0$, both $\mathcal{M}_{0}^{(S \varphi)}$ and $\mathcal{M}_{0, \mathrm{BG}}^{(S \varphi)}$ have zero eigenvalues. More details about the zero mode subtraction and the relation between $\mathcal{A}^{\left(A_{\mu} \varphi\right)}$ and $\mathcal{A}^{\prime\left(A_{\mu} \varphi\right)}$ will be explained in section 5 .

To evaluate the ratio of two functional determinants, we adopt the method developed in [57-61]. Let $\mathcal{M}_{\ell}^{(X)}$ and $\widehat{\mathcal{M}}_{\ell}^{(X)}$ be $n \times n$ fluctuation operators. We first prepare $n$ linearly independent $n$-dimensional functions $\psi_{\ell}^{(X)(I)}(r)$ and $\widehat{\psi}_{\ell}^{(X)(I)}(r)$ (with $I=1-n$ ) that satisfy

$$
\begin{aligned}
& \mathcal{M}_{\ell}^{(X)} \psi_{\ell}^{(X)(I)}=0, \\
& \widehat{\mathcal{M}}_{\ell}^{(X)} \widehat{\psi}_{\ell}^{(X)(I)}=0,
\end{aligned}
$$

and are regular at $r \rightarrow 0$. Then, we define $n \times n$ objects $\Psi_{\ell}^{(X)}(r)$ and $\widehat{\Psi}_{\ell}^{(X)}(r)$ as

$$
\begin{aligned}
\Psi_{\ell}^{(X)}(r) & \equiv\left(\psi_{\ell}^{(X)(1)}(r) \cdots \psi_{\ell}^{(X)(n)}(r)\right), \\
\widehat{\Psi}_{\ell}^{(X)}(r) & \equiv\left(\widehat{\psi}_{\ell}^{(X)(1)}(r) \cdots \widehat{\psi}_{\ell}^{(X)(n)}(r)\right),
\end{aligned}
$$

with which the ratio of functional determinants can be evaluated as

$$
\frac{\operatorname{Det} \mathcal{M}_{\ell}^{(X)}}{\operatorname{Det} \widehat{\mathcal{M}}_{\ell}^{(X)}}=\left(\frac{\operatorname{det} \Psi_{\ell}^{(X)}\left(r_{0}\right)}{\operatorname{det} \widehat{\Psi}_{\ell}^{(X)}\left(r_{0}\right)}\right)^{-1}\left(\frac{\operatorname{det} \Psi_{\ell}^{(X)}\left(r_{\infty}\right)}{\operatorname{det} \widehat{\Psi}_{\ell}^{(X)}\left(r_{\infty}\right)}\right),
$$

where $r_{0}$ and $r_{\infty}$ are abbreviations of $r \rightarrow 0$ and $r \rightarrow \infty$, respectively.

Thus, for the evaluation of the prefactor $\mathcal{A}$, we need to understand the asymptotic behavior of $\Psi_{\ell}^{(X)}$ and $\widehat{\Psi}_{\ell}^{(X)}$ (and hence those of $\operatorname{det} \Psi_{\ell}^{(X)}(r)$ and $\operatorname{det} \widehat{\Psi}_{\ell}^{(X)}(r)$ ) at $r \rightarrow 0$ and $r \rightarrow \infty$. In particular, the behavior of $\operatorname{det} \Psi_{\ell}^{(S L \varphi)}$ and $\operatorname{det} \Psi_{0}^{(S \varphi)}$ is non-trivial because of the mixing among $(S),(L)$, and $(\varphi)$ modes. In the following sections, we discuss how we can evaluate those quantities.

\section{Decomposition of solutions}

In this section, we provide a decomposition of the set of solutions $\Psi_{\ell}^{(S L \varphi)}$ and $\Psi_{0}^{(S \varphi)}$, generalizing the results of $[33,34]$. 


\section{$3.1 \ell>0$}

Let us consider the solutions of the following equation in the Fermi gauge:

$$
\mathcal{M}_{\ell}^{(S L \varphi)} \Psi_{\ell}^{(S L \varphi)}=0 .
$$

Because $\mathcal{M}_{\ell}^{(S L \varphi)}$ is $\left(2 n_{G}+n_{\varphi}\right) \times\left(2 n_{G}+n_{\varphi}\right)$, there exist $\left(2 n_{G}+n_{\varphi}\right)$ linearly independent solutions of eq. (3.1).

Solutions of eq. (3.1) can be decomposed by using four functions, $\chi, \eta, \zeta$, and $\lambda$ :

$$
\Psi_{\ell}^{(S L \varphi)}=\left(\begin{array}{c}
\Psi_{\ell}^{(\mathrm{top})} \\
\Psi_{\ell}^{(\mathrm{mid})} \\
\Psi_{\ell}^{(\mathrm{bot})}
\end{array}\right)=\left(\begin{array}{c}
\partial_{r} \chi \\
\frac{L}{r} \chi \\
M \chi
\end{array}\right)+\left(\begin{array}{c}
\left(M^{T} M\right)^{-1}\left[\frac{L}{r} \eta-2\left(M^{\prime}\right)^{T} \lambda\right] \\
\left(M^{T} M\right)^{-1} \frac{1}{r^{2}} \partial_{r} r^{2} \eta \\
\lambda
\end{array}\right)+\left(\begin{array}{c}
{\left[\partial_{r}\left(M^{T} M\right)^{-1}\right] \zeta} \\
0 \\
M\left(M^{T} M\right)^{-1} \zeta
\end{array}\right) .
$$

Here, the functions $\chi, \eta, \zeta$, and $\lambda$ are dependent on the azimuthal quantum number $\ell$; the subscript " $\ell$ " is omitted from these functions for notational simplicity. The shape of $\chi, \eta$, and $\zeta$ is $n_{G} \times\left(2 n_{G}+n_{\varphi}\right)$, while that of $\lambda$ is $n_{\varphi} \times\left(2 n_{G}+n_{\varphi}\right)$; here, the $\left(2 n_{G}+n_{\varphi}\right)$ columns are linearly independent and are distinguished by the boundary conditions at $r=0$.

The evolution of functions $\chi$ and $\zeta$ is governed by the following differential equations:

$$
\begin{aligned}
\mathcal{M}_{\ell}^{(c \bar{c})} \chi= & {\left[\partial_{r}\left(M^{T} M\right)^{-1}\right] \frac{L}{r} \eta-\frac{2}{r^{3}} \partial_{r} r^{3}\left(M^{T} M\right)^{-1}\left(M^{\prime}\right)^{T} \lambda } \\
& -\mathcal{M}_{0}^{(c \bar{c})}\left(M^{T} M\right)^{-1} \zeta-\frac{1}{r^{3}} \partial_{r} r^{3}\left(M^{T} M\right)^{-1} \partial_{r} \zeta+\xi \zeta, \\
\mathcal{M}_{\ell}^{(c \bar{c})} \zeta= & 0
\end{aligned}
$$

while $\eta$ and $\lambda$ satisfy

$$
\begin{aligned}
\Delta_{\ell} \eta= & M^{T} M\left[\eta-\left\{\partial_{r}\left(M^{T} M\right)^{-1}\right\} \frac{1}{r^{2}} \partial_{r} r^{2} \eta\right]-\frac{2 L}{r} M^{\prime T} \lambda+\frac{L}{r} M^{T} M\left[\partial_{r}\left(M^{T} M\right)^{-1}\right] \zeta, \\
\Delta_{\ell} \lambda= & \Omega \lambda-4 M^{\prime}\left(M^{T} M\right)^{-1} M^{\prime T} \lambda+\frac{2 L}{r} M^{\prime}\left(M^{T} M\right)^{-1} \eta-2 M^{\prime}\left(M^{T} M\right)^{-1} \zeta^{\prime} \\
& +M\left[-\frac{2}{r^{3}} \partial_{r} r^{3}\left(M^{T} M\right)^{-1} M^{\prime T} \lambda+\frac{L}{r}\left\{\partial_{r}\left(M^{T} M\right)^{-1}\right\} \eta-\left\{\partial_{r}\left(M^{T} M\right)^{-1}\right\} \zeta^{\prime}\right] .
\end{aligned}
$$

In addition, $\lambda$ satisfies the following constraint:

$$
M^{T} \lambda=0 .
$$

The above constraint is consistent with the evolution equations; one can derive $\Delta_{\ell}\left(M^{T} \lambda\right)=0$, so that eq. (3.7) holds if it is satisfied at $r=0$.

So far, we have discussed in the Fermi gauge. We note here that the decomposition given in eq. (3.2) is also applicable in the background gauge if we replace $\mathcal{M}_{\ell}^{(c \bar{c})}$ in eqs. (3.3) and (3.4) by $\mathcal{M}_{\ell, \mathrm{BG}}^{(c \bar{c})}$ given in eq. (2.41). (In the background gauge, the relation $\mathcal{M}_{\ell, \mathrm{BG}}^{(c \bar{c})}\left(M^{T} \lambda\right)=0$ holds. Thus, the condition $M^{T} \lambda=0$ is again consistent with the evolution equations.) 
From eqs. (3.2)-(3.6), we can derive the following equations:

$$
\begin{aligned}
\mathcal{M}_{\ell}^{(c \bar{c})}\left(r \Psi_{\ell}^{(\mathrm{mid})}\right) & =-\frac{1}{r^{3}} \partial_{r} r^{4} \eta+\xi L \zeta, \\
\Psi_{\ell}^{(\mathrm{top})} & =\frac{1}{L} \partial_{r}\left(r \Psi_{\ell}^{(\mathrm{mid})}\right)-\frac{r}{L} \eta, \\
\Psi_{\ell}^{(\mathrm{bot})} & =\frac{r}{L} M \Psi_{\ell}^{(\text {mid })}-M\left(M^{T} M\right)^{-1} \frac{1}{L r} \partial_{r} r^{2} \eta+\lambda+M\left(M^{T} M\right)^{-1} \zeta .
\end{aligned}
$$

These expressions are useful to derive the asymptotic behaviors of the solutions. Notice that, in the background gauge, eq. (3.8) is replaced by

$$
\mathcal{M}_{\ell, \mathrm{BG}}^{(c \bar{c})}\left(r \Psi_{\ell, \mathrm{BG}}^{(\mathrm{mid})}\right)=-\frac{1}{r^{3}} \partial_{r} r^{4} \eta+\frac{\xi}{r} \partial_{r} r^{2} \eta,
$$

while eqs. (3.9) and (3.10) are unchanged.

\section{$3.2 \ell=0$}

For $\ell=0$ mode, we need solutions of

$$
\mathcal{M}_{0}^{(S \varphi)} \Psi_{0}^{(S \varphi)}=0 .
$$

The solutions can be decomposed as

$$
\Psi_{0}^{(S \varphi)}=\left(\begin{array}{l}
\Psi_{0}^{(\mathrm{top})} \\
\Psi_{0}^{(\mathrm{bot})}
\end{array}\right)=\left(\begin{array}{c}
\partial_{r} \chi \\
M \chi
\end{array}\right)+\left(\begin{array}{c}
-2\left(M^{T} M\right)^{-1}\left(M^{\prime}\right)^{T} \lambda \\
\lambda
\end{array}\right)+\left(\begin{array}{c}
{\left[\partial_{r}\left(M^{T} M\right)^{-1}\right] \zeta} \\
M\left(M^{T} M\right)^{-1} \zeta
\end{array}\right)
$$

where $\chi, \zeta$, and $\lambda$ for $\ell=0$ satisfy eqs. (3.3), (3.4), and (3.6) with replacing $\ell \rightarrow 0$ and $\eta \rightarrow 0$. Notice that now $\chi$ and $\zeta$ are $n_{G} \times\left(n_{G}+n_{\varphi}\right)$ objects and $\lambda$ is $n_{\varphi} \times\left(n_{G}+n_{\varphi}\right)$; the columns correspond to $\left(n_{G}+n_{\varphi}\right)$ independent choices of the boundary conditions at $r=0$. Thus, $\Psi_{0}^{(S \varphi)}$ is an $\left(n_{G}+n_{\varphi}\right) \times\left(n_{G}+n_{\varphi}\right)$ object.

\section{Functional determinants}

In this section, we study the behavior of the functional determinants of the fluctuation operators in the Fermi gauge. Equivalence to the results in the background gauge is also discussed.

\section{$4.1 \ell>0$}

Let us consider the case with $\ell>0$. For the study of the functional determinants, we should first understand the behavior of the solutions of eq. (3.1). For this purpose, we define an $r$-dependent $n_{\varphi} \times n_{H}$ matrix $V_{H}$, which is given by

$$
V_{H}=\left(u_{1} \cdots u_{n_{H}}\right)
$$

with $u_{p}\left(p=1, \cdots, n_{H}\right)$ being zero eigenvectors of $M^{T}$, i.e.,

$$
M^{T} u_{p}=0, \quad u_{p}^{T} u_{p^{\prime}}=\delta_{p p^{\prime}} .
$$


The columns of the solution of eq. (3.1) can be classified into the following three types:

- Type 1: $\eta^{(1)}=\lambda^{(1)}=\zeta^{(1)}=0$.

- Type 2: $\zeta^{(2)} \neq 0$.

- Type 3: $\zeta^{(3)}=0$, with non-vanishing $\eta^{(3)}$ and $\lambda^{(3)}$,

where the superscripts "(1)," "(2)," and "(3)" indicate solutions for the Type 1, 2, and 3, respectively. Note that the Type 1, 2, and 3 solutions include $n_{G}, n_{G}$, and $n_{\varphi}$ independent solutions, respectively. The full matrix of solutions is constructed as

$$
\eta=(\underbrace{\eta^{(1)}}_{n_{G}} \underbrace{\eta^{(2)}}_{n_{G}} \underbrace{\eta^{(3)}}_{n_{\varphi}}),
$$

and similar for $\chi, \lambda$, and $\zeta$. For each type of solutions, the boundary conditions at $r \rightarrow 0$ are imposed as follows.

- Type 1: We take

$$
\chi^{(1)}(r)=r^{\ell} I_{G}
$$

where $I_{G}$ is the $n_{G} \times n_{G}$ unit matrix. Then, from eq. (3.2), we obtain

$$
\Psi_{\ell}^{(S L \varphi)(1)}(r \rightarrow 0) \simeq\left(\begin{array}{c}
\ell r^{\ell-1} I_{G} \\
L r^{\ell-1} I_{G} \\
M r^{\ell}
\end{array}\right)\left\{\begin{array}{l}
n_{G} \\
n_{G} . \\
n_{\varphi}
\end{array}\right.
$$

- Type 2: We take

$$
\zeta^{(2)}(r)=r^{\ell} I_{G}
$$

At $r \rightarrow 0, M^{\prime} \propto r$ and $V_{H} V_{H}^{T} M^{\prime} \propto r^{2}$. Thus, from eq. (3.5) and eq. (3.6), $\eta^{(2)}(r \rightarrow$ $0)=\mathcal{O}\left(r^{\ell+2}\right)$ and $\lambda^{(2)}(r \rightarrow 0)=\mathcal{O}\left(r^{\ell+3}\right)$. Consequently, using eqs. (3.8)-(3.10), we obtain

$$
\Psi_{\ell}^{(S L \varphi)(2)}(r \rightarrow 0) \simeq(\underbrace{\left.\begin{array}{c}
-\frac{\xi}{4} r^{\ell+1} I_{G} \\
-\frac{\xi \ell}{4 L} r^{\ell+1} I_{G} \\
M\left(M^{T} M\right)^{-1} r^{\ell}
\end{array}\right)}_{n_{G}}\} \begin{aligned}
& n_{G} \\
& n_{G} . \\
& n_{\varphi}
\end{aligned}
$$

- Type 3: This type contains two classes of solutions. One is with $\eta^{(3 \eta)}$ and $\lambda^{(3 \eta)}$ which obey the following boundary conditions:

$$
\eta^{(3 \eta)}(r \rightarrow 0) \simeq r^{\ell} I_{G}, \quad \lambda^{(3 \eta)}(r \rightarrow 0) \simeq 0 .
$$


The other is with $\eta^{(3 \lambda)}$ and $\lambda^{(3 \lambda)}$ whose boundary conditions are given by

$$
\eta^{(3 \lambda)}(r \rightarrow 0) \simeq 0, \quad \lambda^{(3 \lambda)}(r \rightarrow 0) \simeq r^{\ell} V_{H}(r \rightarrow 0) .
$$

Combining two classes of solutions, we define $\left(2 n_{G}+n_{H}\right) \times\left(n_{G}+n_{H}\right)$ object $\Psi_{\ell}^{(S L \varphi)(3)}$ whose behavior at $r \rightarrow 0$ is given by

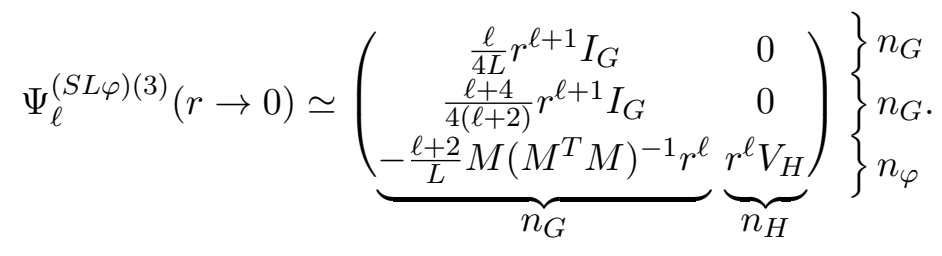

In our convention, because $M^{T} \bar{\phi}^{\prime}=0$, one of $u_{p}$ is chosen to be proportional to $\bar{\phi}^{\prime}$, which we denote as $u^{(\text {tr)} \text { : }}$

$$
u^{(\mathrm{tr})} \equiv \frac{\bar{\phi}^{\prime}}{\sqrt{\bar{\phi}^{\prime T} \bar{\phi}^{\prime}}} .
$$

For $\ell=1$, it is related to the translational zero mode, and is important in eliminating the zero eigenvalues from the functional determinant. The column of $\Psi_{1}^{(S L \varphi)(3)}$ in association with $u^{(\operatorname{tr})}$ is obtained by $\chi^{(\operatorname{tr})}=\eta^{(\operatorname{tr})}=0$ and $\lambda^{(\operatorname{tr})} \propto \bar{\phi}^{\prime}$. We will come back to this issue in section 5 .

The asymptotic behavior at $r \rightarrow \infty$ can be understood by carefully observing the differential equation. We leave the precise discussion to appendix A and, in this section, only show the results. Neglecting terms irrelevant for the calculation of the functional determinant of our interest, the columns of $\Psi_{\ell}^{(S L \varphi)}(r \rightarrow \infty)$ can be obtained by linear combinations of those of the following objects:

$$
\begin{aligned}
& \tilde{\psi}^{(1)}=\underbrace{\left(\begin{array}{c}
\ell r^{\ell-1} I_{G} \\
L r^{\ell-1} I_{G} \\
M r^{\ell}
\end{array}\right)}_{n_{G}}\left\{\begin{array}{l}
n_{G} \\
n_{G}, \\
n_{\varphi}
\end{array}\right. \\
& \tilde{\psi}^{(2)}=\left(\begin{array}{cc}
-\frac{\xi}{4} r^{\ell+1} I_{B} & 0 \\
0 & \frac{\ell-(\ell+2) \xi}{4(\ell+2)} r^{\ell+1} I_{U} \\
-\frac{\xi \ell}{4 L} r^{\ell+1} I_{B} & 0 \\
0 & \frac{\ell[(\ell+4)-(\ell+2) \xi]}{4 L(\ell+2)} r^{\ell+1} I_{U} \\
-\frac{\xi}{4(\ell+2)} r^{\ell+2} M_{B} & \underbrace{0}_{n_{B}}
\end{array}\right\}\left\{\begin{array}{l}
n_{B} \\
n_{U} \\
n_{B} \\
n_{U} \\
n_{\varphi}
\end{array}\right.
\end{aligned}
$$

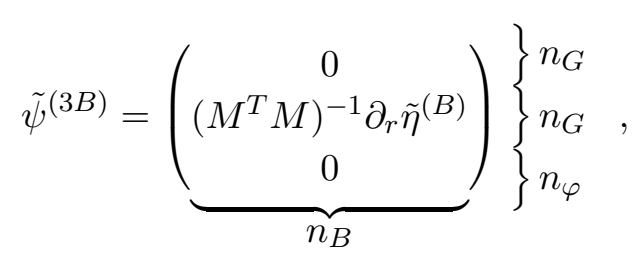




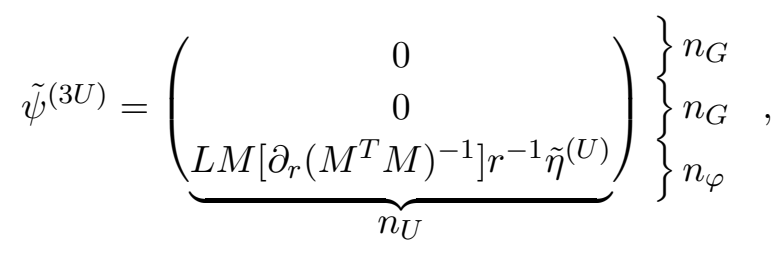

$$
\begin{aligned}
& \tilde{\psi}^{(3 \lambda)}=\underbrace{\left(\begin{array}{c}
0 \\
0 \\
\tilde{\lambda}
\end{array}\right)}_{n_{H}}
\end{aligned}
$$

Here, $I_{B}$ and $I_{U}$ are the $n_{B} \times n_{B}$ and $n_{U} \times n_{U}$ unit matrices, respectively, while $\tilde{\eta}^{(B)}$ and $\tilde{\eta}^{(U)}$ are $n_{G} \times n_{B}$ and $n_{G} \times n_{U}$ objects both of which satisfy

$$
\left\{-\Delta_{\ell}+\left(M^{T} M\right)-\left(M^{T} M\right)\left[\partial_{r}\left(M^{T} M\right)^{-1}\right] \frac{1}{r^{2}} \partial_{r} r^{2}\right\} \tilde{\eta}=0 .
$$

Among the solutions, $\tilde{\eta}^{(B)}$ corresponds to $n_{B}$ solutions that exponentially grow at $r \rightarrow \infty$, while $\tilde{\eta}^{(U)}$ corresponds to $n_{U}$ solutions that behave as $r^{-2}$ at $r \rightarrow \infty$. In addition, $\tilde{\lambda}$ is $n_{\varphi} \times n_{H}$, satisfying

$$
\left(-\Delta_{\ell}+\Omega\right) \tilde{\lambda}=0
$$

and

$$
M^{T} \tilde{\lambda}=0
$$

Then, $\Psi_{\ell}^{(S L \varphi)}(r \rightarrow \infty)$ can be expressed as

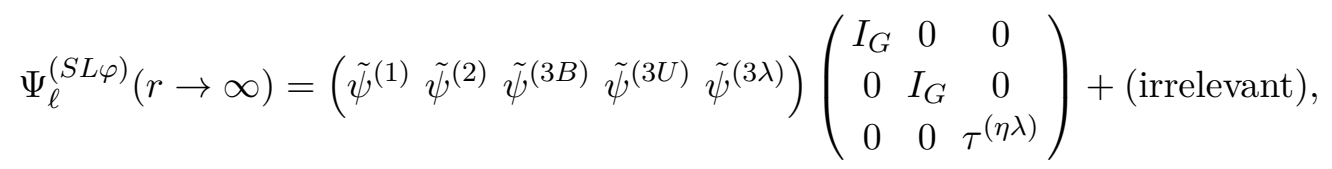

with $\tau^{(\eta \lambda)}$ being an $n_{\varphi} \times n_{\varphi}$ orthogonal matrix. In the above expression, elements irrelevant for the following discussion are neglected.

In order to calculate the prefactor $\mathcal{A}$, we should also consider the fluctuation operator around the false vacuum. In particular, we need to derive solutions of $\widehat{\mathcal{M}}_{\ell}^{(S L \varphi)} \widehat{\Psi}_{\ell}^{(S L \varphi)}=0$. For this purpose, we can use the fact that the fluctuation operator around the false vacuum can be block-diagonalized as eq. (2.52); the blocks are for the fluctuations of massive gauge bosons and NG bosons, for massless gauge bosons, and for physical scalars. Thus, we can discuss their contributions separately. Similarly to the discussion above, we define $\widehat{\Psi}_{\ell}^{(X)}(X=B, U, \sigma)$ obeying $\widehat{\mathcal{M}}_{\ell}^{(X)} \widehat{\Psi}_{\ell}^{(X)}=0$, which describe independent solutions of the differential equation. Note that $\widehat{\Psi}_{\ell}^{(B)}, \widehat{\Psi}_{\ell}^{(U)}$, and $\widehat{\Psi}_{\ell}^{(\sigma)}$ are $3 n_{B} \times 3 n_{B}, 2 n_{U} \times 2 n_{U}$, and $\left(n_{U}+n_{H}\right) \times\left(n_{U}+n_{H}\right)$, respectively. 
Effects of the fluctuations of massive gauge boson and NG bosons around the false vacuum are embedded in $\widehat{\Psi}_{\ell}^{(B)}$, which behaves as

$$
\widehat{\Psi}_{\ell}^{(B)}(r \rightarrow 0)=\left(\begin{array}{ccc}
\ell r^{\ell-1} I_{B} & -\frac{\xi}{4} r^{\ell+1} I_{B} & \frac{\ell}{4 L} r^{\ell+1} I_{B} \\
L r^{\ell-1} I_{B} & -\frac{\xi \ell}{4 L} r^{\ell+1} I_{B} & \frac{\ell+4}{4(\ell+2)} r^{\ell+1} I_{B} \\
\widehat{W} r^{\ell} & \underbrace{\widehat{W}^{-1} r^{\ell}}_{n_{B}} & \underbrace{-\frac{\ell+2}{L} \widehat{W}^{-1} r^{\ell}}_{n_{B}}
\end{array}\right\}\left\{\begin{array}{l}
n_{B} \\
n_{B}, \\
n_{B}
\end{array}\right.
$$

and

$$
\widehat{\Psi}_{\ell}^{(B)}(r \rightarrow \infty)=\left(\begin{array}{ccc}
\ell r^{\ell-1} I_{B} & -\frac{\xi}{4} r^{\ell+1} I_{B} & 0 \\
L r^{\ell-1} I_{B} & -\frac{\xi \ell}{4 L} r^{\ell+1} I_{B} & \widehat{W}^{-1} \widehat{\Psi}_{\ell}^{(\eta)} \\
\widehat{W} r^{\ell} & -\frac{\xi}{4(\ell+2)} r^{\ell+2} \widehat{W} & 0
\end{array}\right)
$$

where $\widehat{\Psi}_{\ell}^{(\eta)}$ is an $n_{B} \times n_{B}$ function satisfying

$$
\left(-\Delta_{\ell}+\widehat{W}^{T} \widehat{W}\right) \widehat{\Psi}_{\ell}^{(\eta)}=0
$$

with

$$
\widehat{\Psi}_{\ell}^{(\eta)}(r \rightarrow 0)=I_{B} r^{\ell}
$$

Next, we consider the fluctuations of massless gauge bosons. For those, we can obtain the solutions in the following form:

$$
\left.\widehat{\Psi}_{\ell}^{(U)}=\left(\begin{array}{ll}
\ell r^{\ell-1} I_{U} & \frac{(\ell+2) \xi-\ell}{4 \ell(\ell+2)} r^{\ell+1} I_{U} \\
\underbrace{\ell-1}_{n_{U}} I_{U} & \underbrace{\frac{(\ell+2) \xi-(\ell+4)}{4 L(\ell+2)} r^{\ell+1} I_{U}}_{n_{U}}
\end{array}\right)\right\} \begin{aligned}
& n_{U} \\
& n_{U}
\end{aligned}
$$

Furthermore, the solutions related to the physical scalars are given by an $\left(n_{U}+n_{H}\right) \times$ $\left(n_{U}+n_{H}\right)$ object, $\widehat{\Psi}_{\ell}^{(\sigma)}$, whose evolution is governed by

$$
\left(-\Delta_{\ell}+\widehat{m}^{2}\right) \widehat{\Psi}_{\ell}^{(\sigma)}=0
$$

with

$$
\widehat{\Psi}_{\ell}^{(\sigma)}(r \rightarrow 0)=\left(\begin{array}{cc}
I_{U} & 0 \\
0 & I_{H}
\end{array}\right) r^{\ell}
$$

where $I_{H}$ is the $n_{H} \times n_{H}$ unit matrix. Then, $\operatorname{det} \widehat{\Psi}_{\ell}^{(S L \varphi)}(r)$ can be calculated as

$$
\operatorname{det} \widehat{\Psi}_{\ell}^{(S L \varphi)}(r)=\operatorname{det}\left(\widehat{\Psi}_{\ell}^{(B)}(r)\right) \operatorname{det}\left(\widehat{\Psi}_{\ell}^{(U)}(r)\right) \operatorname{det}\left(\widehat{\Psi}_{\ell}^{(\sigma)}(r)\right) .
$$

By using $\operatorname{det} \Psi_{\ell}^{(S L \varphi)}$ and $\operatorname{det} \widehat{\Psi}_{\ell}^{(S L \varphi)}$, the functional determinant of our interest can be expressed as

$$
\frac{\operatorname{Det} \mathcal{M}_{\ell}^{(S L \varphi)}}{\operatorname{Det} \widehat{\mathcal{M}}_{\ell}^{(S L \varphi)}}=\left(\frac{\operatorname{det} \Psi_{\ell}^{(S L \varphi)}\left(r_{0}\right)}{\operatorname{det} \widehat{\Psi}_{\ell}^{(S L \varphi)}\left(r_{0}\right)}\right)^{-1}\left(\frac{\operatorname{det} \Psi_{\ell}^{(S L \varphi)}\left(r_{\infty}\right)}{\operatorname{det} \widehat{\Psi}_{\ell}^{(S L \varphi)}\left(r_{\infty}\right)}\right)
$$


In order to evaluate the above quantity, we define

$$
\Psi_{\ell}^{(\eta \lambda)}=\left(\begin{array}{cc}
\eta^{(3 \eta)} & \eta^{(3 \eta)} \\
V_{H}^{T} \lambda^{(3 \lambda)} & V_{H}^{T} \lambda^{(3 \lambda)}
\end{array}\right)
$$

which can be obtained by solving eqs. (3.5) and (3.6) with taking the boundary conditions given in eq. (4.8) or (4.9). Then, by using the following relation:

$$
\operatorname{det} \Psi_{\ell}^{(\eta \lambda)}\left(r_{\infty}\right)=\operatorname{det} \tilde{\eta}\left(r_{\infty}\right) \operatorname{det}\left[V_{H}^{T}\left(r_{\infty}\right) \tilde{\lambda}\left(r_{\infty}\right)\right],
$$

we obtain

$$
\begin{aligned}
\frac{\operatorname{Det} \mathcal{M}_{\ell}^{(S L \varphi)}}{\operatorname{Det} \widehat{\mathcal{M}}_{\ell}^{(S L \varphi)}}= & \left(\frac{\ell}{r_{\infty}}\right)^{n_{U}}\left(\operatorname{det} M^{T} M\right)_{\infty}^{1 / 2}\left[\operatorname{det}\left(M_{U}^{T} M_{U}\right)^{\prime}\right]_{\infty}^{-1} \\
& \left(\operatorname{det} \Psi_{\ell}^{(\eta \lambda)}\left(r_{\infty}\right)\right)\left(\operatorname{det} \widehat{\Psi}_{\ell}^{(\eta)}\left(r_{\infty}\right)\right)^{-1}\left(\operatorname{det} \widehat{\Psi}_{\ell}^{(\sigma)}\left(r_{\infty}\right)\right)^{-1} \\
& \left(\operatorname{det} M_{0}^{T} M_{0}\right)^{1 / 2}\left(\operatorname{det} \widehat{W}^{T} \widehat{W}\right)^{-1}
\end{aligned}
$$

where the subscript " $\infty$ " implies that the quantity should be evaluated at $r=r_{\infty}$; one can check that $r_{0}$ dependence and $r_{\infty}$ dependence cancel out after taking $r_{0} \rightarrow 0$ and $r_{\infty} \rightarrow \infty$. It is important to notice that eq. (4.32) does not contain the gauge parameter, $\xi$.

\section{$4.2 \quad \ell=0$}

Formally, the functional determinant of $\mathcal{M}_{0}^{(S \varphi)}$ can be obtained by a similar procedure as in the case of $\ell>0$. The solutions of $\mathcal{M}_{0}^{(S \varphi)} \Psi_{0}^{(S \varphi)(I)}=0$, corresponding to the Type 1,2 , and 3 solutions, are given in the following form:

\section{- Type 1:}

$$
\Psi_{0}^{(S \varphi)(1)}=\left(\begin{array}{c}
0 \\
M
\end{array}\right)\left\{\begin{array}{l}
n_{G} \\
n_{G}
\end{array}\right.
$$

- Type 2:

$$
\Psi_{0}^{(S \varphi)(2)}=\left(\begin{array}{c}
-\frac{\xi}{4} r I_{G} \\
0
\end{array}\right)\left\{\begin{array}{l}
n_{G} \\
n_{G}
\end{array}+(\text { irrelevant })\right.
$$

where we neglect terms that are irrelevant for the calculation of the functional determinant of our interest.

- Type 3:

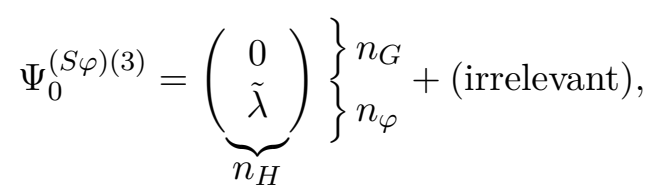

where the function $\tilde{\lambda}$ satisfies eq. (3.6) with taking $\eta \rightarrow 0$ and $\zeta \rightarrow 0$, as well as $M^{T} \tilde{\lambda}=0$. 
Then, we define

$$
\Psi_{0}^{(S \varphi)} \equiv\left(\Psi_{0}^{(S \varphi)(1)} \Psi_{0}^{(S \varphi)(2)} \Psi_{0}^{(S \varphi)(3)}\right)
$$

which will be related to the functional determinant of the fluctuation operator around the bounce.

Recalling the block-diagonalization shown in eq. (2.56), we can solve $\widehat{\mathcal{M}}_{0}^{(X)} \widehat{\Psi}_{0}^{(X)}=0$ $(X=B$ and $U)$ to obtain

$$
\widehat{\Psi}_{0}^{(B)}(r)=(\underbrace{0}_{n_{B}} \underbrace{\left.\begin{array}{c}
0 \frac{1}{4} \xi r I_{B} \\
0
\end{array}\right)}_{n_{B}}\} n_{B},
$$

and

$$
\widehat{\Psi}_{0}^{(U)}(r)=\frac{\xi}{4} r I_{U}
$$

We also define $\widehat{\Psi}_{0}^{(\sigma)}(r)$ that satisfies

$$
\left(\Delta_{0}-\widehat{m}^{2}\right) \widehat{\Psi}_{0}^{(\sigma)}=0
$$

with

$$
\widehat{\Psi}_{0}^{(\sigma)}(r \rightarrow 0)=\left(\begin{array}{cc}
I_{U} & 0 \\
0 & I_{H}
\end{array}\right) .
$$

Using quantities defined above, the determinant is calculated as

$$
\operatorname{det} \widehat{\Psi}_{0}^{(S \varphi)}(r)=\operatorname{det}\left(\widehat{\Psi}_{0}^{(B)}(r)\right) \operatorname{det}\left(\widehat{\Psi}_{0}^{(U)}(r)\right) \operatorname{det}\left(\widehat{\Psi}_{0}^{(\sigma)}(r)\right) .
$$

We obtain the following expression:

$$
\frac{\operatorname{Det} \mathcal{M}_{0}^{(S \varphi)}}{\operatorname{Det} \widehat{\mathcal{M}}_{0}^{(S \varphi)}}=\left(\frac{\operatorname{det} \Psi_{0}^{(S \varphi)}\left(r_{0}\right)}{\operatorname{det} \widehat{\Psi}_{0}^{(S \varphi)}\left(r_{0}\right)}\right)^{-1}\left(\frac{\operatorname{det} \Psi_{0}^{(S \varphi)}\left(r_{\infty}\right)}{\operatorname{det} \widehat{\Psi}_{0}^{(S \varphi)}\left(r_{\infty}\right)}\right) .
$$

Importantly, however, extra treatment is needed when there exists an unbroken gauge symmetry at the false vacuum. If it exists, there shows up a gauge zero mode, which makes eq. (4.42) vanish. Indeed, it is easy to see the existence of zero modes explicitly. For $\ell=0$, the Type 1 solution given in eq. (4.33) has $n_{G}$ columns distinguished by the adjoint index of $M$. Because $M_{i a}(r \rightarrow \infty)=0$ if the adjoint index $a$ is for unbroken generators at the false vacuum, $\operatorname{det} \mathcal{M}_{0}^{(S \varphi)}$ vanishes if there exists an unbroken gauge symmetry at the false vacuum. In our setup, the number of gauge zero modes is $n_{U}$.

If there exist zero modes, a naive calculation of the prefactor $\mathcal{A}$ makes it divergent. Such a divergence is an artifact arising from the flat directions of the Euclidean action, implying the break down of the saddle point method in the path integral. The proper treatments of the zero modes will be discussed in section 5 . 


\subsection{Background gauge}

Although it is convenient to use the Fermi gauge to discuss the gauge invariance of the decay rate, the background gauge is useful in performing the numerical calculation of the decay rate. Here, we show that the calculations based on the Fermi and the background gauges give the same result for $\ell>0$. (For $\ell=0$, the zero mode subtraction is non-trivial in the background gauge. The treatments of the zero modes will be discussed in the next section.)

In order to discuss the functional determinants in the background gauge, we first define the function $\Psi_{\ell, \mathrm{BG}}^{(c \bar{c})}$ which is $n_{G} \times n_{G}$. It obeys the following differential equation:

$$
\left(-\Delta_{\ell}+\xi M^{T} M\right) \Psi_{\ell, \mathrm{BG}}^{(c \bar{c})}=0
$$

with the boundary condition

$$
\Psi_{\ell, \mathrm{BG}}^{(c \bar{c})}(r \rightarrow 0)=r^{\ell} I_{G}
$$

In our convention, because $M^{T} M(r \rightarrow \infty)$ reduces to the block-diagonal form (see eq. (2.28)), the relevant part of $\Psi_{\ell, \mathrm{BG}}^{(c \bar{c})}$ for our analysis behaves as

$$
\Psi_{\ell, \mathrm{BG}}^{(c \bar{c})}(r \rightarrow \infty) \simeq\left(\begin{array}{cc}
f_{\ell, B}^{(c \bar{c})} & 0 \\
0 & f_{\ell, U}^{(c \bar{c})}
\end{array}\right) \tau^{(c \bar{c})}
$$

where $f_{\ell, B}^{(c \bar{c})}$ and $f_{\ell, U}^{(c \bar{c})}$ are $n_{B} \times n_{B}$ and $n_{U} \times n_{U}$, respectively, while $\tau^{(c \bar{c})}$ is an $n_{G} \times n_{G}$ orthogonal matrix. Notice that $f_{\ell, B}^{(c \bar{c})}(r \rightarrow \infty)$ exponentially grows while $f_{\ell, U}^{(c \bar{c})}(r \rightarrow \infty)$ is approximately proportional to $r^{\ell}$.

Similarly, we also define the function $\widehat{\Psi}_{\ell, \mathrm{BG}}^{(c \bar{c})}$, obeying

$$
\left(-\Delta_{\ell}+\xi \widehat{M}^{T} \widehat{M}\right) \widehat{\Psi}_{\ell, \mathrm{BG}}^{(c \bar{c})}=0
$$

and

$$
\widehat{\Psi}_{\ell, \mathrm{BG}}^{(c \bar{c})}(r \rightarrow 0)=r^{\ell} I_{G}
$$

Because of the block-diagonal nature of $\widehat{M}^{T} \widehat{M}$ in our convention, $\widehat{\Psi}_{\ell, \mathrm{BG}}^{(c \bar{c})}$ can be expressed as

$$
\widehat{\Psi}_{\ell, \mathrm{BG}}^{(c \bar{c})}=\left(\begin{array}{cc}
\widehat{f}_{\ell, B}^{(c \bar{c})} & 0 \\
0 & \widehat{f}_{\ell, U}^{(c \bar{c})}
\end{array}\right)
$$

where $\widehat{f}_{\ell, B}^{(c \bar{c})}$ is an $n_{B} \times n_{B}$ object, while $\hat{f}_{\ell, U}^{(c \bar{c})}=r^{\ell} I_{U}$. The functional determinant of the fluctuation operator of the FP ghosts is then given by

$$
\frac{\operatorname{Det} \mathcal{M}_{\ell, \mathrm{BG}}^{(c \bar{c})}}{\operatorname{Det} \widehat{\mathcal{M}}_{\ell, \mathrm{BG}}^{(c \bar{c})}}=\frac{\operatorname{det} \Psi_{\ell, \mathrm{BG}}^{(c \bar{c})}\left(r_{\infty}\right)}{\operatorname{det} \widehat{\Psi}_{\ell, \mathrm{BG}}^{(c \bar{c})}\left(r_{\infty}\right)} .
$$


For the calculation of the functional determinant of $\mathcal{M}_{\ell, \mathrm{BG}}^{(S L \varphi)}$, we should derive the solutions of the following differential equation:

$$
\mathcal{M}_{\ell, \mathrm{BG}}^{(S L \varphi)} \Psi_{\ell, \mathrm{BG}}^{(S L \varphi)}=0
$$

As in the case of the Fermi gauge, we can find a set of functions that are relevant for the determinant:

$$
\begin{aligned}
& \tilde{\psi}_{\mathrm{BG}}^{(1)}=(\begin{array}{cc}
\partial_{r} f_{\ell, B}^{(c \bar{c})} & 0 \\
0 & \ell f_{\ell, U}^{(c \bar{c})} \\
0 & 0 \\
0 & \frac{L}{r} f_{\ell, U}^{(c \bar{c})} \\
0 & 0 \\
0 & 0
\end{array} \underbrace{0}_{n_{B}}\}\left\{\begin{array}{l}
n_{B} \\
n_{U} \\
n_{B} \\
n_{U} \\
n_{B} \\
n_{\varphi}
\end{array}\right. \\
& \tilde{\psi}_{\mathrm{BG}}^{(2)}=\left(\begin{array}{cc}
0 & 0 \\
0 & \frac{\ell-(\ell+2) \xi}{4(\ell+2)} r f_{\ell, U}^{(c \bar{c})} \\
0 & 0 \\
0 & \frac{\ell[(\ell+4)-(\ell+2) \xi]}{4 L(\ell+2)} r f_{\ell, U}^{(c \bar{c})} \\
0
\end{array}\right\}\{\begin{array}{l}
n_{B} \\
n_{U} \\
n_{B}, \\
n_{U} \\
n_{n_{B}}
\end{array} \underbrace{}_{\varphi}
\end{aligned}
$$

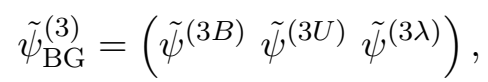

where terms irrelevant for our discussion are neglected. With these functions, $\Psi_{\ell, \mathrm{BG}}^{(S L \varphi)}(r \rightarrow \infty)$ can be expressed as

$$
\Psi_{\ell, \mathrm{BG}}^{(S L \varphi)}(r \rightarrow \infty)=\left(\tilde{\psi}_{\mathrm{BG}}^{(1)} \tilde{\psi}_{\mathrm{BG}}^{(2)} \tilde{\psi}_{\mathrm{BG}}^{(3)}\right)\left(\begin{array}{ccc}
\tau_{\mathrm{BG}}^{(c \bar{c})} & 0 & 0 \\
0 & \tau_{\mathrm{BG}}^{(c \bar{c}} & 0 \\
0 & 0 & I_{\varphi}
\end{array}\right)\left(\begin{array}{ccc}
I_{G} & 0 & 0 \\
0 & I_{G} & 0 \\
0 & 0 & \tau_{\mathrm{BG}}^{(\eta \lambda)}
\end{array}\right)+\text { (irrelevant) }
$$

with $\tau_{\mathrm{BG}}^{(\eta \lambda)}$ being an $n_{\varphi} \times n_{\varphi}$ orthogonal matrix.

Meanwhile, the behavior of $\Psi_{\ell, \mathrm{BG}}^{(S L \varphi)}$ around $r=0$ is given by

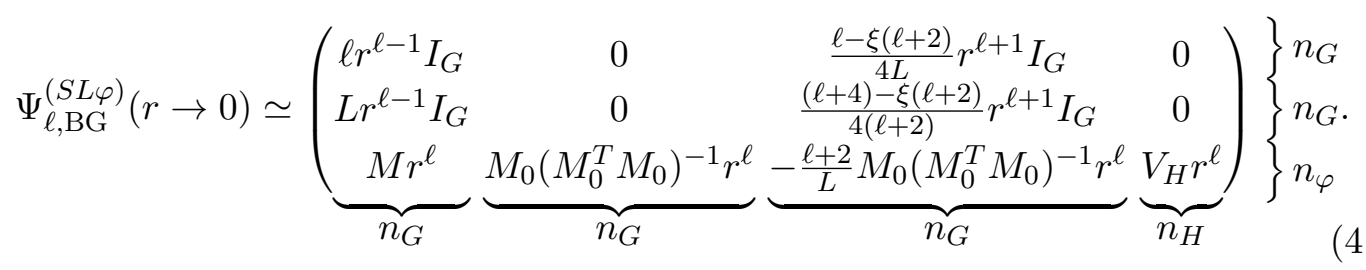


Asymptotic behavior of the function $\widehat{\Psi}_{\ell, \mathrm{BG}}^{(S L \varphi)}$, which obeys

$$
\widehat{\mathcal{M}}_{\ell, \mathrm{BG}}^{(S L \varphi)} \widehat{\Psi}_{\ell, \mathrm{BG}}^{(S L \varphi)}=0
$$

can be again obtained using the block-diagonalization given in eq. (2.52). We define $\widehat{\Psi}_{\ell, \mathrm{BG}}^{(X)}(X=B, U, \sigma)$ through $\widehat{\mathcal{M}}_{\ell, \mathrm{BG}}^{(X)} \widehat{\Psi}_{\ell, \mathrm{BG}}^{(X)}=0$, which describe independent solutions of the differential equation. For $\widehat{\Psi}_{\ell, \mathrm{BG}}^{(B)}$, we obtain

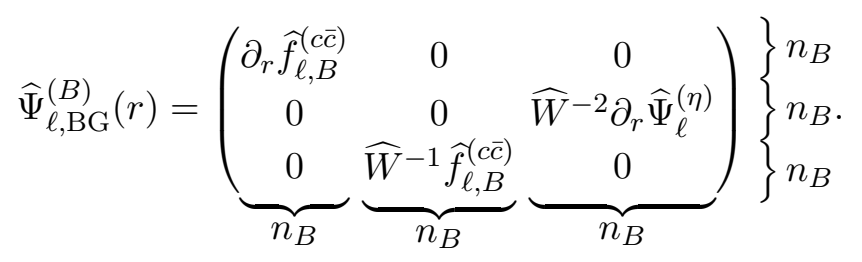

For the others, we can find $\widehat{\Psi}_{\ell, \mathrm{BG}}^{(U)}=\widehat{\Psi}_{\ell}^{(U)}$ and $\widehat{\Psi}_{\ell, \mathrm{BG}}^{(\sigma)}=\widehat{\Psi}_{\ell}^{(\sigma)}$. Combining all of them, $\operatorname{det} \widehat{\Psi}_{\ell, \mathrm{BG}}^{(S L \varphi)}(r)$ is given by

$$
\operatorname{det} \widehat{\Psi}_{\ell, \mathrm{BG}}^{(S L \varphi)}(r)=\operatorname{det}\left(\widehat{\Psi}_{\ell, \mathrm{BG}}^{(B)}(r)\right) \operatorname{det}\left(\widehat{\Psi}_{\ell, \mathrm{BG}}^{(U)}(r)\right) \operatorname{det}\left(\widehat{\Psi}_{\ell, \mathrm{BG}}^{(\sigma)}(r)\right)
$$

Finally, we can find

$$
\frac{\operatorname{det} \Psi_{\ell, \mathrm{BG}}^{(S L \varphi)}\left(r_{\infty}\right)}{\operatorname{det} \widehat{\Psi}_{\ell, \mathrm{BG}}^{(S L \varphi)}\left(r_{\infty}\right)}=\left(\frac{\operatorname{det} \Psi_{\ell \mathrm{BG}}^{(c \bar{c})}\left(r_{\infty}\right)}{\operatorname{det} \widehat{\Psi}_{\ell, \mathrm{BG}}^{(c \bar{c})}\left(r_{\infty}\right)}\right)^{2} \frac{\operatorname{det} \Psi_{\ell}^{(S L \varphi)}\left(r_{\infty}\right)}{\operatorname{det} \widehat{\Psi}_{\ell}^{(S L \varphi)}\left(r_{\infty}\right)}
$$

and consequently,

$$
\frac{\operatorname{Det} \mathcal{M}_{\ell, \mathrm{BG}}^{(S L \varphi)}}{\operatorname{Det} \widehat{\mathcal{M}}_{\ell, \mathrm{BG}}^{(S L \varphi)}}=\left(\frac{\operatorname{det} \Psi_{\ell, \mathrm{BG}}^{(c \bar{c})}\left(r_{\infty}\right)}{\operatorname{det} \widehat{\Psi}_{\ell, \mathrm{BG}}^{(c \bar{c})}\left(r_{\infty}\right)}\right)^{2} \frac{\operatorname{Det} \mathcal{M}_{\ell}^{(S L \varphi)}}{\operatorname{Det} \widehat{\mathcal{M}}_{\ell}^{(S L \varphi)}}
$$

In the Fermi and the background gauges, the functional determinants of the fluctuation operators of the $(S L \varphi)$ modes differ from each other. In the calculation of the prefactor $\mathcal{A}$, the difference is compensated by the contributions from the FP ghosts. Indeed, we can find

$$
\left(\frac{\operatorname{Det} \mathcal{M}_{\ell, \mathrm{BG}}^{(S L \varphi)}}{\operatorname{Det} \widehat{\mathcal{M}}_{\ell, \mathrm{BG}}^{(S L \varphi)}}\right)^{-1 / 2} \frac{\operatorname{Det} \mathcal{M}_{\ell, \mathrm{BG}}^{(c \bar{c})}}{\operatorname{Det} \widehat{\mathcal{M}}_{\ell, \mathrm{BG}}^{(c \bar{c})}}=\left(\frac{\operatorname{Det} \mathcal{M}_{\ell}^{(S L \varphi)}}{\operatorname{Det} \widehat{\mathcal{M}}_{\ell}^{(S L \varphi)}}\right)^{-1 / 2} \frac{\operatorname{Det} \mathcal{M}_{\ell}^{(c \bar{c})}}{\operatorname{Det} \widehat{\mathcal{M}}_{\ell}^{(c \bar{c})}}
$$

The above relation guarantees the equivalence of the total functional determinants (on which the prefactor $\mathcal{A}$ depends) in the Fermi and the background gauges for $\ell \geq 1$. 
In performing the calculation in the background gauge, we should also study the $\ell=0$ modes. The boundary condition for $\ell=0$ (for the study of $\mathcal{M}_{0, \mathrm{BG}}^{(S \varphi)}$ ) are also obtained as

$$
\begin{aligned}
& \Psi_{0, \mathrm{BG}}^{(S \varphi)(1)}(r \rightarrow 0) \simeq(\underbrace{\left.\begin{array}{c}
\frac{\xi}{4} r\left(M_{0}^{T} M_{0}\right) \\
M_{0}
\end{array}\right)}_{n_{G}}\} n_{G},
\end{aligned}
$$

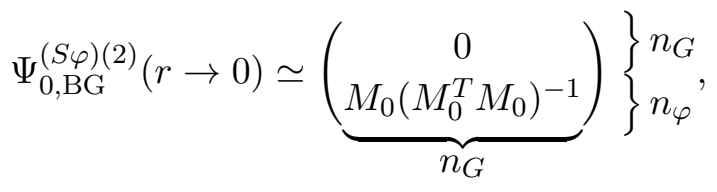

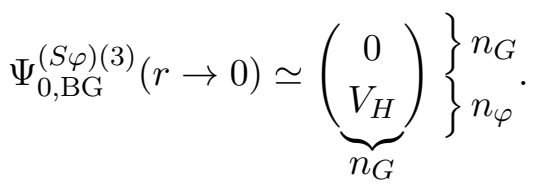

For $\ell=0$, special care is necessary when there exist gauge zero modes. In such a case, both Det $\mathcal{M}_{0}^{(S \varphi)}$ and Det $\mathcal{M}_{0, \mathrm{BG}}^{(S \varphi)}$ vanish and the comparison between the calculations in the Fermi and the background gauges is non-trivial (see the discussion in the next section).

\section{Zero modes}

When the action has flat directions around the bounce, mode functions corresponding to those directions become zero modes of fluctuation operators. Here, we consider the zero modes in association with continuous symmetries of the theory. In particular, in the case of our interest, the translational and the gauge symmetries result in zero modes and their proper treatments are essential to calculate the prefactor, $\mathcal{A}$. In this section, we discuss how we can deal with these zero modes.

\subsection{General issues}

Let us first discuss the treatment of zero modes in general. As we have mentioned in the previous section, the ratio of the functional determinants of $n \times n$ fluctuation operators, $\mathcal{M}$ and $\widehat{\mathcal{M}},{ }^{9}$ is evaluated as

$$
\frac{\operatorname{Det} \mathcal{M}}{\operatorname{Det} \widehat{\mathcal{M}}}=\left(\frac{\operatorname{det} \Psi\left(r_{0}\right)}{\operatorname{det} \widehat{\Psi}\left(r_{0}\right)}\right)^{-1}\left(\frac{\operatorname{det} \Psi\left(r_{\infty}\right)}{\operatorname{det} \widehat{\Psi}\left(r_{\infty}\right)}\right),
$$

where $\Psi(r)$ and $\widehat{\Psi}(r)$ satisfy $\mathcal{M} \Psi=0$ and $\widehat{\mathcal{M}} \widehat{\Psi}=0$, respectively. Notice that $\Psi$ and $\widehat{\Psi}$ are $n \times n$ matrices, whose columns are linearly independent solutions, i.e.,

$$
\begin{aligned}
\Psi(r) & =\left(\psi^{(1)}(r) \cdots \psi^{(n)}(r)\right), \\
\widehat{\Psi}(r) & =\left(\widehat{\psi}^{(1)}(r) \cdots \widehat{\psi}^{(n)}(r)\right),
\end{aligned}
$$

\footnotetext{
${ }^{9}$ Here, we omit the superscripts and the subscript for notational simplicity.
} 
with $\mathcal{M} \psi^{(I)}=0$ and $\widehat{\mathcal{M}} \widehat{\psi}^{(I)}=0$. When $\mathcal{M}$ has zero eigenvalues, $\operatorname{det} \Psi\left(r_{\infty}\right)=0$ and some of $\psi^{(I)}\left(r_{\infty}\right)$ 's are not independent. Here, the number of the dependent columns of $\Psi\left(r_{\infty}\right)$ matches that of the zero modes, which we denote as $n_{\text {zero }}$. In this subsection, we take a basis in which

$$
\psi^{\left(I \leq n_{\text {zero }}\right)}\left(r_{\infty}\right)=0, \psi^{\left(I>n_{\text {zero }}\right)}\left(r_{\infty}\right) \neq 0 .
$$

(In the following subsections, we may use a different convention.)

The functional determinant is due to the use of the saddle point method in the path integral. To evaluate the path integral, we first expand the fields around the bounce configuration. Let us denote the fields as $\Phi$; in the case of our interest, $\Phi$ contains the gauge and scalar fields, i.e., $\Phi \ni\left(A_{\mu}, \phi\right)$. Then, we expand $\Phi$ around the bounce configuration (which is denoted as $\bar{\Phi}$ ) as

$$
\Phi=\bar{\Phi}+\sum_{a} c_{a} \mathcal{G}_{a}(x)
$$

where $c_{a}$ 's are expansion coefficients and $\mathcal{G}_{a}(x)$ 's denote the eigenfunctions of the fluctuation operator:

$$
\mathcal{M G}_{a}(x)=\omega_{a} \mathcal{G}_{a}(x)
$$

with $\omega_{a}$ 's being the corresponding eigenvalues. The eigenfunctions should satisfy $\mathcal{G}_{a}\left(r_{\infty}\right)=0$, and are normalized as ${ }^{10}$

$$
\left\langle\mathcal{G}_{a} \mid \mathcal{G}_{b}\right\rangle=\delta_{a b} .
$$

Then, the path integral is evaluated as

$$
\int \mathcal{D} \Phi e^{-S[\Phi]} \simeq e^{-S[\bar{\Phi}]} \int\left(\prod_{a} \frac{d c_{a}}{\sqrt{2 \pi}} e^{-\frac{1}{2} \omega_{a} c_{a}^{2}}\right) .
$$

By performing the Gaussian integrals, we obtain

$$
\int \mathcal{D} \Phi e^{-S[\Phi]} \simeq e^{-S[\bar{\Phi}]}\left(\prod_{a} \omega_{a}\right)^{-1 / 2} \equiv e^{-S[\bar{\Phi}]}(\operatorname{Det} \mathcal{M})^{-1 / 2}
$$

If there exist zero modes, some of $\omega_{a}$ 's vanish and the above result diverges. In such a case, we cannot use the naive saddle point method to evaluate the path integral.

When the zero mode is related to the symmetry of the theory, we may properly eliminate the zero modes and avoid the divergence of the transition amplitude mentioned above, as discussed in [28] for the case of translational zero modes. Let us denote a generic symmetry transformation of the bounce configuration as

$$
\bar{\Phi} \rightarrow \bar{\Phi}+\sum_{A} z_{A} \mathcal{F}_{A}(x)+\mathcal{O}\left(z^{2}\right)
$$

\footnotetext{
${ }^{10}$ The inner product is defined as
}

$$
\left\langle\mathcal{G}_{a} \mid \mathcal{G}_{b}\right\rangle=\int d^{4} x \mathcal{G}_{a} \cdot \mathcal{G}_{b}
$$


where $z_{A}$ denotes the transformation parameter. With the symmetry transformation, the bounce action is invariant so that $\mathcal{F}_{A}$ satisfies

$$
\mathcal{M F}_{A}=0
$$

with

$$
\lim _{r \rightarrow \infty} \mathcal{F}_{A}(x)=0
$$

Thus, the following relation holds

$$
\sum_{a \in \mathcal{I}_{0}} c_{a} \mathcal{G}_{a}(x)=\sum_{A} z_{A} \mathcal{F}_{A}(x),
$$

where $\mathcal{I}_{0}$ denotes the set of indices for the zero modes, resulting in

$$
c_{a}=\sum_{A}\left\langle\mathcal{G}_{a} \mid \mathcal{F}_{\mathcal{A}}\right\rangle z_{A}: \quad a \in \mathcal{I}_{0}
$$

Using

$$
\left\langle\mathcal{F}_{A} \mid \mathcal{F}_{B}\right\rangle=\sum_{a \in \mathcal{I}_{0}}\left\langle\mathcal{F}_{A} \mid \mathcal{G}_{a}\right\rangle\left\langle\mathcal{G}_{a} \mid \mathcal{F}_{B}\right\rangle
$$

the Jacobian to convert the variable $c_{a}$ to $z_{A}$ is found to be

$$
\mathcal{J}=\sqrt{\operatorname{det}_{A B}\left\langle\mathcal{F}_{A} \mid \mathcal{F}_{B}\right\rangle}
$$

where $\operatorname{det}_{A B}$ denotes the determinant of the matrix with the indices $A$ and $B$.

Based on the above argument, we reinterpret the path integral containing zero modes related to the symmetry as

$$
\int \mathcal{D} \Phi e^{-S[\Phi]} \rightarrow e^{-S[\bar{\Phi}]} \int\left(\prod_{A} \frac{d z_{A}}{\sqrt{2 \pi}}\right) \mathcal{J}\left(\operatorname{Det}^{\prime} \mathcal{M}\right)^{-1 / 2},
$$

where $\operatorname{Det}^{\prime} \mathcal{M}$ denotes the minor determinant of $\mathcal{M}$ with its zero eigenvalues eliminated:

$$
\operatorname{Det}^{\prime} \mathcal{M} \equiv \prod_{a \notin \mathcal{I}_{0}} \omega_{a}
$$

With properly interpreting the integrals over $z_{A}$ 's, the divergence originating from the zero modes may be avoided.

The minor determinant, $\operatorname{Det}^{\prime} \mathcal{M}$, can be calculated by regulating the fluctuation operator as

$$
\mathcal{M}_{\text {reg }} \equiv \mathcal{M}+\nu
$$

where $\nu$ is a constant. With $\nu$ being small enough, non-zero eigenvalues of $\mathcal{M}$ are (almost) unchanged while the zero eigenvalues are lifted by $\nu$. Consequently,

$$
\operatorname{Det}^{\prime} \mathcal{M}=\lim _{\nu \rightarrow 0} \frac{1}{\nu^{n_{\text {zero }}}} \operatorname{Det} \mathcal{M}_{\text {reg }} .
$$


We can calculate Det $\mathcal{M}_{\text {reg }}$ by using the procedure mentioned above. We can obtain a solution of $\mathcal{M}_{\text {reg }} \Psi_{\text {reg }}=0$, where $\Psi_{\text {reg }}$ is $n \times n$ and has $n$ linearly independent columns:

$$
\Psi_{\mathrm{reg}}(r)=\left(\psi_{\mathrm{reg}}^{(1)}(r) \cdots \psi_{\mathrm{reg}}^{(n)}(r)\right)
$$

Then, Det $\mathcal{M}_{\text {reg }}$ can be obtained by using $\Psi_{\text {reg }}$.

Because our purpose is to evaluate $\operatorname{det} \Psi_{\text {reg }}\left(r_{\infty}\right)$ up to $O\left(\nu^{n_{\text {zero }}}\right)$, we only need to calculate the columns in association with the zero eigenvalues up to $O(\nu)$; for the other columns, we can take $\psi_{\text {reg }}^{(I)} \simeq \psi^{(I)}\left(I>n_{\text {zero }}\right)$. We can calculate $\psi_{\text {reg }}^{(I)}$ related to the zero modes by treating $\nu$ as a perturbation. We introduce the function $\check{\psi}^{(I)}$ as

$$
\psi_{\text {reg }}^{\left(I \leq n_{\text {zero }}\right)}=\psi^{\left(I \leq n_{\text {zero }}\right)}+\nu \check{\psi}^{\left(I \leq n_{\text {zero }}\right)}+O\left(\nu^{2}\right),
$$

where the superscript " $\left(I \leq n_{\text {zero }}\right)$ " indicates the columns in association with the zero modes. Then, $\check{\psi}^{\left(I \leq n_{\text {zero }}\right)}$ should satisfy

$$
\mathcal{M} \check{\psi}^{\left(I \leq n_{\text {zero }}\right)}=-\psi^{\left(I \leq n_{\text {zero }}\right)}
$$

with $\psi^{\left(I \leq n_{\text {zero }}\right)}\left(r_{0}\right)=0$. With $\check{\psi}^{\left(I \leq n_{\text {zero }}\right)}$ being obtained by solving the above equation, we can take care of the zero modes as

$$
\left[\frac{\operatorname{Det} \mathcal{M}}{\operatorname{Det} \widehat{\mathcal{M}}}\right]^{-1 / 2} \rightarrow \int\left(\prod_{A} \frac{d z_{A}}{\sqrt{2 \pi}}\right) \mathcal{J}\left[\frac{\operatorname{Det}^{\prime} \mathcal{M}}{\operatorname{Det} \widehat{\mathcal{M}}}\right]^{-1 / 2},
$$

where

$$
\frac{\operatorname{Det}^{\prime} \mathcal{M}}{\operatorname{Det} \widehat{\mathcal{M}}}=\left[\frac{\operatorname{det} \Psi\left(r_{0}\right)}{\operatorname{det} \widehat{\Psi}\left(r_{0}\right)}\right]^{-1} \lim _{\nu \rightarrow 0} \frac{1}{\nu^{n_{\text {zero }}}}\left[\frac{\operatorname{det}\left\{\Psi\left(r_{\infty}\right)+\nu \check{\Psi}\left(r_{\infty}\right)\right\}}{\operatorname{det} \widehat{\Psi}\left(r_{\infty}\right)}\right]
$$

with $\check{\Psi}$ being $n \times n$ function containing $\check{\psi}^{\left(I \leq n_{\text {zero }}\right)}$ :

$$
\check{\Psi} \equiv\left(\check{\psi}^{(1)} \cdots \check{\psi}^{\left(n_{\text {zero }}\right)} \quad 0 \cdots 0\right)
$$

Then, using the fact that

$$
\Psi\left(r_{\infty}\right)+\nu \check{\Psi}\left(r_{\infty}\right)=\left(\nu \check{\psi}^{(1)}\left(r_{\infty}\right) \cdots \nu \check{\psi}^{\left(n_{\text {zero }}\right)}\left(r_{\infty}\right) \psi^{\left(n_{\text {zero }}+1\right)}\left(r_{\infty}\right) \cdots \psi^{(n)}\left(r_{\infty}\right)\right),
$$

we obtain

$$
\frac{\operatorname{Det}^{\prime} \mathcal{M}}{\operatorname{Det} \widehat{\mathcal{M}}}=\left[\frac{\operatorname{det} \Psi\left(r_{0}\right)}{\operatorname{det} \widehat{\Psi}\left(r_{0}\right)}\right]^{-1}\left[\frac{\operatorname{det}\left\{\Psi\left(r_{\infty}\right)+\check{\Psi}\left(r_{\infty}\right)\right\}}{\operatorname{det} \widehat{\Psi}\left(r_{\infty}\right)}\right] .
$$

The actual calculations of $\operatorname{Det}^{\prime} \mathcal{M}$ for the gauge and translational zero modes will be discussed in the following subsections. 


\subsection{Gauge zero modes}

We first consider the gauge zero modes. As we have mentioned in the previous section, if a gauge symmetry, which is broken by the bounce, is restored at the false vacuum, there show up zero modes in $\ell=0$ fluctuation operators. In this subsection, we apply the discussion in the previous subsection to the gauge zero modes.

The gauge zero modes can be given in the following form (see eq. (4.33)):

$$
\psi^{(A)}=\left(\begin{array}{c}
0 \\
M
\end{array}\right) \mathcal{U}^{(A)},
$$

where $\mathcal{U}^{(A)}$ 's are defined as

$$
\left(\mathcal{U}^{(1)} \cdots \mathcal{U}^{\left(n_{U}\right)}\right) \equiv\left(\begin{array}{c}
0 \\
I_{U}
\end{array}\right)\left\{\begin{array}{l}
n_{B} \\
n_{U}
\end{array}\right.
$$

Notice that $\psi^{(A)}$ is a column of $\Psi_{0}^{(S \varphi)(1)}$ and hence satisfy $\mathcal{M}_{0}^{(S \varphi)} \psi^{(A)}=0$. Since the last $n_{U}$ columns of $M$ vanish at $r \rightarrow r_{\infty}, \psi^{(A)}\left(r_{\infty}\right)=0$ and hence $\psi^{(A)}$ are eigenfunctions of $\mathcal{M}_{0}^{(S \varphi)}$ with zero eigenvalues.

In order to follow the prescription given in the previous subsection, we first calculate the function $\check{\psi}^{(A)}$ that satisfies

$$
\mathcal{M}_{0}^{(S \varphi)} \check{\psi}^{(A)}=-\psi^{(A)},
$$

with the boundary condition of

$$
\check{\psi}^{(A)}\left(r_{0}\right)=0 .
$$

The function $\check{\psi}^{(A)}$ can be decomposed as

$$
\check{\psi}^{(A)}=\left(\begin{array}{c}
\partial_{r} \check{\chi}^{(A)} \\
M \check{\chi}^{(A)}
\end{array}\right)+\left(\begin{array}{c}
-2\left(M^{T} M\right)^{-1}\left(M^{\prime}\right)^{T} \check{\lambda}^{(A)} \\
\check{\lambda}^{(A)}
\end{array}\right)+\left(\begin{array}{c}
{\left[\partial_{r}\left(M^{T} M\right)^{-1}\right] \check{\zeta}^{(A)}} \\
M\left(M^{T} M\right)^{-1} \check{\zeta}^{(A)}
\end{array}\right),
$$

where $\check{\chi}^{(A)}, \check{\zeta}^{(A)}$, and $\check{\lambda}^{(A)}$ evolve as

$$
\begin{aligned}
& \Delta_{0} \check{\chi}^{(A)}=\left.\Delta_{0} \chi\right|_{\chi \rightarrow \check{\chi}^{(A)}, \zeta \rightarrow \check{\zeta}^{(A)}, \lambda \rightarrow \check{\lambda}^{(A)},}, \\
& \Delta_{0} \check{\zeta}^{(A)}=M^{T} M \mathcal{U}^{(A)}, \\
& \Delta_{0} \check{\lambda}^{(A)}=\left.\Delta_{0} \lambda\right|_{\chi \rightarrow \check{\chi}^{(A)}, \zeta \rightarrow \check{\zeta}^{(A)}, \lambda \rightarrow \check{\lambda}^{(A)},},
\end{aligned}
$$

and $\check{\lambda}$ satisfies

$$
M^{T} \check{\lambda}=0 .
$$

One can solve eq. (5.35) to obtain

$$
\check{\zeta}^{(A)}(r)=\int_{0}^{r} d r_{1} r_{1}^{-3} \int_{0}^{r_{1}} d r_{2} r_{2}^{3} M^{T} M\left(r_{2}\right) \mathcal{U}^{(A)}+\text { (irrelevant). }
$$


As $r$ becomes large, $\check{\zeta}^{(A)}(r)$ becomes constant because $M^{T} M \mathcal{U}^{(A)}$ approaches to zero exponentially. Then, the relevant part of $\check{\psi}^{(A)}$ for the calculation of the prefactor $\mathcal{A}$ is given by

$$
\check{\psi}^{(A)}(r)=\left(\begin{array}{c}
0 \\
M_{U} \mathcal{I}_{U} \mathcal{X}_{U} \mathcal{U}^{(A)}
\end{array}\right)
$$

where $\mathcal{I}_{U}$ and $\mathcal{X}_{U}^{(A, B)}$ are $n_{U} \times n_{U}$ objects whose $(A, B)$ elements are given by

$$
\mathcal{I}_{U}^{(A, B)}(r) \equiv \int^{r} d r r^{-3} \mathcal{U}^{(A) T}\left(M^{T} M\right)^{-1} \mathcal{U}^{(B)},
$$

and

$$
\mathcal{X}_{U}^{(A, B)} \equiv \frac{1}{2 \pi^{2}} \int d^{4} x \mathcal{U}^{(A) T} M^{T} M \mathcal{U}^{(B)}
$$

Thus, because

$$
\left.\operatorname{Det}^{\prime} \mathcal{M}_{0}^{(S \varphi)} \propto \operatorname{det}\left(\begin{array}{cccc}
0 & 0 & -\frac{1}{4} \xi r I_{G} & 0 \\
M_{B} & M_{U} \mathcal{I}_{U} \mathcal{X}_{U} & 0 & \tilde{\lambda}
\end{array}\right)\right|_{r \rightarrow \infty}
$$

with $\tilde{\lambda}$ being the function introduced in eq. (4.35), we can find

$$
\frac{\operatorname{Det}^{\prime} \mathcal{M}_{0}^{(S \varphi)}}{\operatorname{Det} \widehat{\mathcal{M}}_{0}^{(S \varphi)}}=\operatorname{det}_{A B} \mathcal{X}_{U}^{(A, B)} \operatorname{det}_{A B} \mathcal{I}_{U}^{(A, B)}\left[\frac{\operatorname{det} \widehat{\Psi}_{0}^{(\sigma)}\left(r_{\infty}\right)}{\operatorname{det} \widehat{\Psi}_{0}^{(\sigma)}\left(r_{0}\right)}\right]^{-1}\left[\frac{\operatorname{det} \Psi_{0}^{(\lambda)}\left(r_{\infty}\right)}{\operatorname{det} \Psi_{0}^{(\lambda)}\left(r_{0}\right)}\right]\left[\frac{\operatorname{det}\left(M^{T} M\right)_{\infty}}{\operatorname{det}\left(M_{0}^{T} M_{0}\right)}\right]^{1 / 2},
$$

with

$$
\Psi_{0}^{(\lambda)} \equiv V_{H}^{T} \tilde{\lambda}
$$

The gauge zero modes are associated with the gauge symmetries that are restored at the false vacuum; the NG bosons in association with the global gauge transformations are not eaten by the gauge bosons and appear as the zero modes. Thus, the path integration over the gauge zero modes can be replaced by the integration over the gauge volume of the unbroken gauge symmetry at the false vacuum.

We parameterize such a global gauge transformation of the bounce configuration as

$$
\bar{\phi} \rightarrow \bar{\phi}+\sum_{A} \theta^{A} \widetilde{T}^{A} \bar{\phi}+\mathcal{O}\left(\theta^{2}\right)
$$

where $\widetilde{T}^{A}$ 's are generators of unbroken gauge symmetry at the false vacuum with $\widetilde{T}^{A} \bar{\phi}$ being required to be orthogonal, i.e.,

$$
\int d^{4} x\left(\widetilde{T}^{A} \bar{\phi}\right)^{T} \widetilde{T}^{B} \bar{\phi}=0 \quad(\text { for } A \neq B) .
$$

Here, the generators $\widetilde{T}^{A}$ are introduced to set a diagonal basis for the integration over the gauge volume, and are given by linear combinations of the generators of our original choice, i.e.,

$$
\widetilde{T}^{A}=\kappa_{A B} T^{B}
$$


Note that the ambiguity in $\kappa$ is absorbed into the normalization of the gauge volume $\mathcal{V}_{U}$ (see the following discussion).

Since the fluctuation operator in the Fermi gauge is invariant under the transformation given in eq. (5.45), the path integral over the gauge zero modes can be interpreted as

$$
\int \prod_{a} \frac{d c_{a}^{\text {(gauge) }}}{\sqrt{2 \pi}} \rightarrow \mathcal{J}^{\text {(gauge) }} \int \prod_{A} \frac{d \theta^{A}}{\sqrt{2 \pi}} \equiv \frac{\mathcal{J}^{\text {(gauge) }}}{(2 \pi)^{n_{U} / 2}} \mathcal{V}_{U}
$$

where $c_{a}^{\text {(gauge) }}$ s are the expansion coefficients in association with the gauge zero modes, $\mathcal{V}_{U}$ is the volume of the moduli space arising from the spontaneous symmetry breaking, and

$$
\mathcal{J}^{\text {(gauge) }} \equiv \operatorname{det} \kappa\left[\operatorname{det}_{A B} \int d^{4} x\left(T^{A} \bar{\phi}\right)^{T} T^{B} \bar{\phi}\right]^{1 / 2}=\left(\prod_{A} \frac{g_{A}^{2}}{2 \pi^{2}}\right)^{-1 / 2} \operatorname{det} \kappa\left(\operatorname{det}_{A B} \mathcal{X}_{U}^{(A, B)}\right)^{1 / 2}
$$

Then, using $\operatorname{det} \widehat{\Psi}_{0}^{(\sigma)}\left(r_{0}\right)=\operatorname{det} \Psi_{0}^{(\lambda)}\left(r_{0}\right)=1$, the right-hand side of eq. (5.43) becomes

$$
\frac{\operatorname{Det}^{\prime} \mathcal{M}_{0}^{(S \varphi)}}{\operatorname{Det} \widehat{\mathcal{M}}_{0}^{(S \varphi)}}=\left(\frac{1}{2 r_{\infty}^{3}}\right)^{n_{U}}\left(\prod_{A} \frac{g_{A}^{2}}{2 \pi^{2}}\right)\left(\frac{\mathcal{J}^{\text {(gauge) }}}{\operatorname{det} \kappa}\right)^{2}\left(\operatorname{det} W_{U}^{\prime}\right)_{\infty}^{-1} \frac{\operatorname{det} \Psi_{0}^{(\lambda)}\left(r_{\infty}\right)}{\operatorname{det} \widehat{\Psi}_{0}^{(\sigma)}\left(r_{\infty}\right)}\left[\frac{\operatorname{det} \widehat{W}^{T} \widehat{W}}{\operatorname{det}\left(M_{0}^{T} M_{0}\right)}\right]^{1 / 2}
$$

and hence the $\ell=0$ contribution, containing the path integral over the gauge zero modes, can be written as

$$
\begin{aligned}
\int \prod_{a} \frac{d c_{a}^{\text {(gauge) }}}{\sqrt{2 \pi}}\left(\frac{\operatorname{Det}^{\prime} \mathcal{M}_{0}^{(S \varphi)}}{\operatorname{Det} \widehat{\mathcal{M}}_{0}^{(S \varphi)}}\right)^{-1 / 2} \rightarrow & \left(\frac{r_{\infty}^{3}}{\pi}\right)^{n_{U} / 2}\left(\prod_{A} \frac{g_{A}^{2}}{2 \pi^{2}}\right)^{-1 / 2} \mathcal{V}_{U} \operatorname{det} \kappa \\
& \left(\operatorname{det} W_{U}^{\prime}\right)^{1 / 2}\left[\frac{\operatorname{det} \Psi_{0}^{(\lambda)}\left(r_{\infty}\right)}{\operatorname{det} \widehat{\Psi}_{0}^{(\sigma)}\left(r_{\infty}\right)}\right]^{-1 / 2}\left[\frac{\operatorname{det} \widehat{W}^{T} \widehat{W}}{\operatorname{det}\left(M_{0}^{T} M_{0}\right)}\right]^{-1 / 4}
\end{aligned}
$$

Next, we consider the background gauge. The fluctuation operator in the background gauge, i.e., $\mathcal{M}_{0, \mathrm{BG}}^{(S \varphi)}$ given in eq. (2.49), also has zero eigenvalues in association with the unbroken gauge symmetry in the false vacuum. The existence of the zero eigenvalues of $\mathcal{M}_{0, \mathrm{BG}}^{(S \varphi)}$ can be understood from the fact that the following relation holds:

$$
\mathcal{M}_{0, \mathrm{BG}}^{(S \varphi)}\left(\begin{array}{l}
\partial_{r} \Psi_{0}^{(c \bar{c})} \\
M \Psi_{0}^{(c \bar{c})}
\end{array}\right)=0
$$

and that some of the columns of $\Psi_{0}^{(c \bar{c})}$ become constant at $r \rightarrow \infty$ if there exists an unbroken gauge symmetry at the false vacuum (see eq. (4.43)). The proper treatment of such zero modes is, however, complicated because the relation between the path integral over such zero modes and the integration over the gauge parameter is non-trivial in the background gauge. Here, we consider a way to reconstruct the result in the Fermi gauge from that in the background gauge. Even though the study in the Fermi gauge is enough for 
the gauge invariant formulation of the decay rate, the background gauge is advantageous for numerically calculating the decay rate. This is because the numerical calculation in the background gauge shows a better convergence at $r \rightarrow \infty$. Thus, it is desirable to understand the procedure to transform the results in the background gauge to those in the Fermi gauge. We will explicitly show such a transformation in the following.

In discussing the treatment of the gauge zero modes in the background gauge, we first define

$$
\begin{aligned}
& \mathcal{M}_{0, \mathrm{BG}, \mathrm{reg}}^{(S \varphi)}=\mathcal{M}_{0, \mathrm{BG}}^{(S \varphi)}+\nu\left(\begin{array}{cc}
\xi^{-1} I_{G} & 0 \\
0 & I_{\varphi}
\end{array}\right) \\
& \widehat{\mathcal{M}}_{0, \mathrm{BG}, \mathrm{reg}}^{(S \varphi)}=\widehat{\mathcal{M}}_{0, \mathrm{BG}}^{(S \varphi)}+\nu\left(\begin{array}{cc}
\xi^{-1} I_{G} & 0 \\
0 & I_{\varphi}
\end{array}\right) .
\end{aligned}
$$

Here, the regulator is chosen so that the final result becomes simple. Then, we can find (see appendix A)

$$
\frac{\operatorname{Det}^{\prime} \mathcal{M}_{0}^{(S \varphi)}}{\operatorname{Det} \widehat{\mathcal{M}}_{0}^{(S \varphi)}}=\frac{1}{\operatorname{det}_{A B} \mathcal{K}_{A B}}\left(\prod_{A} \frac{g_{A}^{2}}{2 \pi^{2}}\right)\left(\frac{\mathcal{J}^{\text {(gauge })}}{\operatorname{det} \kappa}\right)^{2}\left(\frac{\operatorname{Det} \mathcal{M}_{0, \mathrm{BG}}^{(c \bar{c})}}{\operatorname{Det} \widehat{\mathcal{M}}_{0, \mathrm{BG}}^{(c \bar{c})}}\right)^{-2} \lim _{\nu \rightarrow 0} \frac{1}{\nu^{n_{U}}} \frac{\operatorname{Det} \mathcal{M}_{0, \mathrm{BG}, \mathrm{reg}}^{(S \varphi)}}{\operatorname{Det} \widehat{\mathcal{M}}_{0, \mathrm{BG}, \mathrm{reg}}^{(S \varphi)}}
$$

where

$$
\mathcal{K}_{A B}=\lim _{r \rightarrow \infty} \frac{r^{3}}{\xi} \mathcal{U}^{(A) T}\left(\partial_{r} \Psi_{0}^{(c \bar{c})}\right)\left(\Psi_{0}^{(c \bar{c})}\right)^{-1} \mathcal{U}^{(B)} .
$$

In numerical calculations, eq. (5.55) can be used to obtain the Fermi gauge result from the calculation with the fluctuation operators in the background gauge. Here, one should note that, even though our regulator for the background gauge becomes equal to that of eq. (5.19) when $\xi=1$,

$$
\left.\left(\frac{\operatorname{Det}^{\prime} \mathcal{M}_{0, \mathrm{BG}}^{(S \varphi)}}{\operatorname{\operatorname {Det}} \widehat{\mathcal{M}}_{0, \mathrm{BG}}^{(S \varphi)}}\right)^{-1 / 2} \frac{\operatorname{Det} \mathcal{M}_{0, \mathrm{BG}}^{(c \bar{c})}}{\operatorname{Det} \widehat{\mathcal{M}}_{0, \mathrm{BG}}^{(c \bar{c})}}\right|_{\xi=1} \neq\left(\frac{\operatorname{Det}^{\prime} \mathcal{M}_{0}^{(S \varphi)}}{\operatorname{Det} \widehat{\mathcal{M}}_{0}^{(S \varphi)}}\right)^{-1 / 2} \frac{\operatorname{Det} \mathcal{M}_{0}^{(c \bar{c})}}{\operatorname{Det} \widehat{\mathcal{M}}_{0}^{(c \bar{c})}}
$$

The above inequality is not surprising because the gauge transformations relating different bounce configurations differ in two choices of the gauge fixing. In particular, in the background gauge, different bounces are related by a local, not global, gauge transformation [34]. Thus, if we worked only in the background gauge, it would become very non-trivial to relate the path integral over the zero modes to the integration over the gauge volume.

\subsection{Translational zero modes}

Next, let us consider the zero modes due to the translational invariance. These zero modes are related to

$$
\psi^{(\operatorname{tr})} \equiv \frac{r_{0}}{\sqrt{\bar{\phi}^{T}\left(r_{0}\right) \bar{\phi}^{\prime}\left(r_{0}\right)}}\left(\begin{array}{c}
0 \\
0 \\
\bar{\phi}^{\prime}
\end{array}\right)
$$


One can see that $\psi^{(\operatorname{tr})}$ satisfies $\mathcal{M}_{1}^{(S L \varphi)} \psi^{(\operatorname{tr})}=0$ and $\psi^{(\operatorname{tr})}(r \rightarrow \infty)=0$. We note here that $\psi^{(\operatorname{tr})}$ belongs to the Type 3 solutions (see eq. (4.9)). It corresponds to the solution obtained with $\chi^{(\operatorname{tr})}=\eta^{(\operatorname{tr})}=0$ and $\lambda^{(\operatorname{tr})} \propto \bar{\phi}^{\prime}$; the relation $\left(\Delta_{1}-\Omega\right) \bar{\phi}^{\prime}=0$ holds and that $\lambda^{(\operatorname{tr})} \propto \bar{\phi}^{\prime}$ satisfies the constraint imposed on the function $\lambda$, i.e., $M^{T} \lambda^{(\operatorname{tr})}=0$ (see eq. (2.13)). The normalization of $\psi^{(\operatorname{tr})}$ is determined so that $\psi^{(\operatorname{tr})}$ can be embedded into $\Psi_{\ell}^{(S L \varphi)(3)}$ (see eq. (4.10)).

We need to eliminate the zero eigenvalues from $\operatorname{Det} \mathcal{M}_{1}^{(S L \varphi)}$. For the calculation of $\operatorname{Det}^{\prime} \mathcal{M}_{1}^{(S L \varphi)}$, we should obtain the function satisfying

$$
\mathcal{M}_{1}^{(S L \varphi)} \check{\psi}^{(\mathrm{tr})}=-\psi^{(\mathrm{tr})} .
$$

The solution of the above equation can be expressed as

$$
\check{\psi}^{(\operatorname{tr})}=\left(\begin{array}{c}
\partial_{r} \check{\chi}^{(\operatorname{tr})} \\
\frac{L}{r} \check{\chi}^{(\operatorname{tr})} \\
M \check{\chi}^{(\operatorname{tr})}
\end{array}\right)+\left(\begin{array}{c}
\left(M^{T} M\right)^{-1}\left[\frac{L}{r} \check{\eta}^{(\operatorname{tr})}-2\left(M^{\prime}\right)^{T} \check{\lambda}^{(\operatorname{tr})}\right] \\
\left(M^{T} M\right)^{-1} \frac{1}{r^{2}} \partial_{r} r^{2} \check{\eta}^{(\operatorname{tr})} \\
\check{\lambda}^{(\operatorname{tr})}
\end{array}\right),
$$

where $\check{\chi}^{(\operatorname{tr})}, \check{\eta}^{(\operatorname{tr})}$, and $\check{\lambda}^{(\mathrm{tr})}$ are functions satisfying

$$
\begin{aligned}
& \Delta_{1} \check{\chi}^{(\operatorname{tr})}=\left.\Delta_{1} \chi\right|_{\chi \rightarrow \check{\chi}^{(\mathrm{tr})}, \eta \rightarrow \check{\eta}^{(\mathrm{tr})}, \lambda \rightarrow \check{\lambda}^{(\mathrm{tr})}, \zeta \rightarrow 0}, \\
& \Delta_{1} \check{\eta}^{(\mathrm{tr})}=\left.\Delta_{1} \eta\right|_{\chi \rightarrow \check{\chi}^{(\mathrm{tr})}, \eta \rightarrow \check{\eta}^{(\mathrm{tr})}, \lambda \rightarrow \check{\lambda}^{(\mathrm{tr})}, \zeta \rightarrow 0}, \\
& \Delta_{1} \check{\check{\lambda}}^{(\mathrm{tr})}=\left.\Delta_{1} \lambda\right|_{\chi \rightarrow \check{\chi}^{(\mathrm{tr})}, \eta \rightarrow \check{\eta}^{(\mathrm{tr})}, \lambda \rightarrow \check{\lambda}^{(\mathrm{tr})}, \zeta \rightarrow 0}-\frac{r_{0}}{\sqrt{\bar{\phi}^{T}\left(r_{0}\right) \bar{\phi}^{\prime}\left(r_{0}\right)}} \bar{\phi}^{\prime} .
\end{aligned}
$$

By solving the above equations, $\check{\chi}^{(\operatorname{tr})}, \check{\eta}^{(\operatorname{tr})}$, and $\check{\lambda}^{(\operatorname{tr})}$ can be obtained. Then, we can evaluate $\check{\psi}^{(\operatorname{tr})}(r \rightarrow \infty)$, with which $\operatorname{Det}^{\prime} \mathcal{M}_{1}^{(S L \varphi)}$ can be calculated.

The bounce is localized in space-time, and the shift of the position of the bounce does not change the action. Thus, in four space-time dimensions, there are four translational zero modes which can be parameterized as

$$
\left(\begin{array}{c}
\bar{A}_{\mu} \\
\bar{\phi}(x)
\end{array}\right) \rightarrow\left(\begin{array}{c}
\bar{A}_{\mu} \\
\bar{\phi}(x)
\end{array}\right)+d x_{\mu}\left(\begin{array}{c}
0 \\
\partial_{\mu} \bar{\phi}
\end{array}\right)+\mathcal{O}\left(d x^{2}\right)
$$

or as

$$
\left(\begin{array}{c}
\bar{A}_{\mu} \\
\bar{\phi}(x)
\end{array}\right) \rightarrow\left(\begin{array}{c}
\bar{A}_{\mu} \\
\bar{\phi}(x)
\end{array}\right)+c_{\left(1, m_{A}, m_{B}\right)}^{(\operatorname{tr})}\left(\begin{array}{c}
0 \\
\mathcal{N}^{(\operatorname{tr})} \bar{\phi}^{\prime} \mathcal{Y}_{\left(1, m_{A}, m_{B}\right)}
\end{array}\right)+\mathcal{O}\left(c^{2}\right)
$$

with $\mathcal{N}^{(\operatorname{tr})}$ being the normalization constant:

$$
\mathcal{N}^{(\mathrm{tr})}=\frac{1}{\sqrt{\int d r r^{3} \bar{\phi}^{\prime 2}}} .
$$

The path integral over the translational zero modes can be understood as the integration over the position of the center of the bounce as [28]:

$$
\prod_{m_{A}, m_{B}} \frac{d c_{\left(1, m_{A}, m_{B}\right)}^{(\operatorname{tr})}}{\sqrt{2 \pi}} \rightarrow \frac{\mathcal{B}^{2}}{4 \pi^{2}} \prod_{\mu} d x_{\mu}
$$


Consequently, the functional determinant from the path integral is interpreted as

$$
\left[\frac{\operatorname{Det} \mathcal{M}_{1}^{(S L \varphi)}}{\operatorname{Det} \widehat{\mathcal{M}}_{1}^{(S L \varphi)}}\right]^{-1 / 2} \rightarrow \frac{\mathcal{B}^{2}}{4 \pi^{2}}\left[\frac{\operatorname{Det}^{\prime} \mathcal{M}_{1}^{(S L \varphi)}}{\operatorname{Det} \widehat{\mathcal{M}}_{1}^{(S L \varphi)}}\right]^{-1 / 2} \prod_{\mu} d x_{\mu} .
$$

Integration over the space-time volume will disappear from the expression of the decay rate per unit volume (i.e., the transition probability per unit time and unit volume). We note that, in the background gauge, we can obtain eq. (5.68) with the fluctuation operators in the Fermi gauge being replaced by those in the background gauge.

\section{Semi-analytic expression of the decay rate}

Now, we summarize the semi-analytic expression of the decay rate of the false vacuum. We are particularly interested in the expression of the prefactor $\mathcal{A}$ and its gauge invariance. The prefactor $\mathcal{A}$ can be given as a product of the contributions of various fluctuations as

$$
\mathcal{A}=\mathcal{A}^{(c \bar{c})} \mathcal{A}^{(T)} \mathcal{A}^{(S L \varphi)},
$$

where $\mathcal{A}^{(c \bar{c})}, \mathcal{A}^{(T)}$, and $\mathcal{A}^{(S L \varphi)}$ denote the contributions of the FP ghosts, transverse mode of the gauge fields, and other bosonic fluctuations, respectively. (If there exist fermions coupled to the bounce, their contributions should be also taken into account.) Furthermore, because of the spherical nature of the bounce configuration, each contribution can be decomposed into those with different $\left(\ell, m_{A}, m_{B}\right)$ :

$$
\mathcal{A}^{(X)}=\prod_{\ell}\left[\mathcal{A}_{\ell}^{(X)}\right]^{(\ell+1)^{2}},
$$

with $(X)=(c \bar{c}),(T)$, or $(S L \varphi)$. (Here, $\mathcal{A}_{0}^{(S L \varphi)}$ should be understood as $\mathcal{A}_{0}^{(S \varphi)}$.) For $\mathcal{A}^{(c \bar{c})}$ and $\mathcal{A}^{(S L \varphi)}, 0 \leq \ell<\infty$ and for $\mathcal{A}^{(T)}, 1 \leq \ell<\infty$. The final result $\mathcal{A}$ does not depend on the choice of the gauge fixing (as far as the gauge zero modes are irrelevant), although $\mathcal{A}^{(c \bar{c})}$ and $\mathcal{A}^{(S L \varphi)}$ in the Fermi and the background gauges differ from each other. In the following, we first summarize the results in the Fermi gauge and then discuss those in the background gauge.

\subsection{Contributions of FP ghosts and transverse modes}

First, we consider the contributions of the FP ghosts. In the Fermi gauge, the FP ghosts do not couple to the bounce, and hence the fluctuation operator of FP ghosts does not contain $\bar{\phi}$. Consequently,

$$
\mathcal{A}^{(c \bar{c})}=1
$$

For the contributions of the transverse modes of the gauge fields, we should solve

$$
\begin{aligned}
& \mathcal{M}_{\ell}^{(T)} \Psi_{\ell}^{(T)}=0, \\
& \widehat{\mathcal{M}}_{\ell}^{(T)} \widehat{\Psi}_{\ell}^{(T)}=0,
\end{aligned}
$$


where $\mathcal{M}_{\ell}^{(T)}$ and $\widehat{\mathcal{M}}_{\ell}^{(T)}$ are the fluctuation operators given in eqs. (2.46) and (2.51), respectively. In addition, $\Psi_{\ell}^{(T)}$ and $\widehat{\Psi}_{\ell}^{(T)}$ are $n_{G} \times n_{G}$ objects, and their boundary conditions are given by

$$
\begin{aligned}
& \Psi_{\ell}^{(T)}(r \rightarrow 0) \simeq r^{\ell} I_{G}, \\
& \widehat{\Psi}_{\ell}^{(T)}(r \rightarrow 0) \simeq r^{\ell} I_{G} .
\end{aligned}
$$

With these quantities,

$$
\frac{\operatorname{det} \mathcal{M}_{\ell}^{(T)}}{\operatorname{det} \widehat{\mathcal{M}}_{\ell}^{(T)}}=\frac{\operatorname{det} \Psi_{\ell}^{(T)}\left(r_{\infty}\right)}{\operatorname{det} \widehat{\Psi}_{\ell}^{(T)}\left(r_{\infty}\right)}
$$

Then,

$$
\mathcal{A}^{(T)}=\prod_{\ell=1}^{\infty}\left[\frac{\operatorname{det} \Psi_{\ell}^{(T)}\left(r_{\infty}\right)}{\operatorname{det} \widehat{\Psi}_{\ell}^{(T)}\left(r_{\infty}\right)}\right]^{-(\ell+1)^{2} / 2} .
$$

\subsection{Contributions of $(S L \varphi)$ modes}

Contributions of the $(S L \varphi)$ modes are complicated especially because of the zero modes. The prefactor originating from the $(S L \varphi)$ modes can be written as

$$
\mathcal{A}^{(S L \varphi)}=\mathcal{A}_{0}^{(S \varphi)} \prod_{\ell=1}^{\infty}\left[\mathcal{A}_{\ell}^{(S L \varphi)}\right]^{(\ell+1)^{2}} .
$$

For $\ell>1$, we do not expect zero modes. Based on eq. (4.32), we obtain

$$
\begin{aligned}
\mathcal{A}_{\ell>1}^{(S L \varphi)}= & \left(\frac{r_{\infty}}{\ell}\right)^{n_{U} / 2}\left[\operatorname{det}\left(M^{T} M\right)_{\infty} \operatorname{det}\left(M_{0}^{T} M_{0}\right)\right]^{-1 / 4}\left[\operatorname{det}\left(1 / W_{U}^{T} W_{U}\right)_{\infty}^{\prime}\right]^{-1 / 2}\left[\operatorname{det} \widehat{W}^{T} \widehat{W}\right]^{1 / 4} \\
& {\left[\operatorname{det} \Psi_{\ell}^{(\eta \lambda)}\left(r_{\infty}\right)\right]^{-1 / 2}\left[\operatorname{det} \widehat{\Psi}_{\ell}^{(\eta)}\left(r_{\infty}\right)\right]^{1 / 2}\left[\operatorname{det} \widehat{\Psi}_{\ell}^{(\sigma)}\left(r_{\infty}\right)\right]^{1 / 2} }
\end{aligned}
$$

where $\Psi_{\ell}^{(\eta \lambda)}$ is give by eq. (4.30), while $\widehat{\Psi}_{\ell}^{(\eta)}$ and $\widehat{\Psi}_{\ell}^{(\sigma)}$ are defined by eqs. (4.23) and (4.24) and eqs. (4.26) and (4.27), respectively.

For $\ell=1$, the translational zero modes exist. The zero eigenvalues from the translational zero modes are removed by replacing $\eta^{(\operatorname{tr})}$ and $\lambda^{(\operatorname{tr})}$ by $\check{\eta}^{(\operatorname{tr})}$ and $\check{\lambda}^{(\operatorname{tr})}$, respectively, in $\operatorname{det} \Psi_{1}^{(S L \varphi)}(r \rightarrow \infty)$, with taking account of the Jacobian (see eq. (5.68)). As a result, $\mathcal{A}_{1}^{(S L \varphi)}$ is obtained as

$$
\begin{aligned}
\mathcal{A}_{1}^{(S L \varphi)}= & \frac{\mathcal{B}^{2}}{4 \pi^{2}} r_{\infty}^{n_{U} / 2}\left[\operatorname{det}\left(M^{T} M\right)_{\infty} \operatorname{det}\left(M_{0}^{T} M_{0}\right)\right]^{-1 / 4}\left[\operatorname{det}\left(1 / W_{U}^{T} W_{U}\right)_{\infty}^{\prime}\right]^{-1 / 2}\left[\operatorname{det} \widehat{W}^{T} \widehat{W}\right]^{1 / 4} \\
& {\left[\operatorname{det}\left(\Psi_{1}^{(\eta \lambda)}\left(r_{\infty}\right)+\check{\Psi}_{1}^{(\eta \lambda)}\left(r_{\infty}\right)\right)\right]^{-1 / 2}\left[\operatorname{det} \widehat{\Psi}_{1}^{(\eta)}\left(r_{\infty}\right)\right]^{1 / 2}\left[\operatorname{det} \widehat{\Psi}_{1}^{(\sigma)}\left(r_{\infty}\right)\right]^{1 / 2}, }
\end{aligned}
$$

where

$$
\Psi_{1}^{(\eta \lambda)}+\check{\Psi}_{1}^{(\eta \lambda)}=\left.\Psi_{1}^{(\eta \lambda)}\right|_{\psi^{(\mathrm{tr})} \rightarrow \check{\psi}^{(\mathrm{tr})}}
$$


Here, the definitions of $\psi^{(\operatorname{tr})}$ and $\check{\psi}^{(\operatorname{tr})}$ are given in eqs. (5.58) and (5.59), respectively. Notice that the space-time integral in eq. (5.68) is eliminated in calculating the decay rate per unit volume.

For $\ell=0$, there may exist gauge zero modes. Based on eq. (5.51), we obtain

$$
\mathcal{A}_{0}^{(S \varphi)}=\left(\frac{r_{\infty}^{3}}{\pi}\right)^{n_{U} / 2}\left(\prod_{A} \frac{g_{A}^{2}}{2 \pi^{2}}\right)^{-1 / 2} \mathcal{V}_{U} \operatorname{det} \kappa\left(\operatorname{det} W_{U}^{\prime}\right)^{1 / 2}\left[\frac{\operatorname{det} \Psi_{0}^{(\lambda)}\left(r_{\infty}\right)}{\operatorname{det} \widehat{\Psi}_{0}^{(\sigma)}\left(r_{\infty}\right)}\right]^{-1 / 2}\left[\frac{\operatorname{det} \widehat{W}^{T} \widehat{W}}{\operatorname{det}\left(M_{0}^{T} M_{0}\right)}\right]^{-1 / 4},
$$

where $\mathcal{V}_{U}$ is the gauge volume and $\kappa$ is the matrix orthogonalizing $T^{A} \bar{\phi}$ (see eq. (5.47)). In addition, $\Psi_{0}^{(\lambda)}$ is given in eq. (5.44).

\subsection{Background gauge}

Here, we summarize the relation between the results in the Fermi gauge and in the background gauge. In the background gauge, the FP contribution becomes

$$
\mathcal{A}_{\mathrm{BG}}^{(c \bar{c})}=\prod_{\ell=0}^{\infty}\left[\mathcal{A}_{\ell, \mathrm{BG}}^{(c \bar{c})}\right]^{(\ell+1)^{2}}=\prod_{\ell=0}^{\infty}\left[\frac{\operatorname{det} \Psi_{\ell \mathrm{BG}}^{(c \bar{c})}}{\operatorname{det} \widehat{\Psi}_{\ell, \mathrm{BG}}^{(c \bar{c})}}\right]^{(\ell+1)^{2}} .
$$

The functions $\Psi_{\ell, \mathrm{BG}}^{(c \bar{c})}$ and $\widehat{\Psi}_{\ell, \mathrm{BG}}^{(c \bar{c})}$ are defined in eqs. (4.43) and (4.44) and eqs. (4.46) and (4.47), respectively.

Since the fluctuation operators for the $(T)$ modes are identical to those in the Fermi gauge,

$$
\mathcal{A}_{\ell, \mathrm{BG}}^{(T)}=\mathcal{A}_{\ell}^{(T)} .
$$

As we have seen in eq. (4.61), the following relation holds for $\ell>0$ :

$$
\mathcal{A}_{\ell, \mathrm{BG}}^{(S L \varphi)} \mathcal{A}_{\ell, \mathrm{BG}}^{(c \bar{c})}=\mathcal{A}_{\ell}^{(S L \varphi)} \mathcal{A}_{\ell}^{(c \bar{c})} .
$$

Thus, we can explicitly see that the Fermi and the background gauges give the same decay rate as far as the modes with $\ell>0$ are concerned.

For $\ell=0$, the proper treatment of the gauge zero modes in the background gauge is non-trivial, as we have explained in section 5. Thus, in the background gauge, we just introduce a convenient way of regulating the fluctuation operator with gauge zero modes and relate its functional determinant with that in the Fermi gauge. With the regulated fluctuation operators given in eqs. (5.53) and (5.54), we can obtain

$\mathcal{A}_{0}^{(S \varphi)}=\mathcal{V}_{U}\left(\operatorname{det}_{A B} \mathcal{K}_{A B}\right)^{1 / 2} \operatorname{det} \kappa\left(\prod_{A} \frac{g_{A}^{2}}{\pi}\right)^{-1 / 2}\left(\frac{\operatorname{Det} \mathcal{M}_{0, \mathrm{BG}}^{(c \bar{c})}}{\operatorname{Det} \widehat{\mathcal{M}}_{0, \mathrm{BG}}^{(\mathrm{c} \bar{c})}}\right)\left(\lim _{\nu \rightarrow 0} \frac{1}{\nu^{n_{U}}} \frac{\operatorname{Det} \mathcal{M}_{0, \mathrm{BG}, \mathrm{reg}}^{(S \varphi)}}{\operatorname{Det} \widehat{\mathcal{M}}_{0, \mathrm{BG}, \mathrm{reg}}^{(S \varphi)}}\right)^{-1 / 2}$,

with $\mathcal{K}_{A B}$ being given in eq. (5.56). The above equality is useful for numerical calculations: the right-hand side of eq. (6.18) is written only with the quantities in the background gauge and hence we can calculate them numerically. 


\section{Renormalization}

The functional determinants diverge once the contributions of all $\ell$ are taken into account, and the renormalization is necessary. Here, we discuss how the divergences are removed, adopting the $\overline{\mathrm{MS}}$ scheme.

In calculating the one-loop contribution to the decay rate of the false vacuum, we are interested in the ratio of the functional determinants in the following form:

$$
\mathcal{A}^{(X)}=\left(\frac{\operatorname{Det} \mathcal{M}^{(X)}}{\operatorname{Det} \widehat{\mathcal{M}}^{(X)}}\right)^{-1 / 2}=\prod_{\ell=0}^{\infty}\left(\frac{\operatorname{Det} \mathcal{M}_{\ell}^{(X)}}{\operatorname{Det} \widehat{\mathcal{M}}_{\ell}^{(X)}}\right)^{-(\ell+1)^{2} / 2},
$$

where $\mathcal{M}^{(X)}$ and $\widehat{\mathcal{M}}^{(X)}$ are fluctuation operators around the bounce and that around the false vacuum, respectively. Our procedure of the renormalization is as follows [1]:

(i) We first identify terms which contain the divergence in $\ln \mathcal{A}^{(X)}$ (which we call $\delta \ln \mathcal{A}_{\text {div }}^{(X)}$ ).

(ii) Next, we perform the $\overline{\mathrm{MS}}$ subtraction of $\delta \ln \mathcal{A}_{\mathrm{div}}^{(X)}$. The result is denoted as $\delta \ln \mathcal{A}_{\mathrm{div}, \overline{\mathrm{MS}}}^{(X)}$.

Then, the divergence is removed as [1]

$$
\ln \mathcal{A}^{(X)} \rightarrow \ln \mathcal{A}_{\mathrm{MS}}^{(X)} \equiv \ln \mathcal{A}^{(X)}-\delta \ln \mathcal{A}_{\mathrm{div}}^{(X)}+\delta \ln \mathcal{A}_{\mathrm{div}, \overline{\mathrm{MS}}}^{(X)} .
$$

Notice that, in the above expression, $\ln \mathcal{A}^{(X)}$ and $\delta \ln \mathcal{A}_{\text {div }}^{(X)}$ are both divergent, while $\ln \mathcal{A}^{(X)}-\delta \ln \mathcal{A}_{\mathrm{div}}^{(X)}$ and $\delta \ln \mathcal{A}_{\mathrm{div}, \overline{\mathrm{MS}}}^{(X)}$ are finite.

The divergent part of the functional determinant can be obtained by a diagrammatic consideration. Let us define

$$
\delta \mathcal{M} \equiv \mathcal{M}^{(X)}-\widehat{\mathcal{M}}^{(X)}
$$

and $\delta \mathcal{M}_{\ell} \equiv \mathcal{M}_{\ell}^{(X)}-\widehat{\mathcal{M}}_{\ell}^{(X)}$. Then, treating $\delta \mathcal{M}$ as a perturbation, $\ln \mathcal{A}^{(X)}$ can be expressed as

$$
\ln \mathcal{A}^{(X)}=-\sum_{I=1}^{\infty} s_{I}^{(X)}
$$

where $s_{I}^{(X)}$ indicates the contribution of diagrams with $I$ being the number of insertions of $\delta \mathcal{M}$.

Before proceeding further, we comment on the choice of the gauge fixing which affects the calculation of Det $\mathcal{M}_{\ell}^{(S L \varphi)}$. The calculation can be performed in any gauge; as we have discussed in section 4, the Fermi and the background gauge calculations give the same result of Det $\mathcal{M}_{\ell}^{(S L \varphi)}$ for $\ell \geq 1$ and hence the calculation of the divergent part is possible in either gauge. In practice, however, it is convenient to work in the background gauge because the number of diagrams necessary to calculate the divergent part is reduced. This is because, in the background gauge, $\delta \mathcal{M}$ does not contain derivative operators; thus, only $s_{1}^{(S L \varphi)}$ and $s_{2}^{(S L \varphi)}$ are divergent. (In the Fermi gauge, in general, $s_{I<4}^{(S L \varphi)}$ are divergent.) Thus, we adopt the background gauge to calculate the divergent part. In the following, we consider the case where only $s_{1}^{(X)}$ and $s_{2}^{(X)}$ are divergent while $s_{I \geq 3}^{(X)}$, s are finite. 
We have calculated the functional determinants of fluctuation operators by using the functions satisfying $\mathcal{M}_{\ell}^{(X)} \Psi_{\ell}^{(X)}=0$ and $\widehat{\mathcal{M}}_{\ell}^{(X)} \widehat{\Psi}_{\ell}^{(X)}=0$. We can express $\Psi_{\ell}^{(X)}$ in the following form:

$$
\Psi_{\ell}^{(X)}=\widehat{\Psi}_{\ell}^{(X)}+\sum_{I=1}^{\infty} \delta \Psi_{\ell, I}^{(X)}
$$

where the following equations are satisfied:

$$
\begin{aligned}
\widehat{\mathcal{M}}_{\ell}^{(X)} \delta \Psi_{\ell, 1}^{(X)} & =-\delta \mathcal{M}_{\ell} \widehat{\Psi}_{\ell}^{(X)} \\
\widehat{\mathcal{M}}_{\ell}^{(X)} \delta \Psi_{\ell, I+1}^{(X)} & =-\delta \mathcal{M}_{\ell} \delta \Psi_{\ell, I}^{(X)} \quad(I \geq 1),
\end{aligned}
$$

with $\delta \Psi_{\ell, I}^{(X)}(r \rightarrow 0) \simeq 0$. Notice that $\delta \Psi_{\ell, I}^{(X)}$ can be understood as the term which is of the order of $(\delta \mathcal{M})^{I}$. Then, $\ln \mathcal{A}^{(X)}$ becomes

$$
\ln \mathcal{A}^{(X)}=-\lim _{r \rightarrow \infty} \sum_{\ell=0}^{\infty} \frac{(\ell+1)^{2}}{2}\left[\operatorname{Tr} \ln \left(\widehat{\Psi}_{\ell}^{(X)}+\sum_{I=1}^{\infty} \delta \Psi_{\ell, I}^{(X)}\right)-\operatorname{Tr} \ln \widehat{\Psi}_{\ell}^{(X)}\right],
$$

from which we obtain

$$
\begin{aligned}
& s_{1}^{(X)}=\lim _{r \rightarrow \infty} \sum_{\ell=0}^{\infty} \frac{(\ell+1)^{2}}{2}\left[\operatorname{Tr}\left[\widehat{\Psi}_{\ell}^{(X)}\right]^{-1} \delta \Psi_{\ell, 1}^{(X)}\right] \\
& s_{2}^{(X)}=\lim _{r \rightarrow \infty} \sum_{\ell=0}^{\infty} \frac{(\ell+1)^{2}}{2}\left[\operatorname{Tr}\left[\widehat{\Psi}_{\ell}^{(X)}\right]^{-1} \delta \Psi_{\ell, 2}^{(X)}-\frac{1}{2} \operatorname{Tr}\left[\widehat{\Psi}_{\ell}^{(X)}\right]^{-1} \delta \Psi_{\ell, 1}^{(X)}\left[\widehat{\Psi}_{\ell}^{(X)}\right]^{-1} \delta \Psi_{\ell, 1}^{(X)}\right] .
\end{aligned}
$$

In order to perform the $\overline{\mathrm{MS}}$ subtraction from the divergent part, we can use

$$
\ln \mathcal{A}^{(X)}=-\frac{1}{2} \operatorname{Tr}\left[\widehat{\mathcal{M}}^{(X)}\right]^{-1} \delta \mathcal{M}+\frac{1}{4} \operatorname{Tr}\left[\widehat{\mathcal{M}}^{(X)}\right]^{-1} \delta \mathcal{M}\left[\widehat{\mathcal{M}}^{(X)}\right]^{-1} \delta \mathcal{M}+\mathcal{O}\left(\delta \mathcal{M}^{3}\right),
$$

to obtain

$$
\begin{aligned}
& s_{1}^{(X)}=\frac{1}{2} \operatorname{Tr}\left[\widehat{\mathcal{M}}^{(X)}\right]^{-1} \delta \mathcal{M}, \\
& s_{2}^{(X)}=-\frac{1}{4} \operatorname{Tr}\left[\widehat{\mathcal{M}}^{(X)}\right]^{-1} \delta \mathcal{M}\left[\widehat{\mathcal{M}}^{(X)}\right]^{-1} \delta \mathcal{M} .
\end{aligned}
$$

These quantities can be calculated by using the ordinary diagrammatic method. We calculate them adopting dimensional regularization, and perform $\overline{\mathrm{MS}}$ subtraction to obtain the renormalized quantities, $s_{1, \overline{\mathrm{MS}}}^{(X)}$ and $s_{2, \overline{\mathrm{MS}}}^{(X)}$; their explicit forms are given in appendix C. ${ }^{11}$

The prefactor after the $\overline{\mathrm{MS}}$ subtraction can be obtained as

$$
\ln \mathcal{A}_{\overline{\mathrm{MS}}}^{(X)}=\left[\ln \mathcal{A}+s_{1}^{(X)}+s_{2}^{(X)}\right]-s_{1, \overline{\mathrm{MS}}}^{(X)}-s_{2, \overline{\mathrm{MS}}}^{(X)},
$$

where $s_{1}^{(X)}$ and $s_{2}^{(X)}$ in the square bracket should be understood as those given in eqs. (7.9) and (7.10), respectively. Notice that the quantity in the square bracket can be evaluated order by order in $\ell$. At a large $\ell$, the contribution behaves as $\ell^{-2}$ and the sum over $\ell$ converges. One may truncate higher $\ell$, or fit the terms with large enough $\ell$ and sum them up for better precision.

\footnotetext{
${ }^{11}$ There was an error in the calculation of the counterterms in [34], which is corrected in the expression in appendix $\mathrm{C}$.
} 


\section{Conclusions and discussion}

In this paper, we have studied the decay rate of the false vacuum in gauge theory for the case with a multi-field bounce. If more than one scalar fields contribute to the bounce, the mixing among the gauge fields and the scalars becomes $r$-dependent, making it complicated to calculate the one-loop contributions to the decay rate. We have extended the results of $[33,34]$ to perform a gauge invariant calculation of the decay rate at the one-loop level. The one-loop contributions to the decay rate, which are denoted as $\mathcal{A}$ in this article, are given by the ratio of the functional determinants of the fluctuation operators around the bounce and those around the false vacuum (see eqs. (2.61) and (2.62)). Using the fact that the functional determinant of the fluctuation operator, $\mathcal{M}^{(X)}$, can be related to the asymptotic behavior of the function, $\psi^{(X)}$, obeying $\mathcal{M}^{(X)} \psi^{(X)}=0$, we have derived an expression of the decay rate at the one-loop level, which is manifestly independent of the gauge parameter, $\xi$. In particular, we have discussed how the functional determinant of $\mathcal{M}^{\left(A_{\mu} \varphi\right)}$, i.e. the fluctuation operator for the gauge and scalar fields, can be evaluated in a gauge invariant way. Our main results are summarized in section 6 .

In our study, we have worked in both the Fermi gauge and the background gauge. We have shown that the Fermi gauge has an advantage to show the gauge invariance of the result especially when there exist gauge zero modes. The path integral over the gauge zero modes should be replaced by the integration over the gauge volume; the rule of the replacement can be straightforwardly obtained in the Fermi gauge. The background gauge has, however, an advantage in numerically calculating the decay rate because of the better convergence. In order to utilize the calculation in the background gauge, we derived a prescription to translate the result in the background gauge to that in the Fermi gauge.

We have also discussed how we can remove ultraviolet divergences from the decay rate. We have shown a procedure to subtract the divergences from the one-loop results and to perform the renormalization in the $\overline{\mathrm{MS}}$ scheme (see section 7 ).

Our results apply to various types of models with many scalar fields having gauge charges; in such models, it is often the case that there shows up a true vacuum whose energy density is lower than that of the electroweak vacuum. Our results can be used to calculate the decay rate of the electroweak vacuum in such models. Phenomenological application of our results may be discussed elsewhere.

\section{Acknowledgments}

The work of SC is supported by JSPS KAKENHI grant. The work of TM is supported by JSPS KAKENHI grant Nos. 16H06490 and 18K03608. The work of YS is supported by JSPS KAKENHI grant No. 16H06492. 


\section{A Evaluation of determinants}

\section{A.1 Alternative fluctuation operators}

One of the important steps in our calculation is to rewrite $\operatorname{det} \Psi_{\ell}^{(S L \varphi)}$ using $\eta$ and $\lambda$ at $r \sim \infty$. Since the fluctuation matrices approach those at the false vacuum as $r \rightarrow \infty$, one may expect that the behavior of $\Psi_{\ell}^{(S L \varphi)}$ at $r \sim \infty$ is the same as that of the solution at the false vacuum, $\widehat{\Psi}_{\ell}^{(S L \varphi)}$. However, it is not always the case because they are related as

$$
\Psi_{\ell}^{(S L \varphi)}(r \rightarrow \infty)=\widehat{\Psi}_{\ell}^{(S L \varphi)}(r) G(r),
$$

where $G$ is a square matrix. Since the elements of $\widehat{\Psi}_{\ell}^{(S L \varphi)}$ generally become hierarchical as $r \rightarrow \infty$, larger elements easily contaminate smaller elements due to the mixing induced by $G$. The crucial problem here is that the contamination cannot be avoided just by taking a linear combination of the solutions since $G$ is $r$-dependent. Such contamination leads to a severe cancellation when we calculate the determinant of $\Psi_{\ell}^{(S L \varphi)}$. In the following, we discuss a way to avoid this problem.

We first define the projection operators as

$$
\begin{aligned}
P_{H} & =V_{H} V_{H}^{T}, \\
P_{G} & =M\left(M^{T} M\right)^{-1} M^{T},
\end{aligned}
$$

which satisfy $P_{H}+P_{G}=I_{\varphi}$ and $P_{H} P_{G}=P_{G} P_{H}=0$. Then, we define $r$-dependent orthogonal matrices $O_{\varphi}(r)$ and $O_{G}(r)$ having the following properties:

$$
\begin{aligned}
& O_{\varphi}^{T}(r) M(r) O_{G}(r)=\left(\begin{array}{c}
W(r) \\
0
\end{array}\right)\left\{\begin{array}{l}
n_{G} \\
n_{H}
\end{array}=(\begin{array}{cc}
W_{B}(r) & 0 \\
0 & W_{U}(r) \\
0 & 0
\end{array} \underbrace{0}_{n_{U}},\} \begin{array}{l}
n_{B} \\
n_{U}, \\
n_{H}
\end{array}\right. \\
& O_{\varphi}^{T}(r) P_{H}(r) \Omega(r) P_{H}(r) O_{\varphi}(r)=(\begin{array}{cc}
0 & 0 \\
0 & n_{n_{G}}^{m^{2}(r)}
\end{array} \underbrace{m_{H}}_{n_{H}}, n_{G},
\end{aligned}
$$

where $m^{2}, W, W_{B}$ and $W_{U}$ are diagonal matrices. Since we work in the field basis given in eqs. (2.28) and (2.29), we can take

$$
\lim _{r \rightarrow \infty} O_{\varphi}(r)=I_{\varphi}, \quad \lim _{r \rightarrow \infty} O_{G}(r)=I_{G} .
$$

The basic idea is to use the following matrices instead of $M$ and $\Omega$.

$$
\begin{aligned}
& M_{\mathrm{alt}}=\left\{\begin{array}{ll}
M, & r \ll r_{*} \\
O_{\varphi}^{T} M O_{G}, & r \gg r_{*}
\end{array},\right. \\
& \Omega_{\mathrm{alt}}= \begin{cases}\Omega, & r \ll r_{*} \\
O_{\varphi}^{T} P_{H} \Omega P_{H} O_{\varphi}+O_{\varphi}^{T} P_{G} \Omega P_{G} O_{\varphi}, & r \gg r_{*}\end{cases}
\end{aligned}
$$


Here, $r_{*}$ is taken to be much larger than the typical scale of the bounce. Then, the behavior of the solution at $r \rightarrow \infty$ can be understood easily since the fluctuation operators become block-diagonalized. The behavior at $r \sim r_{*}$ will be defined in appendix B.

The decomposed solution in section 3 cannot be used as it is since $M_{\text {alt }}$ does not satisfy eqs. (2.15) and (2.16) for $r \gtrsim r_{*}$. However, we find that the solution becomes exact if we add extra scalar mass terms to the fluctuation operators as

$$
\begin{gathered}
\mathcal{M}_{\ell, \text { alt }}^{(S L \varphi)}=\left.\mathcal{M}_{\ell}^{(S L \varphi)}\right|_{M \rightarrow M_{\mathrm{alt}}, \Omega \rightarrow \Omega_{\mathrm{alt}}+\delta \Omega} \\
\mathcal{M}_{0, \text { alt }}^{(S \varphi)}=\left.\mathcal{M}_{0}^{(S \varphi)}\right|_{M \rightarrow M_{\mathrm{alt}}, \Omega \rightarrow \Omega_{\mathrm{alt}}+\delta \Omega}
\end{gathered}
$$

where

$$
\delta \Omega=-M_{\mathrm{alt}}\left(M_{\mathrm{alt}}^{T} M_{\mathrm{alt}}\right)^{-1} \varepsilon^{T},
$$

with

$$
\varepsilon=-\Delta_{0} M_{\text {alt }}+\Omega_{\text {alt }} M_{\text {alt }}
$$

Then, the solutions of

$$
\mathcal{M}_{\ell, \text { alt }}^{(S L \varphi)} \Psi_{\ell, \text { alt }}^{(S L \varphi)}=0
$$

and

$$
\mathcal{M}_{0, \text { alt }}^{(S \varphi)} \Psi_{0, \text { alt }}^{(S \varphi)}=0
$$

are given by eqs. (3.2)-(3.6) and (3.13) with replacing $M \rightarrow M_{\text {alt }}$ and $\Omega \rightarrow \Omega_{\text {alt }}$. Since the fluctuation operators are not modified for $r \lesssim r_{*}$, we take

$$
\begin{aligned}
\Psi_{\ell, \text { alt }}^{(S L \varphi)}\left(r \lesssim r_{*}\right) & =\Psi_{\ell}^{(S L \varphi)}\left(r \lesssim r_{*}\right), \\
\Psi_{0, \text { alt }}^{(S \varphi)}\left(r \lesssim r_{*}\right) & =\Psi_{0}^{(S \varphi)}\left(r \lesssim r_{*}\right) .
\end{aligned}
$$

Similarly, we define the solutions, $\Psi_{\ell, \text { alt }}^{(c \bar{c})}$ and $\Psi_{\ell \text {,alt }}^{(\eta \lambda)}$, which satisfy the same differential equations as $\Psi_{\ell}^{(c \bar{c})}$ and $\Psi_{\ell}^{(\eta \lambda)}$ but with $M \rightarrow M_{\text {alt }}$ and $\Omega \rightarrow \Omega_{\text {alt }}$.

For later convenience, we also define

$$
\begin{aligned}
& P_{G, \text { alt }}=M_{\text {alt }}\left(M_{\text {alt }}^{T} M_{\text {alt }}\right)^{-1} M_{\text {alt }}^{T}, \\
& P_{H, \text { alt }}=I_{\varphi}-P_{G, \text { alt }} .
\end{aligned}
$$

The deformation at $r \sim r_{*}$ should be smooth enough and the decomposition of the solutions should be well-defined for the entire region of $r$. In addition, we need to ensure the use of these alternative fluctuation operators does not affect the final results. We will discuss these issues in appendix B. 


\section{A.2 Evaluation of solutions for $\ell>0$}

\section{A.2.1 Behavior at infinity}

Here, we evaluate $\Psi_{\ell \text {,alt }}^{(S L \varphi)}$ at $r \rightarrow \infty$. For this purpose, we can use the fact that $\Psi_{\ell, \text { alt }}^{(c \bar{c})}$ and $\Psi_{\ell, \text { alt }}^{(\eta \lambda)}$ behave as follows:

$$
\Psi_{\ell, \text { alt }}^{(c \bar{c})}(r \rightarrow \infty) \simeq\left(\begin{array}{cc}
\frac{e^{\sqrt{\xi} W_{B} r}}{r^{3 / 2}} I_{B} & 0 \\
0 & r^{\ell} I_{U}
\end{array}\right) \mathcal{T}_{\ell}^{(c \bar{c})},
$$

and

$$
\Psi_{\ell, \text { alt }}^{(\eta \lambda)}(r \rightarrow \infty) \simeq\left(\begin{array}{ccc}
\frac{e^{W_{B} r}}{r^{3 / 2}} I_{B} & 0 & 0 \\
0 & \frac{1}{r^{2}} I_{U} & 0 \\
0 & 0 & \frac{e^{m r}}{r^{3 / 2}} I_{H}
\end{array}\right) \mathcal{T}_{\ell}^{(\eta \lambda)},
$$

where $\mathcal{T}_{\ell}^{(c \bar{c})}$ and $\mathcal{T}_{\ell}^{(\eta \lambda)}$ are constant square matrices.

We examine the behavior of each type of the solutions at $r \rightarrow \infty$ below.

- Type 1

In the Fermi gauge, $\chi^{(1)}=r^{\ell} I_{G}$. Then, from eq. (3.2),

$$
\Psi_{\ell, \text { alt }}^{(S L \varphi)(1)}(r \rightarrow \infty) \simeq\left(\begin{array}{c}
\frac{\ell}{r} I_{G} \\
\frac{L}{r} I_{G} \\
W \\
0
\end{array}\right) \begin{cases}n_{G} \\
n_{G} & \\
n_{G} & r^{\ell} . \\
n_{G} & \\
n_{H} & \end{cases}
$$

In the background gauge, $\chi^{(1)}=\Psi_{\ell \text { alt }}^{(c \bar{c})}$. Using eq. (3.2),

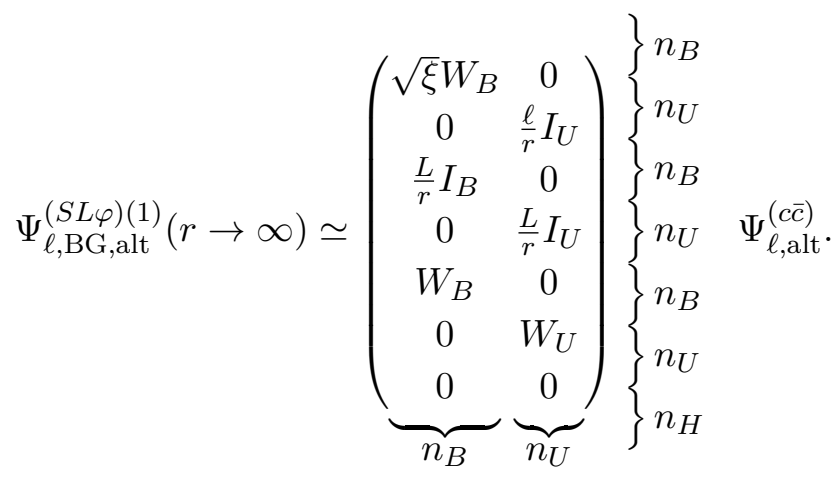

- Type 2

Since $P_{H, \text { alt }} M_{\text {alt }}^{\prime}=0$ for $r \gtrsim r_{*}, \lambda^{(2)}=0$ from eq. (3.6). In the Fermi gauge, $\zeta^{(2)}=r^{\ell}$, and

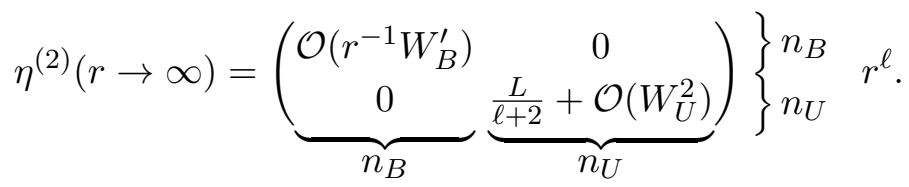


Then, from eqs. (3.8)-(3.10),

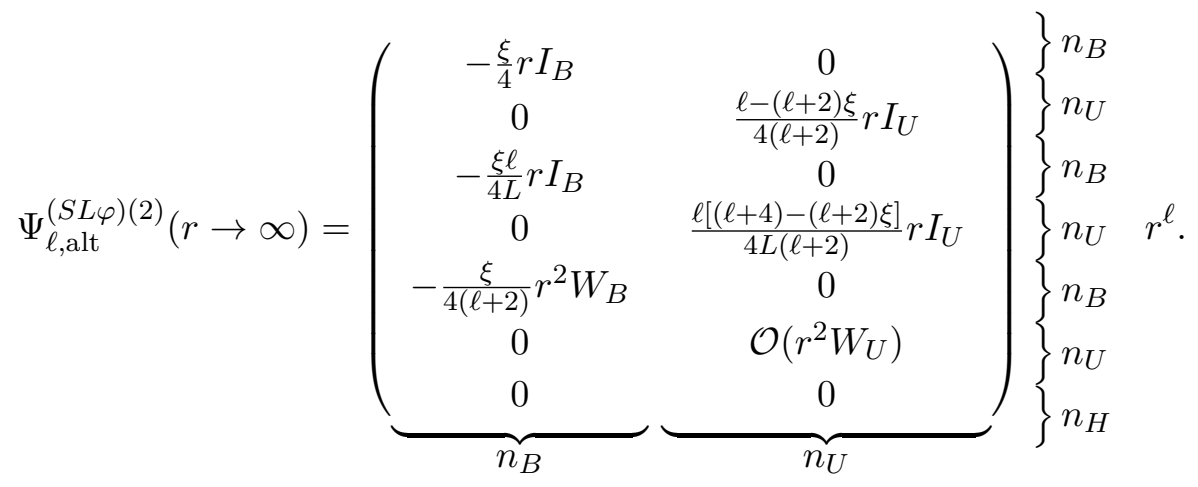

In the background gauge, $\zeta^{(2)}=\Psi_{\ell, \text { alt }}^{(c \bar{c})}$. Then,

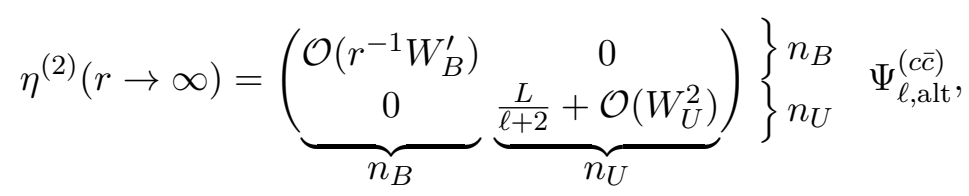

and

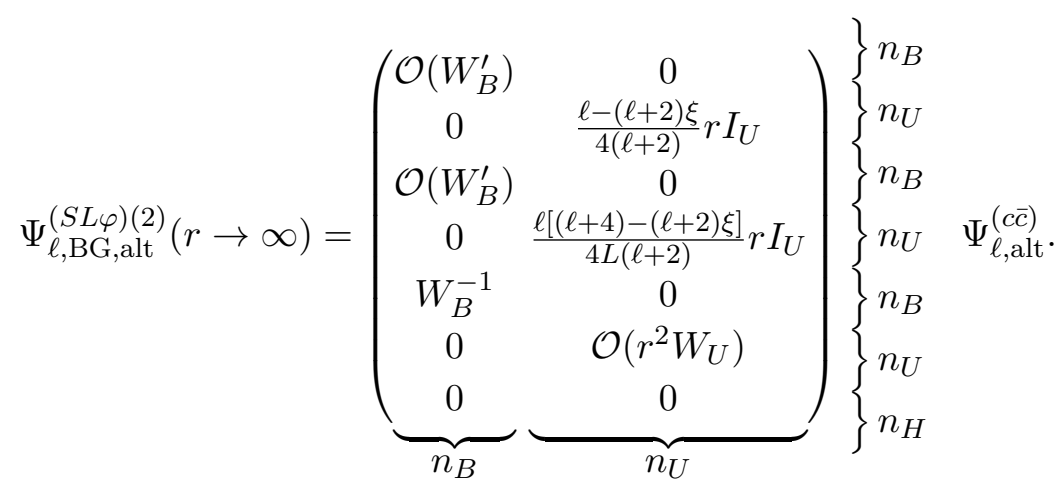

- Type 3

When gauge bosons become massive at the false vacuum, it is convenient to use the following relations:

$$
\begin{aligned}
\Delta_{\ell}\left(r \Psi_{\ell}^{(\mathrm{top})}\right) & =L \eta-\frac{\xi}{r} \partial_{r} r^{2} Y \\
\Psi_{\ell}^{(\mathrm{mid})} & =\frac{1}{L r^{2}} \partial_{r} r^{2}\left(r \Psi_{\ell}^{(\mathrm{top})}\right)+\frac{r \xi}{L} Y, \\
\Psi_{\ell}^{(\mathrm{bot})} & =M \chi+\lambda+M\left(M^{T} M\right)^{-1} \zeta
\end{aligned}
$$

where

$$
Y=\left\{\begin{array}{ll}
\zeta & : \text { Fermi } \\
-M^{T} M \chi & : \text { BG }
\end{array} .\right.
$$


Using eqs. (3.3) and (3.8)-(3.10) as well as the above relations,

$$
\Psi_{\ell, \text { alt }}^{(S L \varphi)(3)}(r \rightarrow \infty)=\left(\begin{array}{ccc}
\frac{L}{r} W_{B}^{-2} & 0 & 0 \\
0 & -\frac{r}{L} I_{U} & 0 \\
W_{B}^{-1} & 0 & 0 \\
0 & -\frac{2 r}{L^{2}} I_{U} & 0 \\
\mathcal{O}\left(r^{-1} W_{B}^{\prime}\right) & 0 & 0 \\
0 & \frac{L}{2 r}\left(W_{U}^{\prime}\right)^{-1} & 0 \\
0 & \underbrace{0}_{n_{B}} & \underbrace{I_{H}}_{n_{H}}
\end{array}\right\}\left\{\begin{array}{l}
n_{B} \\
n_{U} \\
n_{B} \\
n_{U} \quad \Psi_{\ell, \text { alt }}^{(\eta \lambda)} . \\
n_{B} \\
n_{U} \\
n_{H}
\end{array}\right.
$$

\section{A.2.2 Translational zero modes}

Here, we discuss the translational zero modes. The following discussion applies to both the Fermi gauge and the background gauge. With the alternative fluctuation operator, $\mathcal{M}_{1, \text { alt }}^{(S L)}$, the existence of the translational zero modes is not guaranteed; $\operatorname{det} \Psi_{1, \text { alt }}^{(S L)}(r \rightarrow \infty)$ can be non-vanishing. However, it does not cause a problem since it approaches zero as $r_{*} \rightarrow \infty$ and we always take $\nu \rightarrow 0$ after $r_{*} \rightarrow \infty$.

Let us start with the original fluctuation matrix (the one without "alt"). To construct the solution explicitly, we regularize the translational zero modes using

$$
\left.\mathcal{M}_{1, \mathrm{reg}}^{(S L \varphi)}=\mathcal{M}_{1}^{(S L \varphi)}+\nu(\underbrace{0}_{2 n_{G}} \underbrace{P_{H}}_{n_{\varphi}})\right\} n_{\varphi},
$$

instead of $\mathcal{M}_{1}^{(S L \varphi)}+\nu$. Since

$$
\mathcal{M}_{1, \text { reg }}^{(S L \varphi)} \psi^{(\mathrm{tr})}=\nu \psi^{(\mathrm{tr})},
$$

we can use the same discussion for the zero modes as in section 5 . Then, we define its alternative as

$$
\mathcal{M}_{1, \text { reg,alt }}^{(S L \varphi)}=\left.\mathcal{M}_{1, \text { reg }}^{(S L \varphi)}\right|_{M \rightarrow M_{\text {alt }}, \Omega \rightarrow \Omega_{\text {alt }}+\delta \Omega, P_{H} \rightarrow P_{H, \text { alt }}} .
$$

Since $\psi^{(\operatorname{tr})}$ is included in $\Psi_{1}^{(S L \varphi)(3)}$, it is enough to calculate

$$
\mathcal{M}_{1, \text { reg,alt }}^{(S L \varphi)} \check{\Psi}_{1, \text { alt }}^{(S L \varphi)(3)}=-\Psi_{1, \text { alt }}^{(S L \varphi)(3)} .
$$

The solution is given by

$$
\check{\Psi}_{1, \text { alt }}^{(S L \varphi)(3)}=\left.\Psi_{1, \text { alt }}^{(S L \varphi)(3)}\right|_{\Psi_{1, \text { alt }}^{(\eta \lambda)} \rightarrow \check{\Psi}_{1, \text { alt }}^{(\eta \lambda)}},
$$

where

$$
\check{\Psi}_{1, \text { alt }}^{(\eta \lambda)}=\left(\begin{array}{c}
\check{\eta} \\
V_{H, \text { alt }}^{T} \check{\lambda}
\end{array}\right) .
$$


Here, $\check{\eta}$ and $\check{\lambda}$ satisfy

$$
\begin{aligned}
& \Delta_{1} \check{\eta}=\left.\Delta_{1} \eta\right|_{\eta \rightarrow \check{\eta}, \zeta \rightarrow 0, \lambda \rightarrow \check{\lambda}, M \rightarrow M_{\mathrm{alt}}, \Omega \rightarrow \Omega_{\mathrm{alt}}}, \\
& \Delta_{1} \check{\lambda}=\left.\Delta_{1} \lambda\right|_{\eta \rightarrow \check{\eta}, \zeta \rightarrow 0, \lambda \rightarrow \check{\lambda}, M \rightarrow M_{\mathrm{alt}}, \Omega \rightarrow \Omega_{\mathrm{alt}}}+\left(\begin{array}{lll}
0 & 0 & V_{H, \mathrm{alt}}
\end{array}\right) \Psi_{1, \mathrm{alt}}^{(S L \varphi)} .
\end{aligned}
$$

\section{A.2.3 Functional determinant $(\ell>1)$}

Here, we calculate the determinant of the solutions obtained above.

In the Fermi gauge,

$$
\begin{aligned}
\operatorname{det} \Psi_{\ell, \text { alt }}^{(S L \varphi)}(r \rightarrow \infty) & =\operatorname{det}\left(\Psi_{\ell, \text { alt }}^{(S L \varphi)(1)} \Psi_{\ell, \text { alt }}^{(S L \varphi)(2)} \Psi_{\ell, \text { alt }}^{(S L \varphi)(3)}\right) \\
& \simeq\left(-\frac{\xi r}{2(\ell+2)}\right)^{n_{B}}\left(\frac{\ell[\ell+\xi(\ell+2)]}{4(\ell+2) r}\right)^{n_{U}} r^{2 \ell n_{G}} \frac{\operatorname{det} \Psi_{\ell, \text { alt }}^{(\eta \lambda)}}{\operatorname{det} W_{U}^{\prime}}
\end{aligned}
$$

and

$$
\begin{aligned}
\operatorname{det} \widehat{\Psi}_{\ell}^{(S L \varphi)}(r \rightarrow \infty) & =\operatorname{det} \widehat{\Psi}_{\ell}^{(B)} \operatorname{det} \widehat{\Psi}_{\ell}^{(U)} \operatorname{det} \widehat{\Psi}_{\ell}^{(\lambda)} \\
& \simeq\left(-\frac{\xi r}{2(\ell+2)}\right)^{n_{B}} r^{2 \ell n_{B}} \operatorname{det} \widehat{\Psi}_{\ell}^{(\eta)}\left(-\frac{\ell+\xi(\ell+2)}{2(\ell+2)} r^{2 \ell}\right)^{n_{U}} \operatorname{det} \widehat{\Psi}_{\ell}^{(\lambda)}
\end{aligned}
$$

Thus,

$$
\frac{\operatorname{det} \Psi_{\ell, \mathrm{alt}}^{(S L \varphi)}(r \rightarrow \infty)}{\operatorname{det} \widehat{\Psi}_{\ell}^{(S L \varphi)}(r \rightarrow \infty)}=\left(-\frac{\ell}{2 r_{\infty}}\right)^{n_{U}} \frac{\operatorname{det} \Psi_{\ell, \mathrm{alt}}^{(\eta \lambda)}}{\operatorname{det} \widehat{\Psi}_{\ell}^{(\eta)} \operatorname{det} \widehat{\Psi}_{\ell}^{(\lambda)} \operatorname{det} W_{U}^{\prime}} .
$$

Keeping only the leading terms in $r$, we can interchange $r \rightarrow \infty$ and $r_{*} \rightarrow \infty$ and

$$
\frac{\operatorname{det} \Psi_{\ell}^{(S L \varphi)}(r \rightarrow \infty)}{\operatorname{det} \widehat{\Psi}_{\ell}^{(S L \varphi)}(r \rightarrow \infty)}=\left(-\frac{\ell}{2 r_{\infty}}\right)^{n_{U}} \frac{\operatorname{det} \Psi_{\ell}^{(\eta \lambda)}}{\operatorname{det} \widehat{\Psi}_{\ell}^{(\eta)} \operatorname{det} \widehat{\Psi}_{\ell}^{(\lambda)} \operatorname{det} W_{U}^{\prime}} .
$$

In the background gauge,

$$
\begin{aligned}
\operatorname{det} \Psi_{\ell, \mathrm{BG}, \mathrm{alt}}^{(S L \varphi)}(r \rightarrow \infty) & =\operatorname{det}\left(\Psi_{\ell, \mathrm{BG}, \mathrm{alt}}^{(S L \varphi)(1)} \Psi_{\ell, \mathrm{BG}, \mathrm{alt}}^{(S L \varphi)(2)} \Psi_{\ell, \mathrm{BG}, \mathrm{alt}}^{(S L \varphi)(3)}\right) \\
& \simeq(-\sqrt{\xi})^{n_{B}} \frac{1}{\operatorname{det} \widehat{W}}\left(\frac{\ell[\ell+\xi(\ell+2)]}{4(\ell+2) r}\right)^{n_{U}} \frac{\left[\operatorname{det} \Psi_{\ell, \text { alt }}^{(c \bar{c})}\right]^{2} \operatorname{det} \Psi_{\ell, \mathrm{alt}}^{(\eta \lambda)}}{\operatorname{det} W_{U}^{\prime}}
\end{aligned}
$$

and

$$
\begin{aligned}
\operatorname{det} & \widehat{\Psi}_{\ell, \mathrm{BG}}^{(S L \varphi)}(r \rightarrow \infty)=\operatorname{det} \widehat{\Psi}_{\ell}^{(B)} \operatorname{det} \widehat{\Psi}_{\ell}^{(U)} \operatorname{det} \widehat{\Psi}_{\ell}^{(\lambda)} \\
& \simeq(-\sqrt{\xi})^{n_{B}} \frac{1}{\operatorname{det} \widehat{W}}\left[r^{-\ell n_{U}} \operatorname{det} \widehat{\Psi}_{\ell}^{(c \bar{c})}\right]^{2} \operatorname{det} \widehat{\Psi}_{\ell}^{(\eta)}\left(-\frac{\ell+\xi(\ell+2)}{2(\ell+2)} r^{2 \ell}\right)^{n_{U}} \operatorname{det} \widehat{\Psi}_{\ell}^{(\lambda)} .
\end{aligned}
$$

Here, we have used $W_{B}(r \rightarrow \infty)=\widehat{W}$. Thus,

$$
\frac{\operatorname{det} \Psi_{\ell, \mathrm{BG}, \text { alt }}^{(S L \varphi)}(r \rightarrow \infty)}{\operatorname{det} \widehat{\Psi}_{\ell, \mathrm{BG}}^{(S L \varphi)}(r \rightarrow \infty)}=\lim _{r \rightarrow \infty}\left(-\frac{\ell}{2 r_{\infty}}\right)^{n_{U}} \frac{\operatorname{det} \Psi_{\ell, \text { alt }}^{(\eta \lambda)}}{\operatorname{det} \widehat{\Psi}_{\ell}^{(\eta)} \operatorname{det} \widehat{\Psi}_{\ell}^{(\lambda)} \operatorname{det} W_{U}^{\prime}}\left(\frac{\operatorname{det} \Psi_{\ell \text { alt }}^{(c \bar{c})}}{\operatorname{det} \widehat{\Psi}_{\ell}^{(c \bar{c})}}\right)^{2},
$$


and hence

$$
\frac{\operatorname{det} \Psi_{\ell, \mathrm{BG}}^{(S L \varphi)}(r \rightarrow \infty)}{\operatorname{det} \widehat{\Psi}_{\ell, \mathrm{BG}}^{(S L \varphi)}(r \rightarrow \infty)}=\left(-\frac{\ell}{2 r_{\infty}}\right)^{n_{U}} \frac{\operatorname{det} \Psi_{\ell}^{(\eta \lambda)}}{\operatorname{det} \widehat{\Psi}_{\ell}^{(\eta)} \operatorname{det} \widehat{\Psi}_{\ell}^{(\lambda)} \operatorname{det} W_{U}^{\prime}}\left(\frac{\operatorname{det} \Psi_{\ell}^{(c \bar{c})}}{\operatorname{det} \widehat{\Psi}_{\ell}^{(c \bar{c})}}\right)^{2} .
$$

From eqs. (4.5), (4.7) and (4.10),

$$
\lim _{r \rightarrow 0} \frac{\operatorname{det} \Psi_{\ell}^{(S L \varphi)}(r)}{\operatorname{det} \widehat{\Psi}_{\ell}^{(S L \varphi)}(r)}=\frac{\operatorname{det} \widehat{W}}{\sqrt{\operatorname{det} M_{0}^{T} M_{0}}},
$$

independently of the choice of the gauge fixing. Taking the ratio between the determinants with $r \rightarrow 0$ and $r \rightarrow \infty$, we get eq. (4.32).

\section{A.2.4 Functional determinant $(\ell=1)$}

In the Fermi gauge,

$$
\begin{aligned}
\operatorname{det} \Psi_{1, \text { reg,alt }}^{(S L \varphi)}(\nu ; r \rightarrow \infty)= & \operatorname{det}\left(\Psi_{1, \text { alt }}^{(S L \varphi)(1)} \Psi_{1, \text { alt }}^{(S L \varphi)(2)} \Psi_{1, \text { alt }}^{(S L \varphi)(3)}+\nu \check{\Psi}_{1, \text { alt }}^{(S L \varphi)(3)}\right) \\
\simeq & \left(-\frac{\xi r}{6}\right)^{n_{B}}\left(\frac{1+3 \xi}{12 r}\right)^{n_{U}} r^{2 n_{G}} \frac{\operatorname{det}\left[\Psi_{1, \text { alt }}^{(\eta \lambda)}+\nu \check{\Psi}_{1, \text { alt }}^{(\eta \lambda)}\right]}{\operatorname{det} W_{U}^{\prime}} \\
& +\mathcal{O}\left(\nu^{2}\right),
\end{aligned}
$$

and

$$
\operatorname{det} \widehat{\Psi}_{1, \text { reg }}^{(S L \varphi)}(\nu ; r \rightarrow \infty) \simeq\left(-\frac{\xi r}{6}\right)^{n_{B}} r^{2 n_{B}} \operatorname{det} \widehat{\Psi}_{1}^{(\eta)}\left(-\frac{1+3 \xi}{6} r^{2}\right)^{n_{U}} \operatorname{det} \widehat{\Psi}_{1}^{(\lambda)}+\mathcal{O}(\nu) .
$$

Thus,

$$
\frac{\operatorname{det} \Psi_{1, \text { reg,alt }}^{(S L \varphi)}(\nu ; r \rightarrow \infty)}{\operatorname{det} \widehat{\Psi}_{1, \text { reg }}^{(S L \varphi)}(\nu ; r \rightarrow \infty)}=\left(-\frac{1}{2 r_{\infty}}\right)^{n_{U}} \frac{\operatorname{det}\left[\Psi_{1, \text { alt }}^{(\eta \lambda)}+\nu \check{\Psi}_{1, \text { alt }}^{(\eta \lambda)}\right]}{\operatorname{det} \widehat{\Psi}_{1}^{(\eta)} \operatorname{det} \widehat{\Psi}_{1}^{(\lambda)} \operatorname{det} W_{U}^{\prime}}+\mathcal{O}\left(\nu^{2}, \mathcal{E} \nu\right),
$$

where

$$
\mathcal{E}=\lim _{\nu \rightarrow 0} \frac{\operatorname{det} \Psi_{1, \text { reg,alt }}^{(S L \varphi)}(\nu ; r \rightarrow \infty)}{\operatorname{det} \widehat{\Psi}_{1, \text { reg }}^{(S L \varphi)}(\nu ; r \rightarrow \infty)} .
$$

Notice that $\mathcal{E}$ can be non-vanishing when the translational zero modes disappear in the alternative fluctuation operator. As $r_{*} \rightarrow \infty, \mathcal{E}$ should approach zero. Keeping only the leading terms in $r$, we can interchange $r \rightarrow \infty$ and $r_{*} \rightarrow \infty$. Then,

$$
\lim _{\nu \rightarrow 0} \frac{1}{\nu} \frac{\operatorname{det} \Psi_{1}^{(S L \varphi)}(\nu ; r \rightarrow \infty)}{\operatorname{det} \widehat{\Psi}_{1}^{(S L \varphi)}(\nu ; r \rightarrow \infty)}=\lim _{\nu \rightarrow 0} \frac{1}{\nu}\left(-\frac{1}{2 r_{\infty}}\right)^{n_{U}} \frac{\operatorname{det}\left[\Psi_{1}^{(\eta \lambda)}+\nu \check{\Psi}_{1}^{(\eta \lambda)}\right]}{\operatorname{det} \widehat{\Psi}_{1}^{(\eta)} \operatorname{det} \widehat{\Psi}_{1}^{(\lambda)} \operatorname{det} W_{U}^{\prime}} .
$$

In the background gauge,

$$
\begin{aligned}
& \operatorname{det} \Psi_{1, \text { reg,BG,alt }}^{(S L \varphi)}(\nu ; r \rightarrow \infty)=\operatorname{det}\left(\Psi_{1, \mathrm{BG}, \text { alt }}^{(S L \varphi)(1)} \Psi_{1, \mathrm{BG}, \mathrm{alt}}^{(S L \varphi)(2)} \Psi_{1, \mathrm{BG}, \mathrm{alt}}^{(S L \varphi)(3)}+\nu \check{\Psi}_{1, \mathrm{BG}, \text { alt }}^{(S L \varphi)(3)}\right) \\
& \simeq(-\sqrt{\xi})^{n_{B}} \frac{1}{\operatorname{det} \widehat{W}}\left(\frac{1+3 \xi}{12 r}\right)^{n_{U}} \frac{\left[\operatorname{det} \Psi_{1, \mathrm{alt}}^{(c \bar{c})}\right]^{2} \operatorname{det}\left[\Psi_{1, \text { alt }}^{(\eta \lambda)}+\nu \check{\Psi}_{1, \mathrm{alt}}^{(\eta \lambda)}\right]}{\operatorname{det} W_{U}^{\prime}}+\mathcal{O}\left(\nu^{2}\right),
\end{aligned}
$$


and

$$
\begin{aligned}
\operatorname{det} & \widehat{\Psi}_{1, \mathrm{reg}, \mathrm{BG}}^{(S L \varphi)}(\nu ; r \rightarrow \infty)=\operatorname{det} \widehat{\Psi}_{1}^{(B)} \operatorname{det} \widehat{\Psi}_{1}^{(U)} \operatorname{det} \widehat{\Psi}_{1}^{(\lambda)} \\
& \simeq(-\sqrt{\xi})^{n_{B}} \frac{1}{\operatorname{det} \widehat{W}}\left[r^{-1 n_{U}} \operatorname{det} \widehat{\Psi}_{1}^{(c \bar{c})}\right]^{2} \operatorname{det} \widehat{\Psi}_{1}^{(\eta)}\left(-\frac{1+3 \xi}{6} r^{2}\right)^{n_{U}} \operatorname{det} \widehat{\Psi}_{1}^{(\lambda)}+\mathcal{O}(\nu) .
\end{aligned}
$$

Here, we have used $W_{B}(r \rightarrow \infty)=\widehat{W}$. Thus,

$$
\begin{aligned}
& \lim _{\nu \rightarrow 0} \frac{1}{\nu} \frac{\operatorname{det} \Psi_{1, \mathrm{reg}, \mathrm{BG}, \text { alt }}^{(S L \varphi)}(\nu ; r \rightarrow \infty)}{\operatorname{det} \widehat{\Psi}_{1, \mathrm{reg}, \mathrm{BG}}^{(S L \varphi)}(\nu ; r \rightarrow \infty)} \\
& \quad=\lim _{\nu \rightarrow 0} \lim _{r \rightarrow \infty} \frac{1}{\nu}\left(-\frac{1}{2 r_{\infty}}\right)^{n_{U}} \frac{\operatorname{det}\left[\Psi_{1, \text { alt }}^{(\eta \lambda)}+\nu \check{\Psi}_{1, \text { alt }}^{(\eta \lambda)}\right]}{\operatorname{det} \widehat{\Psi}_{1}^{(\eta)} \operatorname{det} \widehat{\Psi}_{1}^{(\lambda)} \operatorname{det} W_{U}^{\prime}}\left(\frac{\operatorname{det} \Psi_{1, \text { alt }}^{(c \bar{c})}}{\operatorname{det} \widehat{\Psi}_{1}^{(c \bar{c})}}\right)^{2},
\end{aligned}
$$

and hence

$\lim _{\nu \rightarrow 0} \frac{1}{\nu} \frac{\operatorname{det} \Psi_{1, \mathrm{reg}, \mathrm{BG}}^{(S L \varphi)}(\nu ; r \rightarrow \infty)}{\operatorname{det} \widehat{\Psi}_{1, \mathrm{reg}, \mathrm{BG}}^{(S L \varphi)}(\nu ; r \rightarrow \infty)}=\lim _{\nu \rightarrow 0} \frac{1}{\nu}\left(-\frac{1}{2 r_{\infty}}\right)^{n_{U}} \frac{\operatorname{det}\left[\Psi_{1}^{(\eta \lambda)}+\nu \check{\Psi}_{1}^{(\eta \lambda)}\right]}{\operatorname{det} \widehat{\Psi}_{1}^{(\eta)} \operatorname{det} \widehat{\Psi}_{1}^{(\lambda)} \operatorname{det} W_{U}^{\prime}}\left(\frac{\operatorname{det} \Psi_{1}^{(c \bar{c})}}{\operatorname{det} \widehat{\Psi}_{1}^{(c \bar{c})}}\right)^{2}$.

Taking the ratio between the determinants with $r \rightarrow 0$ and $r \rightarrow \infty$, we obtain eq. (5.68).

\section{A.3 Evaluation of solutions for $\ell=0$}

\section{A.3.1 Behavior at infinity}

The behavior of $\Psi_{0, \text { alt }}^{(c \bar{c})}$ and $\Psi_{0 \text {,alt }}^{(\lambda)}$ is given by

$$
\Psi_{0, \text { alt }}^{(c \bar{c})}(r \rightarrow \infty) \simeq\left(\begin{array}{cc}
\frac{e^{\sqrt{\xi} W_{B^{r}}}}{r^{3 / 2}} I_{B} & 0 \\
0 & I_{U}+\mathcal{O}\left(r^{-2}\right)
\end{array}\right) \mathcal{T}_{0}^{(c \bar{c})},
$$

and

$$
\Psi_{0, \text { alt }}^{(\lambda)}(r \rightarrow \infty) \simeq \frac{e^{m r}}{r^{3 / 2}} I_{H} \mathcal{T}_{0}^{(\lambda)}
$$

where $\mathcal{T}_{0}^{(c \bar{c})}$ and $\mathcal{T}_{0}^{(\lambda)}$ are constant square matrices.

Next, we consider the behavior of $\Psi_{0, \text { alt }}^{(S L)}$ at $r \rightarrow \infty$.

- Type 1

In the Fermi gauge, $\chi^{(1)}=I_{G}$. Then, from eq. (3.13),

$$
\Psi_{0, \text { alt }}^{(S \varphi)(1)}(r \rightarrow \infty) \simeq(\begin{array}{cc}
0 & 0 \\
0 & 0 \\
W_{B} & 0 \\
0 & W_{U} \\
0 & 0
\end{array} \underbrace{0}_{n_{B}}\}\left\{\begin{array}{l}
n_{U} \\
n_{U} \\
n_{B} . \\
n_{U} \\
n_{H}
\end{array}\right.
$$


In the background gauge, $\chi^{(1)}=\Psi_{0, \text { alt }}^{(c \bar{c})}$, and

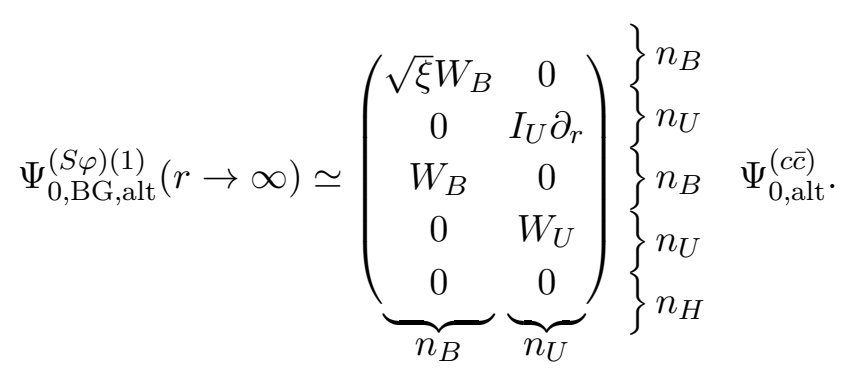

- Type 2

There are some useful relations:

$$
\begin{aligned}
\frac{1}{r^{3}} \partial_{r} r^{3} \Psi_{0}^{(\mathrm{top})} & =-\xi Y, \\
\Psi_{0}^{(\mathrm{bot})} & =\lambda+M \Gamma,
\end{aligned}
$$

where $Y$ is given in eq. (A.30) and $\Gamma$ is a function obeying

$$
\partial_{r} \Gamma=\Psi_{0}^{(\mathrm{top})}+\left(M^{T} M\right)^{-1}\left[\partial_{r} \zeta+2\left(M^{\prime}\right)^{T} \lambda\right] .
$$

In the Fermi gauge, $\zeta^{(2)}=I_{G}$. From eqs. (A.62) and (A.63), we get

$$
\Psi_{0, \text { alt }}^{(S \varphi)(2)}(r \rightarrow \infty) \simeq\left(\begin{array}{c}
-\frac{\xi}{4} r I_{G} \\
-\frac{\xi}{8} r^{2} W \\
0
\end{array}\right)\left\{\begin{array}{l}
n_{G} \\
n_{G}
\end{array}\right\} n_{H} .
$$

In the background gauge, $\zeta^{(2)}=\Psi_{0, \text { alt }}^{(c \bar{c})}$, and

$$
\chi^{(2)}(r \rightarrow \infty) \simeq(\underbrace{\left.\begin{array}{cc}
\mathcal{O}\left(W_{B}^{\prime}\right) & 0 \\
0 & -W_{U}^{-2}
\end{array}\right)}_{n_{B}} \underbrace{-W_{B}}_{n_{U}} \Psi_{U}^{(c \bar{c})} .
$$

The behavior of $W_{U}$ is given by

$$
W_{U}(r \rightarrow \infty) \propto \frac{e^{W_{U}^{\prime}\left(W_{U}\right)^{-1} r}}{r^{3 / 2}} .
$$


From eqs. (A.62) and (A.63), we get

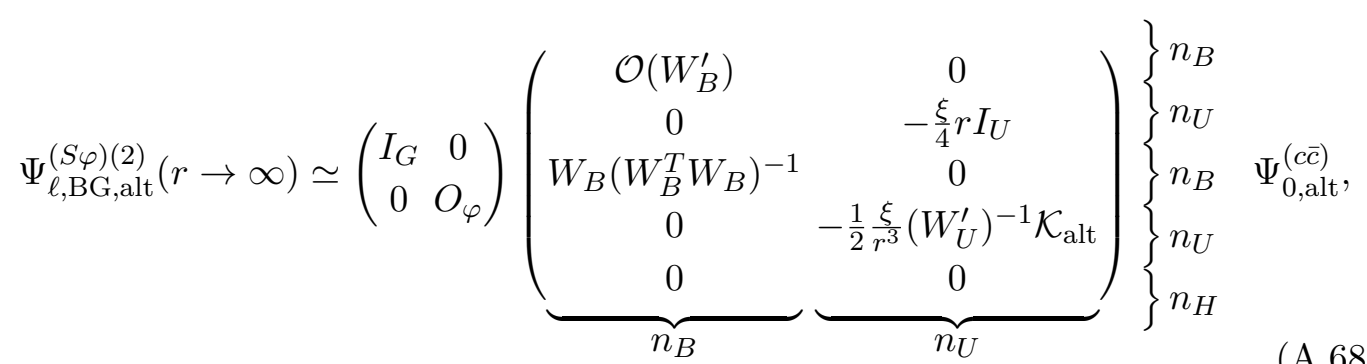

in the background gauge. Here, $\mathcal{K}_{\text {alt }}$ is the $n_{U} \times n_{U}$ matrix defined by

$$
\mathcal{K}_{\text {alt }}=\lim _{r \rightarrow \infty} \frac{r^{3}}{\xi}\left(\begin{array}{ll}
0 & I_{U}
\end{array}\right)\left(\partial_{r} \Psi_{0, \text { alt }}^{(c \bar{c})}\right)\left(\Psi_{0, \text { alt }}^{(c \bar{c})}\right)^{-1}\left(\begin{array}{c}
0 \\
I_{U}
\end{array}\right) .
$$

Notice that its elements are finite and its determinant is non-vanishing.

- Type 3

We can take $\zeta^{(3)}=0$ and $\lambda^{(3)}=\Psi_{0, \text { alt }}^{(\lambda)}$. From eq. (3.3), $\chi^{(3)}(r)=0$ for $r \gtrsim r_{*}$. Then, from eq. (3.13),

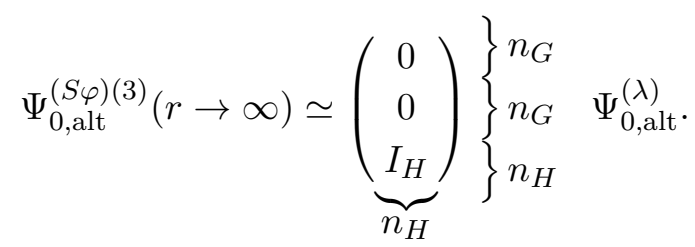

\section{A.3.2 Gauge zero modes}

Here, we discuss the gauge zero modes. Even with the alternative fluctuation operator, there appear gauge zero modes, which are given by

$$
\psi_{\text {alt }}^{(A)}=\left(\begin{array}{c}
0 \\
M_{\text {alt }}
\end{array}\right) \mathcal{U}^{(A)} .
$$

To subtract these zero modes, we need to solve

$$
\mathcal{M}_{0, \text { alt }}^{(S \varphi)} \check{\psi}_{\text {alt }}^{(A)}=-\psi_{\text {alt }}^{(A)} .
$$

The solutions can be obtained by using eqs. (5.33)-(5.36) with the replacement of $M \rightarrow M_{\mathrm{alt}}$ and $\Omega \rightarrow \Omega_{\text {alt }}$.

We define

$$
\left(\begin{array}{c}
Z_{B}^{(A)} \\
Z_{U}^{(A)}
\end{array}\right)\left\{\begin{array}{l}
n_{B} \\
n_{U}
\end{array}=\int d r r^{3} M_{\text {alt }}^{T} M_{\text {alt }} \mathcal{U}^{(A)},\right.
$$


where $Z_{B}^{(A)}$ and $Z_{U}^{(A)}$ become constant at $r \rightarrow \infty$. Then, from eqs. (A.62) and (A.63), we obtain

$$
\check{\Psi}_{0, \text { alt }}^{(S \varphi)(1 U)}(r \rightarrow \infty) \simeq(\underbrace{\frac{\xi}{4 r} Z_{B}^{(A)}}_{n_{U}} \begin{array}{c}
\frac{\xi}{4 r} Z_{U}^{(A)} \\
\frac{\xi}{4} W_{B} Z_{B}^{(A)} \ln r \\
-\frac{1}{2 r^{3}}\left(W_{U}^{\prime}\right)^{-1} Z_{U}^{(A)} \\
0
\end{array})\left\{\begin{array}{l}
n_{B} \\
n_{U} \\
n_{B}, \\
n_{U} \\
n_{H}
\end{array}\right.
$$

with which we define

$$
\check{\Psi}_{0, \text { alt }}^{(S \varphi)(1 U)}=\left(\check{\psi}_{\text {alt }}^{(1)} \cdots \check{\psi}_{\text {alt }}^{\left(n_{U}\right)}\right) .
$$

Let us move on to the background gauge. The gauge zero modes are given by

$$
\psi_{\mathrm{BG}, \mathrm{alt}}^{(A)}=\left(\begin{array}{c}
I_{G} \partial_{r} \\
M_{\mathrm{alt}}
\end{array}\right) \Psi_{0, \mathrm{alt}}^{(c \bar{c})} \mathcal{U}_{\mathrm{BG}}^{(A)},
$$

where

$$
\mathcal{U}_{\mathrm{BG}}^{(A)}=\left[\mathcal{T}_{0}^{(c \bar{c})}\right]^{-1} \mathcal{U}^{(A)}
$$

We need to solve

$$
\mathcal{M}_{0, \mathrm{BG}, \mathrm{alt}}^{(S \varphi)} \check{\psi}_{0, \mathrm{BG}, \mathrm{alt}}^{(A)}=-\psi_{0, \mathrm{BG}, \mathrm{alt}}^{(A)} .
$$

Notably, $\check{\psi}_{0, \mathrm{BG} \text {,alt }}^{(A)}$ can be expressed as eq. (5.33) with $\breve{\zeta}=\check{\lambda}=0$, while $\check{\chi}^{(A)}$ satisfies

$$
\Delta_{0} \check{\chi}^{(A)}=\left.\Delta_{0} \chi\right|_{\chi \rightarrow \check{\chi}^{(A)}, \zeta \rightarrow 0, \lambda \rightarrow 0, M \rightarrow M_{\text {alt }}, \Omega \rightarrow \Omega_{\text {alt }}}+\Psi_{0, \text { alt }}^{(c \bar{C})} \mathcal{U}_{\mathrm{BG}}^{(A)} .
$$

The following relation also holds.

$$
\frac{1}{r^{3}} \partial_{r} r^{3} \check{\Psi}_{0}^{(\mathrm{top})(\mathrm{A})}=\xi M^{T} M \check{\chi}^{(A)}+\Psi_{0}^{(c \bar{c})} \mathcal{U}_{\mathrm{BG}}^{(A)} .
$$

From eq. (A.79),

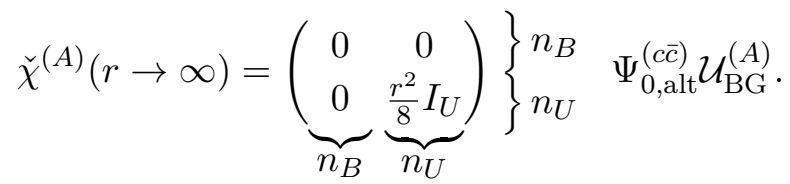

From eqs. (A.80) and (A.63), we obtain

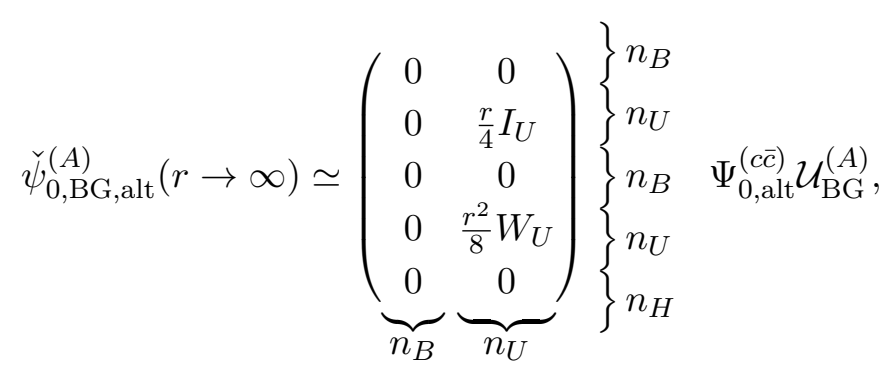


with which we define

$$
\check{\Psi}_{0, \mathrm{BG}, \mathrm{alt}}^{(S \varphi)(1 U)}=\sum_{B} \check{\psi}_{0, \mathrm{BG}, \mathrm{alt}}^{(B)} \mathcal{U}^{(B) T} \mathcal{T}_{0}^{(c \bar{c})}
$$

\section{A.3.3 Functional determinant}

Here, we calculate the determinant of the solutions obtained above.

In the Fermi gauge,

$$
\begin{aligned}
\operatorname{det} \Psi_{0, \text { reg,alt }}^{(S \varphi)}(\nu ; r \rightarrow \infty)= & \operatorname{det}\left(\Psi_{0, \text { alt }}^{(S \varphi)(1 B)} \Psi_{0, \text { alt }}^{(S \varphi)(1 U)}+\nu \check{\Psi}_{0, \text { alt }}^{(S \varphi)(1 U)} \Psi_{0, \text { alt }}^{(S \varphi)(2)} \Psi_{0, \text { alt }}^{(S \varphi)(3)}\right) \\
& +\mathcal{O}\left(\nu^{n_{U}+1}\right) \\
\simeq & \nu^{n_{U}}\left(\frac{\xi}{4} r\right)^{n_{B}} \operatorname{det} W_{B}\left(-\frac{\xi}{8 r^{2}}\right)^{n_{U}} \frac{\operatorname{det} Z_{U}}{\operatorname{det} W_{U}^{\prime}} \operatorname{det} \Psi_{0, \text { alt }}^{(\lambda)}+\mathcal{O}\left(\nu^{n_{U}+1}\right),
\end{aligned}
$$

and

$$
\begin{aligned}
\operatorname{det} \widehat{\Psi}_{0, \text { reg }}^{(S \varphi)}(\nu ; r \rightarrow \infty) & =\operatorname{det} \Psi_{0}^{(B)} \operatorname{det} \Psi_{0}^{(U)} \operatorname{det} \Psi_{0}^{(\lambda)} \\
& \simeq\left(\frac{\xi}{4} r\right)^{n_{B}} \operatorname{det} \widehat{W}\left(\frac{\xi}{4} r\right)^{n_{U}} \operatorname{det} \widehat{\Psi}_{0}^{(\lambda)}+\mathcal{O}(\nu) .
\end{aligned}
$$

Thus,

$$
\frac{1}{\nu^{n_{U}}} \frac{\operatorname{det} \Psi_{0, \text { reg,alt }}^{(S \varphi)}(\nu ; r \rightarrow \infty)}{\operatorname{det} \widehat{\Psi}_{0, \text { reg }}^{(S \varphi)}(\nu ; r \rightarrow \infty)}=\left(-\frac{1}{2 r_{\infty}^{3}}\right)^{n_{U}} \frac{\operatorname{det} Z_{U}}{\operatorname{det} W_{U}^{\prime}} \frac{\operatorname{det} \Psi_{0, \text { alt }}^{(\lambda)}}{\operatorname{det} \widehat{\Psi}_{0}^{(\lambda)}}+\mathcal{O}(\nu),
$$

and

$$
\lim _{\nu \rightarrow 0} \frac{1}{\nu^{n_{U}}} \frac{\operatorname{det} \Psi_{0, \text { reg }}^{(S \varphi)}(\nu ; r \rightarrow \infty)}{\operatorname{det} \widehat{\Psi}_{0, \text { reg }}^{(S \varphi)}(\nu ; r \rightarrow \infty)}=\left(-\frac{1}{2 r_{\infty}^{3}}\right)^{n_{U}} \frac{\operatorname{det} \mathcal{X}_{U}}{\operatorname{det} W_{U}^{\prime}} \frac{\operatorname{det} \Psi_{0}^{(\lambda)}}{\operatorname{det} \widehat{\Psi}_{0}^{(\lambda)}} .
$$

In the background gauge,

$$
\begin{gathered}
\operatorname{det} \Psi_{0, \text { reg,BG,alt }}^{(S L \varphi)}(\nu ; r \rightarrow \infty)=\operatorname{det}\left(\Psi_{0, \mathrm{BG}, \text { alt }}^{(S \varphi)(1)}+\nu \check{\Psi}_{0, \mathrm{BG}, \text { alt }}^{(S \varphi)(1)} \Psi_{0, \mathrm{BG}, \text { alt }}^{(S \varphi)(2)} \Psi_{0, \mathrm{BG}, \text { alt }}^{(S \varphi)(3)}\right)+\mathcal{O}\left(\nu^{n_{U}+1}\right) \\
\simeq \nu^{n_{U}}(\sqrt{\xi})^{n_{B}}\left(-\frac{\xi}{8 r^{2}}\right)^{n_{U}} \frac{\operatorname{det} \mathcal{K}_{\text {alt }}}{\operatorname{det} W_{U}^{\prime}}\left[\operatorname{det} \Psi_{0, \text { alt }}^{(c \bar{c})}\right]^{2} \operatorname{det} \Psi_{\text {alt }}^{(\lambda)}+\mathcal{O}\left(\nu^{n_{U}+1}\right),
\end{gathered}
$$

and

$$
\begin{aligned}
\operatorname{det} \widehat{\Psi}_{0, \mathrm{reg}, \mathrm{BG}}^{(S L \varphi)}(\nu ; r \rightarrow \infty) & =\operatorname{det} \Psi_{0}^{(B)} \operatorname{det} \Psi_{0}^{(U)} \operatorname{det} \Psi_{0}^{(\lambda)} \\
& \simeq(\sqrt{\xi})^{n_{B}}\left[\operatorname{det} \widehat{\Psi}_{0}^{(c \bar{c})}\right]^{2}\left(\frac{\xi}{4} r\right)^{n_{U}} \operatorname{det} \widehat{\Psi}_{0}^{(\lambda)}+\mathcal{O}(\nu)
\end{aligned}
$$

Thus,

$$
\frac{1}{\nu^{n_{U}}} \frac{\operatorname{det} \Psi_{0, \text { reg, BG,alt }}^{(S \varphi)}(\nu ; r \rightarrow \infty)}{\operatorname{det} \widehat{\Psi}_{0, \mathrm{reg}, \mathrm{BG}}^{(S \varphi)}(\nu ; r \rightarrow \infty)}=\left(-\frac{1}{2 r_{\infty}^{3}}\right)^{n_{U}} \frac{\operatorname{det} \mathcal{K}_{\mathrm{alt}}}{\operatorname{det} W_{U}^{\prime}} \frac{\operatorname{det} \Psi_{0, \text { alt }}^{(\lambda)}}{\operatorname{det} \widehat{\Psi}_{0}^{(\lambda)}}\left(\frac{\operatorname{det} \Psi_{0, \text { alt }}^{(c \bar{c})}}{\operatorname{det} \widehat{\Psi}_{0}^{(c \bar{c})}}\right)^{2}+\mathcal{O}(\nu),
$$


and

$$
\lim _{\nu \rightarrow 0} \frac{1}{\nu^{n_{U}}} \frac{\operatorname{det} \Psi_{0, \text { reg,BG }}^{(S \varphi)}(\nu ; r \rightarrow \infty)}{\operatorname{det} \widehat{\Psi}_{0, \text { reg, }, \mathrm{BG}}^{(S \varphi)}(\nu ; r \rightarrow \infty)}=\left(-\frac{1}{2 r_{\infty}^{3}}\right)^{n_{U}} \frac{\operatorname{det} \mathcal{K}}{\operatorname{det} W_{U}^{\prime}} \frac{\operatorname{det} \Psi_{0}^{(\lambda)}}{\operatorname{det} \widehat{\Psi}_{0}^{(\lambda)}}\left(\frac{\operatorname{det} \Psi_{0}^{(c \bar{c})}}{\operatorname{det} \widehat{\Psi}_{0}^{(c \bar{c})}}\right)^{2} .
$$

From eqs. (4.33), (4.34) and (4.35),

$$
\lim _{r \rightarrow 0} \frac{\operatorname{det} \Psi_{0}^{(S \varphi)}(r)}{\operatorname{det} \widehat{\Psi}_{0}^{(S \varphi)}(r)}=\frac{\sqrt{\operatorname{det} M_{0}^{T} M_{0}}}{\operatorname{det} \widehat{W}},
$$

independently of the choice of the guage fixing. Based on the above results, eq. (5.51) is obtained.

\section{B Use of alternative fluctuation opeartors}

In this appendix, we justify the use of the alternative fluctuation operators in the evaluation of the determinants at $r \rightarrow \infty$.

\section{B.1 General discussion}

\section{B.1.1 Setup}

We compare the determinants of the two functions, $\mathfrak{F}$ and $\mathfrak{F}_{\text {alt }}$, which satisfy

$$
\begin{array}{r}
{\left[-\partial_{r}^{2}-\Lambda(r) \partial_{r}-\delta \Lambda(r) \partial_{r}+\Xi(r)+\delta \Xi(r)\right] \mathfrak{F}(r)=0,} \\
{\left[-\partial_{r}^{2}-\Lambda(r) \partial_{r}+\Xi(r)\right] \mathfrak{F}_{\text {alt }}(r)=0,}
\end{array}
$$

where $\Lambda, \delta \Lambda, \Xi, \delta \Xi, \mathfrak{F}$ and $\mathfrak{F}_{\text {alt }}$ are $n \times n$ matrices. The support of $\delta \Lambda(r)$ and $\delta \Xi(r)$ is $r>r_{*}$ and we take

$$
\mathfrak{F}(r)=\mathfrak{F}_{\text {alt }}(r) \quad: \quad r<r_{*} .
$$

We assume that there exist constants, $C_{\mathfrak{F}}, C_{\delta \Lambda}$ and $C_{\delta \Xi}$, such that

$$
\left\|\mathfrak{F}_{\text {alt }}^{\prime}(r) \mathfrak{F}_{\text {alt }}^{-1}(r)\right\|<C_{\mathfrak{F}}, \quad\|\delta \Lambda(r)\|<\frac{C_{\delta \Lambda}}{r^{3}}, \quad\|\delta \Xi(r)\|<\frac{C_{\delta \Xi}}{r^{3}},
$$

where $\|A\|$ is the induced (spectral) norm of the real matrix $A$, which is defined as

$$
\|A\|=\max _{x \in \mathbb{R}^{n},|x| \neq 0} \frac{|A x|}{|x|},
$$

with $|x|=\sqrt{\sum_{i} x_{i}^{2}}$. In addition, we assume

$$
\mu\left(-\mathfrak{F}_{\text {alt }}^{\prime}(r) \mathfrak{F}_{\text {alt }}^{-1}(r)\right) \leq 0, \quad \mu(-\Lambda(r))<-\frac{k}{r},
$$

where $k>2$ is a non-integer constant and $\mu(A)$ is the logarithmic norm of matrix $A$, which is defined as

$$
\mu(A)=\lim _{h \rightarrow 0^{+}} \frac{\|I+h A\|-1}{h} .
$$

Notice that $\mu(A)$ gives the largest eigenvalue of $\frac{1}{2}\left(A+A^{T}\right)$.

The goal of this subsection is to show that the following quantity has an upper bound:

$$
\lim _{r \rightarrow \infty}\left|\ln \frac{\operatorname{det} \mathfrak{F}(r)}{\operatorname{det} \mathfrak{F}_{\text {alt }}(r)}\right| \text {. }
$$




\section{B.1.2 Recursive formula}

Let us construct a formal solution of eq. (B.1) using $\mathfrak{F}_{\text {alt }}$. We express $\mathfrak{F}$ as

$$
\mathfrak{F}(r)=\mathfrak{F}_{\text {alt }}(r) \mathcal{P} \exp \left[\int_{r_{*}}^{r} d s \mathfrak{F}_{\text {alt }}^{-1}(s) \Theta(s) \mathfrak{F}_{\text {alt }}(s)\right],
$$

where $\Theta$ is a function satisfying

$$
\Theta^{\prime}+\left(\mathfrak{F}_{\text {alt }}^{\prime} \mathfrak{F}_{\text {alt }}^{-1}+\Lambda\right) \Theta+\Theta \mathfrak{F}_{\text {alt }}^{\prime} \mathfrak{F}_{\text {alt }}^{-1}=\delta \Xi-\delta \Lambda \mathfrak{F}_{\text {alt }}^{\prime} \mathfrak{F}_{\text {alt }}^{-1}-\Theta^{2}-\delta \Lambda \Theta .
$$

Here, the path-ordered exponential of matrix $A(s)$ is defined as ${ }^{12}$

$$
\mathcal{P} \exp \left[\int_{r_{\mathrm{i}}}^{r_{\mathrm{f}}} d s A(s)\right]=1+\sum_{p=1}^{\infty} \int_{r_{\mathrm{i}}}^{r_{\mathrm{f}}} d s_{p} \int_{r_{\mathrm{i}}}^{s_{p}} d s_{p-1} \cdots \int_{r_{\mathrm{i}}}^{s_{2}} d s_{1} A\left(s_{p}\right) A\left(s_{p-1}\right) \cdots A\left(s_{1}\right) .
$$

Treating $\delta \Lambda$ and $\delta \Xi$ as perturbations, we expand $\Theta$ as

$$
\Theta=\Theta^{(1)}+\Theta^{(2)}+\cdots
$$

where $\Theta^{(p)}$ is the $p$-th order term with respect to fluctuations. Formally, we obtain

$$
\Theta^{(p)}(r)=\int_{r_{*}}^{r} d s \mathcal{P} \exp \left[\int_{s}^{r} d t\left(-\mathfrak{F}_{\text {alt }}^{\prime}(t) \mathfrak{F}_{\text {alt }}^{-1}(t)-\Lambda(t)\right)\right] \Delta^{(p)}(s) \mathcal{P} \exp \left[\int_{r}^{s} d t \mathfrak{F}_{\text {alt }}^{\prime}(t) \mathfrak{F}_{\text {alt }}^{-1}(t)\right],
$$

where

$$
\Delta^{(p)}=\left\{\begin{array}{ll}
\delta \Xi-\delta \Lambda \mathfrak{F}_{\text {alt }}^{\prime} \mathfrak{F}_{\text {alt }}^{-1} & : p=1 \\
-\delta \Lambda \Theta^{(p-1)}-\sum_{i=1}^{p-1} \Theta^{(i)} \Theta^{(p-i)} & : p>1
\end{array} .\right.
$$

This recursive formula can be solved order by order and we can obtain $\Theta$ if the sum over $p$ converges.

\section{B.1.3 Error evaluation formula}

Let us first evaluate the right path-ordered exponential in eq. (B.12). Using eq. (B.5) as well as

$$
\mathcal{P} \exp \left[\int_{r}^{s} d t \mathfrak{F}_{\text {alt }}^{\prime}(t) \mathfrak{F}_{\text {alt }}^{-1}(t)\right] y_{R}(r)=y_{R}(s),
$$

the following relation holds:

$$
\left\|\mathcal{P} \exp \left[\int_{r}^{s} d t \mathfrak{F}_{\text {alt }}^{\prime}(t) \mathfrak{F}_{\text {alt }}^{-1}(t)\right]\right\|=\max _{\left|y_{R}(r)\right| \neq 0} \frac{\left|y_{R}(s)\right|}{\left|y_{R}(r)\right|},
$$

\footnotetext{
${ }^{12}$ One may also write the path-ordered exponential as

$$
\mathcal{P} \exp \left[\int_{r_{\mathrm{i}}}^{r_{\mathrm{f}}} d s A(s)\right]=\lim _{N \rightarrow \infty} e^{A\left(r_{\mathrm{f}}\right) \delta s} e^{A\left(r_{\mathrm{f}}-\delta s\right) \delta s} e^{A\left(r_{\mathrm{f}}-2 \delta s\right) \delta s} \cdots e^{A\left(r_{\mathrm{f}}-N \delta s\right) \delta s},
$$
}

with

$$
\delta s=\frac{r_{\mathrm{f}}-r_{\mathrm{i}}}{N} .
$$


where $y_{R}(t)$ satisfies

$$
y_{R}^{\prime}(t)=\mathfrak{F}_{\text {alt }}^{\prime}(t) \mathfrak{F}_{\text {alt }}^{-1}(t) y_{R}(t)
$$

Then,

$$
\begin{aligned}
D^{-}\left|y_{R}(t)\right| & =\lim _{h \rightarrow 0^{+}} \frac{\left|y_{R}(t)\right|-\left|y_{R}(t-h)\right|}{h} \\
& =\lim _{h \rightarrow 0^{+}} \frac{\left|y_{R}(t)\right|-\left|y_{R}(t)-h \mathfrak{F}_{\text {alt }}^{\prime}(t) \mathfrak{F}_{\text {alt }}^{-1}(t) y_{R}(t)\right|}{h} \\
& \geq-\mu\left(-\mathfrak{F}_{\text {alt }}^{\prime}(t) \mathfrak{F}_{\text {alt }}^{-1}(t)\right)\left|y_{R}(t)\right|,
\end{aligned}
$$

where $D^{-}$is the left-hand derivative. After integration, we obtain

$$
\ln \frac{\left|y_{R}(r)\right|}{\left|y_{R}(s)\right|} \geq-\int_{s}^{r} d t \mu\left(-\mathfrak{F}_{\text {alt }}^{\prime}(t) \mathfrak{F}_{\text {alt }}^{-1}(t)\right) \geq 0
$$

resulting in

$$
\left\|\mathcal{P} \exp \left[\int_{r}^{s} d t \mathfrak{F}_{\text {alt }}^{\prime}(t) \mathfrak{F}_{\text {alt }}^{-1}(t)\right]\right\| \leq 1
$$

Similarly, we evaluate the left path-ordered exponential in eq. (B.12). From eq. (B.5),

$$
\left\|\mathcal{P} \exp \left[\int_{s}^{r} d t\left(-\mathfrak{F}_{\text {alt }}^{\prime}(t) \mathfrak{F}_{\text {alt }}^{-1}(t)-\Lambda(t)\right)\right]\right\|=\max _{\left|y_{L}(s)\right| \neq 0} \frac{\left|y_{L}(r)\right|}{\left|y_{L}(s)\right|}
$$

with

$$
y_{L}^{\prime}(t)=\left(-\mathfrak{F}_{\text {alt }}^{\prime}(t) \mathfrak{F}_{\text {alt }}^{-1}(t)-\Lambda(t)\right) y_{L}(t)
$$

Then, we obtain

$$
\begin{aligned}
D^{+}\left|y_{L}(t)\right| & =\lim _{h \rightarrow 0^{+}} \frac{\left|y_{L}(t+h)\right|-\left|y_{L}(t)\right|}{h} \\
& =\lim _{h \rightarrow 0^{+}} \frac{\left|y_{L}(t)+h\left(-\mathfrak{F}_{\text {alt }}^{\prime}(t) \mathfrak{F}_{\text {alt }}^{-1}(t)-\Lambda(t)\right) y_{L}(t)\right|-\left|y_{L}(t)\right|}{h} \\
& \leq \mu\left(-\mathfrak{F}_{\text {alt }}^{\prime}(t) \mathfrak{F}_{\text {alt }}^{-1}(t)-\Lambda(t)\right)\left|y_{L}(t)\right|,
\end{aligned}
$$

where $D^{+}$is the right-hand derivative, based on which we find

$$
\ln \frac{\left|y_{L}(r)\right|}{\left|y_{L}(s)\right|} \leq \int_{r}^{s} d t \mu\left(-\mathfrak{F}_{\text {alt }}^{\prime}(t) \mathfrak{F}_{\text {alt }}^{-1}(t)\right)+\int_{r}^{s} d t \mu(-\Lambda(t))<-k \ln \left(\frac{r}{s}\right) .
$$

Thus, the following inequality holds:

$$
\left\|\mathcal{P} \exp \left[\int_{s}^{r} d t\left(-\mathfrak{F}_{\text {alt }}^{\prime}(t) \mathfrak{F}_{\text {alt }}^{-1}(t)-\Lambda(t)\right)\right]\right\|<\left(\frac{s}{r}\right)^{k} .
$$

Using eqs. (B.19) and (B.24), we obtain

$$
\left\|\Theta^{(p)}(r)\right\|<\int_{r_{*}}^{r} d s\left(\frac{s}{r}\right)^{k}\left\|\Delta^{(p)}(s)\right\| .
$$


Now, we show that $\left\|\Theta^{(p)}\right\|$ is bounded from above as

$$
\left\|\Theta^{(p)}(r)\right\|<C^{(p)} \frac{r_{*}}{r^{2}}
$$

where $C^{(p)}$ 's are positive constants. Indeed, it is the case for $p=1$ as

$$
\begin{aligned}
\left\|\Theta^{(1)}(r)\right\| & <\int_{r_{*}}^{r} d s\left(\frac{s}{r}\right)^{k}\left\|\Delta^{(1)}(s)\right\| \\
& <\left(C_{\delta \Xi}+C_{\delta \Lambda} C_{\mathfrak{F}}\right) \int_{r_{*}}^{r} d s\left(\frac{s}{r}\right)^{k} \frac{1}{s^{3}} \\
& =\frac{C_{\delta \Xi}+C_{\delta \Lambda} C_{\mathfrak{F}}}{k-2} \frac{1}{r^{2}}\left(1-\frac{r_{*}^{k-2}}{r^{k-2}}\right) \\
& <C^{(1)} \frac{r_{*}}{r^{2}}
\end{aligned}
$$

where

$$
C^{(1)}=\frac{C_{\delta \Xi}+C_{\delta \Lambda} C_{\mathfrak{F}}}{(k-2) r_{*}}
$$

Next, let us assume $\left\|\Theta^{(q)}(r)\right\|<C^{(q)} \frac{r_{*}}{r^{2}}$ for all $q<p$, and show that the inequality (B.26) holds. Indeed,

$$
\begin{aligned}
\left\|\Theta^{(p)}(r)\right\| & <\int_{r_{*}}^{r} d s\left(\frac{s}{r}\right)^{k}\left\|\Delta^{(p)}(s)\right\| \\
& <\int_{r_{*}}^{r} d s\left(\frac{s}{r}\right)^{k}\left(r_{*} \frac{C_{\delta \Lambda} C^{(p-1)}}{s^{5}}+\sum_{i=1}^{p-1} r_{*}^{2} \frac{C^{(i)} C^{(p-i)}}{s^{4}}\right) \\
& =\frac{C_{\delta \Lambda} C^{(p-1)}}{k-4} r_{*}\left(\frac{1}{r^{4}}-\frac{r_{*}^{k-4}}{r^{k}}\right)+\sum_{i=1}^{p-1} \frac{C^{(i)} C^{(p-i)}}{k-3} r_{*}^{2}\left(\frac{1}{r^{3}}-\frac{r_{*}^{k-3}}{r^{k}}\right) \\
& <C^{(p)} \frac{r_{*}}{r^{2}},
\end{aligned}
$$

where

$$
C^{(p)}=\left(\frac{C_{\delta \Lambda}}{r_{*}^{2}} \frac{C^{(p-1)}}{|k-4|}+\sum_{i=1}^{p-1} \frac{C^{(i)} C^{(p-i)}}{|k-3|}\right) .
$$

Thus, the inequality (B.26) is valid for all $p$.

Finally, we evaluate an upper bound on $\|\Theta(r)\|$. Let us define

$$
C^{\text {tot }}=\sum_{p=1}^{\infty} C^{(p)}
$$

Then, if it converges, we get

$$
\|\Theta(r)\|<C^{\text {tot }} \frac{r_{*}}{r^{2}}
$$

In figure 1 , we show the contours of constant $C^{\text {tot }}$, taking $k=2.5$. As we can see from the figure, $C^{\text {tot }}$ is actually convergent for small enough $C^{(1)}$ and $C_{\delta \Lambda} / r_{*}^{2}$, and $C^{\text {tot }}$ approaches zero as $C^{(1)}$ and $C_{\delta \Lambda} / r_{*}^{2}$ go to zero. 


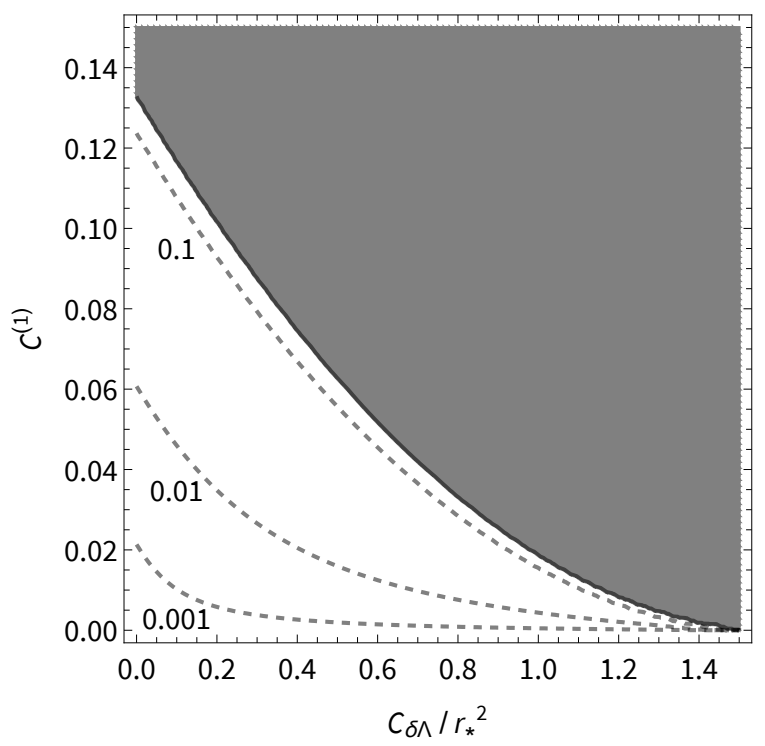

Figure 1. Contours of constant $C^{\text {tot }}$, taking $k=2.5$. The dashed lines indicate $C^{\text {tot }}=0.1,0.01$, and 0.001. In the gray region, $C^{\text {tot }}$ diverges.

Thus, if $C^{\text {tot }}$ converges, we obtain

$$
\lim _{r \rightarrow \infty}\left|\ln \frac{\operatorname{det} \mathfrak{F}(r)}{\operatorname{det} \mathfrak{F}_{\text {alt }}(r)}\right|=\left|\int_{r_{*}}^{\infty} d s \operatorname{Tr} \Theta(s)\right| \leq n \int_{r_{*}}^{\infty} d s\|\Theta(s)\|=n C^{\text {tot }} .
$$

\section{B.2 Alternative fluctuation operators}

Let us consider a one-parameter family of differential equations with different transition point, $r_{*}$, and we denote the solution as $\mathfrak{F}_{\text {alt }}\left(r_{*} ; r\right)$. Then, $C_{\delta \Xi}, C_{\delta \Lambda}$ and $C_{\mathfrak{F}}$ are dependent on $r_{*}$. From the above discussion, sufficient conditions for

$$
\lim _{r_{*} \rightarrow \infty} \lim _{r \rightarrow \infty}\left|\ln \frac{\operatorname{det} \mathfrak{F}(r)}{\operatorname{det} \mathfrak{F}_{\text {alt }}\left(r_{*} ; r\right)}\right|=1,
$$

are given by

$$
\begin{aligned}
\lim _{r_{*} \rightarrow \infty} \frac{C_{\delta \Xi}\left(r_{*}\right)+C_{\delta \Lambda}\left(r_{*}\right) C_{\mathfrak{F}}\left(r_{*}\right)}{r_{*}} & =0, \\
\lim _{r_{*} \rightarrow \infty} \frac{C_{\delta \Lambda}\left(r_{*}\right)}{r_{*}^{2}} & =0 .
\end{aligned}
$$

In this subsection, we give several general remarks. Then, in the following subsections, for $(S L \varphi)$ modes, $(c \bar{c})$ modes and $(\eta \lambda)$ modes, we construct $\mathfrak{F}_{\text {alt }}\left(r_{*} ; r\right)$ explicitly and show that the above conditions as well as eq. (B.6) are satisfied.

\section{B.2.1 Extended fluctuation operators}

Since the conditions (B.4) are sensitive to the behavior of the fluctuation operators around $r \sim r_{*}$, we need to specify their deformation explicitly. 
In particular, the differential equations for $\Psi_{\text {alt }}^{(\eta \lambda)}$ should be well-defined for all $r$, which requires that the decomposition of solutions should be exact for all $r$. The decomposition with the alternative fluctuation operators becomes exact when the following relations are satisfied;

$$
\begin{aligned}
\left(M_{\mathrm{alt}}^{T}\right)^{\prime} M_{\mathrm{alt}} & =M_{\mathrm{alt}}^{T} M_{\mathrm{alt}}^{\prime}, \\
\Omega_{\mathrm{alt}}^{T} & =\Omega_{\mathrm{alt}}, \\
P_{H, \mathrm{alt}}\left(-\Delta_{0} M_{\mathrm{alt}}+\Omega_{\mathrm{alt}} M_{\mathrm{alt}}\right) & =0 .
\end{aligned}
$$

However, it is generally difficult to deform the fluctuation operators keeping these relations. In the following, we show a way to overcome it by extending the fluctuation operators.

We introduce $n_{G}$ spectator scalars and extend $M$ and $\Omega$ as

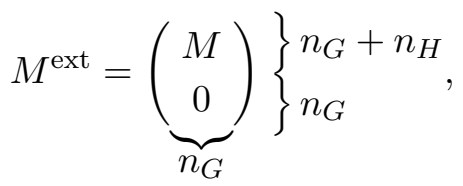

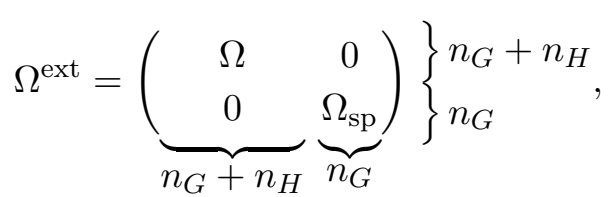

where $\Omega_{\mathrm{sp}}$ is an $n_{G} \times n_{G}$ matrix, which will be determined later. We take the same $\Omega_{\mathrm{sp}}$ for the false vacuum. Thus, the spectator scalars do not affect the ratio of the determinants. We also introduce $P_{G \text {,alt }}^{\text {ext }}$ and $P_{H, \text { alt }}^{\text {ext }}$, which are defined as

$$
\begin{aligned}
& P_{G, \text { alt }}^{\text {ext }}=M_{\text {alt }}^{\text {ext }}\left(M_{\mathrm{alt}}^{\text {ext } T} M_{\mathrm{alt}}^{\text {ext }}\right)^{2} M_{\mathrm{alt}}^{\text {ext } T}, \\
& P_{H, \text { alt }}^{\text {ext }}=I_{\varphi}^{\text {ext }}-P_{G, \text { alt }}^{\text {ext }},
\end{aligned}
$$

where $I_{\varphi}^{\text {ext }}$ is the $\left(n_{\varphi}+n_{G}\right) \times\left(n_{\varphi}+n_{G}\right)$ identity matrix.

We assume that the deformation begins at $r=r_{*}=r_{1}$ and ends at $r=r_{5}$ with $r_{5}-r_{1} \ll r_{*}$. We construct the alternative matrices, $M_{\mathrm{alt}}^{\text {ext }}$ and $\Omega_{\mathrm{alt}}^{\text {ext }}$, which satisfy $M_{\text {alt }}^{\text {ext }}(r)=M^{\text {ext }}(r)$ and $\Omega_{\text {alt }}^{\text {ext }}(r)=\Omega^{\text {ext }}(r)$ for $r<r_{1}$. For $r_{1}<r<r_{5}$, we deform them in the following way.

1. We express $M_{\text {alt }}^{\text {ext }}$ as

$$
M_{\mathrm{alt}}^{\mathrm{ext}}(r)=\left(\begin{array}{c}
M_{\mathrm{alt}}(r) \\
\omega(r)
\end{array}\right),
$$

where $\omega$ expresses the elements for the spectator scalars and $\omega\left(r_{1}\right)=0$. Keeping the following relations:

$$
\begin{aligned}
\Omega_{\mathrm{alt}}^{\mathrm{ext}}= & \Omega^{\mathrm{ext}}-P_{H, \mathrm{alt}}^{\mathrm{ext}}\left[-\Delta_{0} M_{\mathrm{alt}}^{\mathrm{ext}}+\Omega^{\mathrm{ext}} M_{\mathrm{alt}}^{\mathrm{ext}}\right]\left(M_{\mathrm{alt}}^{\mathrm{ext} T} M_{\mathrm{alt}}^{\mathrm{ext}}\right)^{-1} M_{\mathrm{alt}}^{\mathrm{ext} T} \\
& -M_{\mathrm{alt}}^{\mathrm{ext}}\left(M_{\mathrm{alt}}^{\mathrm{ext} T} M_{\mathrm{alt}}^{\mathrm{ext}}\right)^{-1}\left[-\Delta_{0} M_{\mathrm{alt}}^{\mathrm{ext} T}+M_{\mathrm{alt}}^{\mathrm{ext} T} \Omega^{\mathrm{ext}}\right] P_{H, \mathrm{alt}}^{\mathrm{ext}}, \\
\Omega_{\mathrm{sp}}= & \left(\Delta_{0} \omega\right) \omega^{-1},
\end{aligned}
$$

we deform $M_{\text {alt }}^{\text {ext }}$ in the following steps. 
(a) $r_{1}<r<r_{2}$ : Turn on $\omega$ keeping $\omega \propto \omega\left(r_{2}\right)$.

(b) $r_{2}<r<r_{3}$ : Rotate $M_{\text {alt }}$ so that $M_{\text {alt }}^{T}\left(r_{3}\right)=(W, 0)$, where $W$ is a diagonal matrix. During the rotation, we change $\omega$ so that

$$
\omega^{T} \omega^{\prime}-\omega^{\prime T} \omega=M_{\mathrm{alt}}^{\prime T} M_{\mathrm{alt}}-M_{\mathrm{alt}}^{T} M_{\mathrm{alt}}^{\prime}
$$

(c) $r_{3}<r<r_{4}$ : Turn off $\omega$ keeping $\omega \propto \omega\left(r_{3}\right)$.

2. $r_{4}<r<r_{5}$ : Diagonalize $P_{H, \text { alt }}^{\text {ext }} \Omega_{\text {alt }}^{\text {ext }} P_{H \text {,alt }}^{\text {ext }}$.

Here, we take $r_{p+1}-r_{p}$ independently of $r_{*}$. One can easily check that eqs. (B.37), (B.38) and (B.39) hold at each step.

\section{B.2.2 Linear approximation}

To evaluate the effect of the deformation on the functional determinants, we construct each step of the deformation more concretely. Since the difference between the original matrices and the alternative matrices are expected to be very small, we can work in the linear approximation. As in the previous sections, we choose the basis of the fields so that

$$
\begin{aligned}
\lim _{r \rightarrow \infty} M(r) W^{-1}(r) & =\left(\begin{array}{c}
I_{G} \\
0
\end{array}\right), \\
\lim _{r \rightarrow \infty} \Omega(r) & =\left(\begin{array}{cc}
0 & 0 \\
0 & \widehat{m}^{2}
\end{array}\right) .
\end{aligned}
$$

Notice that the first condition is somewhat stronger than eq. (A.6) since some of the elements of $W^{-1}(r)$ diverge as $r \rightarrow \infty$. The existence of such a field basis is guaranteed by the one-to-one correspondence between the massive gauge bosons and the NG bosons.

Since $O_{\varphi}$ and $O_{G}$ are very close to the identity matrices for a large enough $r_{*}$, we approximate them as

$$
\begin{aligned}
& O_{\varphi}\left(r_{*}\right) \simeq I_{\varphi}+\delta_{\varphi}, \\
& O_{G}\left(r_{*}\right) \simeq I_{G}+\delta_{G},
\end{aligned}
$$

where $\delta_{\varphi}$ and $\delta_{G}$ are constant anti-symmetric matrices and satisfy

$$
\left\|\delta_{\varphi}\right\|_{\max }<\delta_{\max }^{2},\left\|\delta_{G}\right\|_{\max }<\delta_{\max }^{2}
$$

with $\|\cdots\|_{\max }$ being the $\max$ norm and $\delta_{\max } \ll 1$.

We also approximate that $M(r)$ and $\Omega(r)$ are almost constant during the deformation and express them as

$$
\begin{aligned}
& M(r) \simeq M_{*}=\left(I_{\varphi}+\delta_{\varphi}\right)\left(\begin{array}{c}
W_{*} \\
0
\end{array}\right)\left(I_{G}-\delta_{G}\right), \\
& \Omega(r) \simeq\left(I_{\varphi}+\delta_{\varphi}\right)\left(\begin{array}{cc}
0 & 0 \\
0 & m^{2}\left(r_{*}\right)
\end{array}\right)\left(I_{\varphi}-\delta_{\varphi}\right),
\end{aligned}
$$


where $W_{*}=W\left(r_{*}\right)$. From eq. (B.48), $\delta_{G}$ should satisfy

$$
\left\|W_{*} \delta_{G} W_{*}^{-1}\right\|_{\max } \ll 1,
$$

for a large enough $r_{*}$.

We define sigmoid functions that have the following features:

$$
\begin{aligned}
\varsigma_{p}(r) & \simeq\left\{\begin{array}{ll}
0 & r \lesssim r_{p} \\
1 & r \gtrsim r_{p+1}
\end{array},\right. \\
\max _{r}\left|\partial_{r}^{n} \varsigma_{p}(r)\right| & \sim\left(r_{p+1}-r_{p}\right)^{-n} .
\end{aligned}
$$

Then, we construct the deformation as follows.

- $r_{1}<r<r_{2}$ : We turn on $\omega$ as

$$
\omega(r)=\varsigma_{1}(r) \delta_{\max }^{1 / 2} W_{*} .
$$

In this step, $M_{\text {alt }}$ and $\Omega_{\text {alt }}^{\text {ext }}$ are constant.

- $r_{2}<r<r_{3}$ : We rotate $M_{\text {alt }}$ and $\omega$ as

$$
\begin{aligned}
M_{\mathrm{alt}}(r) & =\left(I_{\varphi}-\varsigma_{2}(r) \delta_{\varphi}\right) M_{*}\left(I_{G}+\varsigma_{2}(r) \delta_{G}\right), \\
\omega(r) & =\sqrt{\delta_{\max }}\left(I_{\varphi}+\varepsilon_{\varphi}(r)\right) W_{*}\left(1-\varepsilon_{G}(r)\right),
\end{aligned}
$$

where

$$
\begin{aligned}
& \varepsilon_{\varphi}(r)=\frac{\varsigma_{2}(r)}{\delta_{\max }}\left(\begin{array}{ll}
I_{G} & 0
\end{array}\right) \delta_{\varphi}\left(\begin{array}{c}
I_{G} \\
0
\end{array}\right), \\
& \varepsilon_{G}(r)=\frac{\varsigma_{2}(r)}{\delta_{\max }} \delta_{G} .
\end{aligned}
$$

Notice that the elements of $\varepsilon_{\varphi}$ and $\varepsilon_{G}$ are much smaller than one due to eq. (B.52). In this step, $\Omega_{\text {alt }}^{\text {ext }}$ is changed according to eq. (B.45).

- $r_{3}<r<r_{4}$ : We turn off $\omega$ as

$$
\omega(r)=\left(1-\varsigma_{3}(r)\right) \omega\left(r_{3}\right) .
$$

In this step, $M_{\text {alt }}$ and $\Omega_{\text {alt }}^{\text {ext }}$ are constant.

- $r_{4}<r<r_{5}$ : Finally, we rotate $P_{H, \text { alt }}^{\text {ext }} \Omega_{\text {alt }}^{\text {ext }} P_{H, \text { alt }}^{\text {ext }}$ as

$$
P_{H, \text { alt }}^{\text {ext }} \Omega_{\text {alt }}^{\text {ext }} P_{H, \text { alt }}^{\text {ext }}=P_{H, \text { alt }}^{\text {ext }}\left(\begin{array}{cc}
I_{\varphi}-\varsigma_{4}(r) \delta_{\varphi} & 0 \\
0 & I_{G}
\end{array}\right) \Omega_{\text {alt }}^{\text {ext }}\left(\begin{array}{cc}
I_{\varphi}+\varsigma_{4}(r) \delta_{\varphi} & 0 \\
0 & I_{G}
\end{array}\right) P_{H, \text { alt }}^{\text {ext }} .
$$

Notice that we can still use $\delta_{\varphi}$ to diagonalize $\Omega$ since

$$
P_{H, \text { alt }}^{\text {ext }}\left(r_{4}\right) \Omega_{\text {alt }}^{\text {ext }}\left(r_{4}\right) P_{H, \text { alt }}^{\text {ext }}\left(r_{4}\right)=P_{H \text {,alt }}^{\text {ext }}\left(r_{4}\right) \Omega^{\text {ext }}\left(r_{4}\right) P_{H, \text { alt }}^{\text {ext }}\left(r_{4}\right) .
$$

In this step, $M_{\text {alt }}^{\text {ext }}$ is constant. 
Finally, let us discuss the deformation of $V_{H}^{\text {ext }}$, which is defined as

$$
V_{H}^{\mathrm{ext}}=\left(\begin{array}{cc}
V_{H} & 0 \\
0 & I_{G}
\end{array}\right) \equiv\left(u_{1}^{\mathrm{ext}} \cdots u_{n_{H}+n_{G}}^{\mathrm{ext}}\right) .
$$

Its alternative can be defined in the following way. Since the difference between $M^{\text {ext }}$ and $M_{\text {alt }}^{\text {ext }}$ is small, we can construct $\left(n_{H}+n_{G}\right)$ independent vectors as

$$
\tilde{u}_{p, \text { alt }}^{\text {ext }}=\frac{P_{H, \text { alt }}^{\text {ext }} u_{p}^{\text {ext }}}{\left|P_{H, \text { alt }}^{\text {ext }} u_{p}^{\text {ext }}\right|}
$$

Using Gram-Schmidt orthogonalization, we get an orthonormal basis, $u_{p \text {,alt }}^{\text {ext }}$, and define

$$
V_{H, \text { alt }}^{\text {ext }}=\left(u_{1, \text { alt }}^{\text {ext }} \cdots u_{n_{H}+n_{G}, \text { alt }}^{\text {ext }}\right) .
$$

\section{B.3 (SL $\varphi)$ modes}

In the rest of this appendix, we confirm eqs. (B.35) and (B.36) for each mode. We start with the $(S L \varphi)$ modes.

We consider the functions $\mathfrak{F}^{(S L \varphi)}$ and $\mathfrak{F}_{\text {alt }}^{(S L \varphi)}$ which obey

$$
\begin{aligned}
& \left(\begin{array}{ccc}
\sqrt{\xi} I_{G} & 0 & 0 \\
0 & I_{G} & 0 \\
0 & 0 & I_{\varphi}^{\text {ext }}
\end{array}\right) \mathcal{M}_{\ell}^{(S L \varphi) \operatorname{ext}}\left(\begin{array}{ccc}
\sqrt{\xi} I_{G} & 0 & 0 \\
0 & I_{G} & 0 \\
0 & 0 & I_{\varphi}^{\text {ext }}
\end{array}\right) \mathfrak{F}^{(S L \varphi)}=0 \\
& \left(\begin{array}{ccc}
\sqrt{\xi} I_{G} & 0 & 0 \\
0 & I_{G} & 0 \\
0 & 0 & I_{\varphi}^{\text {ext }}
\end{array}\right) \mathcal{M}_{\ell, \text { alt }}^{(S L \varphi) \operatorname{ext}}\left(\begin{array}{ccc}
\sqrt{\xi} I_{G} & 0 & 0 \\
0 & I_{G} & 0 \\
0 & 0 & I_{\varphi}^{\text {ext }}
\end{array}\right) \mathfrak{F}_{\text {alt }}^{(S L \varphi)}=0
\end{aligned}
$$

where the superscript "ext" indicates that the $n_{G}$ spectator scalars are included. Then,

$$
\lim _{r \rightarrow \infty}\left|\ln \frac{\operatorname{det} \Psi_{\ell}^{(S L \varphi)}(r)}{\operatorname{det} \Psi_{\ell, \text { alt }}^{(S L \varphi)}(r)}\right|=\lim _{r \rightarrow \infty}\left|\ln \frac{\operatorname{det} \mathfrak{F}^{(S L \varphi)}(r)}{\operatorname{det} \mathfrak{F}_{\text {alt }}^{(S L \varphi)}(r)}\right| .
$$

Here, we have ignored $\omega$, which is non-vanishing only around $r \sim r_{*}$. One can show that its effect disappears as $r_{*} \rightarrow \infty$.

Then, we can show the following.

- All of the eigenvalues of $\left(\Lambda+\Lambda^{T}\right) / 2$ are $3 / r$ and hence $\mu(-\Lambda)=-3 / r$.

- The fluctuation operator at $r \rightarrow \infty$ has the same number of increasing solutions and deceasing solutions. If there are no zero modes, $\mathfrak{F}_{\text {alt }}^{(S L \varphi)}$ contains all of the increasing

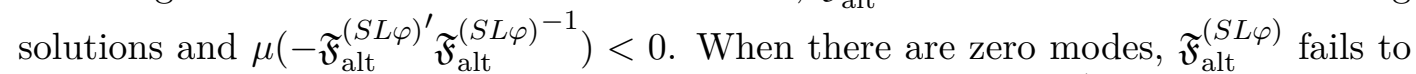
contain some of the increasing solutions and $\mu\left(-\mathfrak{F}_{\text {alt }}^{(S L \varphi)^{\prime}} \mathfrak{F}_{\text {alt }}^{(S L \varphi)^{-1}}\right)$ becomes positive. However, it is harmless since we use the fluctuation operator with regulator, with which all the solutions are increasing.

- Since the growth of $\mathfrak{F}_{\text {alt }}^{(S L \varphi)}$ is either exponential or power at $r \rightarrow \infty, C_{\mathfrak{F}}$ is finite. 
- Since we can take a smaller $\delta_{\max }$ for a larger $r_{*}$, it is easy to find $C_{\delta \Lambda}$ that satisfies eq. (B.35). Notice that the max norm and the spectral norm of an $n \times n$ matrix, $A$, are related through

$$
\|A\| \leq n\|A\|_{\max }
$$

- As for $\delta \Xi$, we need a careful treatment since it includes $\left(W^{T} W\right)^{-1}$. It appears in $\Omega^{\text {ext }}-\Omega_{\text {alt }}^{\text {ext }}$ and

$$
\delta \Omega^{\mathrm{ext}}=-M_{\mathrm{alt}}^{\mathrm{ext}}\left(M_{\mathrm{alt}}^{\mathrm{ext}, T} M_{\mathrm{alt}}^{\mathrm{ext}}\right)^{-1}\left[-\Delta_{0} M_{\mathrm{alt}}^{\mathrm{ext} T}+M_{\mathrm{alt}}^{\mathrm{ext} T} \Omega_{\mathrm{alt}}^{\mathrm{ext}}\right] .
$$

From eq. (B.45), it can be rewritten as

$$
\delta \Omega^{\mathrm{ext}}=-M_{\mathrm{alt}}^{\mathrm{ext}}\left(M_{\mathrm{alt}}^{\mathrm{ext}, T} M_{\mathrm{alt}}^{\mathrm{ext}}\right)^{-1}\left[-\Delta_{0} M_{\mathrm{alt}}^{\mathrm{ext} T}+M_{\mathrm{alt}}^{\mathrm{ext} T} \Omega^{\mathrm{ext}}\right] P_{G, \mathrm{alt}},
$$

for $r_{1}<r<r_{4}$. It is non-vanishing only when $r_{2}<r<r_{3}$, where

$$
\left[-\Delta_{0} M_{\mathrm{alt}}^{\mathrm{ext}}+\Omega^{\mathrm{ext}} M_{\mathrm{alt}}^{\mathrm{ext}}\right]\left(M_{\mathrm{alt}}^{\mathrm{ext} T} M_{\mathrm{alt}}^{\mathrm{ext}}\right)^{-1} M_{\mathrm{alt}}^{\mathrm{ext} T} \simeq\left(\begin{array}{c}
\varsigma_{2}^{\prime \prime}(r)\left[-\delta_{\varphi}+W \delta_{G} W^{-1}\right] \\
0
\end{array}\right),
$$

and $\Omega^{\text {ext }}-\Omega_{\text {alt }}^{\text {ext }}$ and $\delta \Omega^{\text {ext }}$ are obtained by appropriate projections. Since its elements decrease as $\delta_{\max }$ becomes smaller, we can find $C_{\delta \Xi}$ that satisfies eq. (B.36).

The same discussion applies to $\ell=0$.

\section{B.4 $(c \bar{c})$ modes}

For the ghosts, we introduce $\mathfrak{F}^{(c \bar{c})}$ and $\mathfrak{F}_{\text {alt }}^{(c \bar{c})}$, obeying

$$
\begin{aligned}
& \mathcal{M}_{\ell}^{(c \bar{c}) \operatorname{ext}} \mathfrak{F}^{(c \bar{c})}=0 \\
& \mathcal{M}_{\ell, \text { alt }}^{(c \bar{c}) \operatorname{Fxt}} \mathfrak{F}_{\text {alt }}^{(c \bar{c})}=0 .
\end{aligned}
$$

The discussion is almost the same as in the case of the $(S L \varphi)$ modes, but is much simpler because, for the ghosts, $\delta \Lambda=0$ and

$$
\delta \Xi=\xi\left(M^{\mathrm{ext} T} M^{\mathrm{ext}}-M_{\mathrm{alt}}^{\mathrm{ext} T} M_{\mathrm{alt}}^{\mathrm{ext}}\right) .
$$

\section{B.5 $(\eta \lambda)$ modes}

Let us define

$$
\begin{aligned}
& \mathcal{M}_{\ell}^{(\eta \lambda)}= \\
& \left(\begin{array}{ccc}
W^{-1}\left[-\Delta_{\ell}^{\left(M^{T} M\right)}+2 M^{T} M^{\prime}\left(M^{T} M\right)^{-1} \frac{1}{r^{2}} \partial_{r} r^{2}\right] W & -\frac{2 L}{r} W^{-1} M^{T} V_{H} \\
& \frac{2 L}{r} V_{H}^{T} M^{\prime}\left(M^{T} M\right)^{-1} W & V_{H}^{T}\left[-\Delta_{\ell}^{(\Omega)}-4 M^{\prime}\left(M^{T} M\right)^{-1} M^{\prime T}\right] V_{H}
\end{array}\right),
\end{aligned}
$$


where $\Delta_{\ell}^{\left(M^{T} M\right)} \equiv \Delta_{\ell}-M^{T} M$ and $\Delta_{\ell}^{(\Omega)} \equiv \Delta_{\ell}-\Omega$. Then, we consider the solution of the following equation:

$$
\mathcal{M}_{\ell}^{(\eta \lambda)} \tilde{\Psi}_{\ell}^{(\eta \lambda)}=0
$$

Here, the solution, $\tilde{\Psi}_{\ell}^{(\eta \lambda)}$, with appropriate boundary conditions is related to $\Psi_{\ell}^{(\eta \lambda)}$ as

$$
\left(\begin{array}{cc}
W & 0 \\
0 & I_{H}
\end{array}\right) \tilde{\Psi}_{\ell}^{(\eta \lambda)}=\Psi_{\ell}^{(\eta \lambda)} .
$$

We also define its alternative version and its extension to include the spectator scalars. We introduce $\mathfrak{F}^{(\eta \lambda)}$ and $\mathfrak{F}_{\text {alt }}^{(\eta \lambda)}$ which obey

$$
\begin{aligned}
& \mathcal{M}_{\ell}^{(\eta \lambda) \operatorname{ext}} \mathfrak{F}^{(\eta \lambda)}=0, \\
& \mathcal{M}_{\ell, \text { alt }}^{(\eta \lambda) \operatorname{ext}} \mathfrak{F}_{\text {alt }}^{(\eta \lambda)}=0 .
\end{aligned}
$$

Then,

$$
\lim _{r \rightarrow \infty}\left|\ln \frac{\operatorname{det} \Psi_{\ell}^{(\eta \lambda)}(r)}{\operatorname{det} \Psi_{\ell, \text { alt }}^{(\eta \lambda)}(r)}\right|=\lim _{r \rightarrow \infty}\left|\ln \frac{\operatorname{det} \mathfrak{F}^{(\eta \lambda)}(r)}{\operatorname{det} \mathfrak{F}_{\text {alt }}^{(\eta \lambda)}(r)}\right| .
$$

We can show the following.

- Since $\Lambda$ is diagonal with the diagonal components $3 / r, \mu(-\Lambda)=-3 / r$.

- From eq. (A.20) and the divergent behavior of $W_{U}^{-1}, \mathfrak{F}_{\text {alt }}^{(\eta \lambda)}$ contains only the increasing solutions. Thus, $\mu\left(-\mathfrak{F}^{(\eta \lambda)^{\prime}}\right.$ alt $\left.\mathfrak{F}_{\text {alt }}^{(\eta \lambda)^{-1}}\right)<0$.

- Since $\mathfrak{F}_{\text {alt }}^{(\eta \lambda)}$ grows exponentially, $C_{\mathfrak{F}}$ is finite.

- Just like the $(S L \varphi)$ modes, substituting the formulas of the linear approximation, we can find $C_{\delta \Lambda}$ and $C_{\delta \Xi}$ that satisfy eqs. (B.35) and (B.36).

The same discussion applies to $\ell=0$.

\section{C $\overline{\mathrm{MS}}$ Counterterms}

In this appendix, we give the explicit form of the counterterms in the $\overline{\mathrm{MS}}$ scheme.

We define mass squared matrices of gauge bosons and scalars in the false vacuum as

$$
\begin{aligned}
\widehat{m}_{A}^{2} & \equiv \widehat{M}^{T} \widehat{M}, \\
\widehat{m}_{\varphi}^{2} & \equiv \widehat{\Omega}+\widehat{M} \widehat{M}^{T},
\end{aligned}
$$

both of which are diagonal, and the Fourier transformation of mass squared difference

$$
\begin{aligned}
\widetilde{\delta m_{A}^{2}}(k) & =\int d^{4} x\left[M^{T}(r) M(r)-\widehat{m}_{A}^{2}\right] e^{-i k x}, \\
\widetilde{\delta m_{\varphi}^{2}}(k) & =\int d^{4} x\left[\Omega(r)+M(r) M^{T}(r)-m_{\varphi}^{2}\right] e^{-i k x}, \\
\widetilde{M}(k) & =\int d^{4} x M(r) e^{-i k x} .
\end{aligned}
$$


Note that $M$ and $\Omega$ only depend on $r=\sqrt{x_{\mu} x_{\mu}}$ due to the spherical symmetry of the bounce. Accordingly, all of the Fourier transformation given above are functions only of $k \equiv \sqrt{k_{\mu} k_{\mu}}$. Furthermore, $\widetilde{\delta m_{A}^{2}}$ and $\widetilde{\delta m_{\varphi}^{2}}$ are symmetric matrices.

After a straightforward calculation, we obtain

$$
\begin{aligned}
s_{1, \overline{\mathrm{MS}}}^{\left(A_{\mu} \varphi\right)}= & 2 \mathcal{I}_{1}\left(\widehat{m}_{A}^{2}, \widetilde{\delta m_{A}^{2}}\right)+\frac{1}{2} \mathcal{I}_{1}\left(\widehat{m}_{\varphi}^{2}, \widetilde{\delta m_{\varphi}^{2}}\right)+\frac{1}{16 \pi^{2}} \operatorname{Tr}\left[\widehat{m}_{A}^{2} \widetilde{\delta m_{A}^{2}}(0)\right], \\
s_{1, \overline{\mathrm{MS}}}^{(c \bar{c})}= & -\mathcal{I}_{1}\left(\widehat{m}_{A}^{2}, \widetilde{\delta m_{A}^{2}}\right), \\
s_{2, \overline{\mathrm{MS}}}^{\left(A_{\mu} \varphi\right)}= & -\mathcal{I}_{2}\left(\widehat{m}_{A}^{2}, \widehat{m}_{A}^{2}, \widetilde{\delta m_{A}^{2}}\right)-2 \mathcal{I}_{2}\left(\widehat{m}_{A}^{2}, \widehat{m}_{\varphi}^{2}, k \widetilde{M}\right)-\frac{1}{4} \mathcal{I}_{2}\left(\widehat{m}_{\varphi}^{2}, \widehat{m}_{\varphi}^{2}, \widetilde{\delta m_{\varphi}^{2}}\right) \\
& +\frac{1}{256 \pi^{4}} \int_{0}^{\infty} d k k^{3} \operatorname{Tr}\left[\widetilde{\delta m_{A}^{2}}(k) \widetilde{\delta m_{A}^{2}}(k)\right], \\
s_{2, \overline{\mathrm{MS}}}^{(c \bar{c})}= & \frac{1}{2} \mathcal{I}_{2}\left(\widehat{m}_{A}^{2}, \widehat{m}_{A}^{2}, \widetilde{\delta m_{A}^{2}}\right),
\end{aligned}
$$

where

$$
\begin{aligned}
\mathcal{I}_{1}\left(m^{2}, \widetilde{\delta m^{2}}\right) & =-\sum_{i}\left[\widetilde{\delta m^{2}}(0)\right]_{i i} \frac{1}{16 \pi^{2}} A_{0, \overline{\mathrm{MS}}}\left(\left[m^{2}\right]_{i i}\right), \\
\mathcal{I}_{2}\left(m_{1}^{2}, m_{2}^{2}, \widetilde{\delta m^{2}}\right) & =\sum_{i j} \frac{1}{128 \pi^{4}} \int_{0}^{\infty} d k k^{3}\left[\widetilde{\delta m^{2}}(k)\right]_{i j}\left[\widetilde{\delta m^{2}}(k)\right]_{i j} B_{0, \overline{\mathrm{MS}}}\left(k,\left[m_{1}^{2}\right]_{i i},\left[m_{2}^{2}\right]_{j j}\right) .
\end{aligned}
$$

Renormalizing the divergence in the $\overline{\mathrm{MS}}$ scheme, we can evaluate the one-point function $A_{0, \overline{\mathrm{MS}}}$ and the two-point function $B_{0, \overline{\mathrm{MS}}}$ as [62]

$$
\begin{aligned}
A_{0, \overline{\mathrm{MS}}}\left(m^{2}\right)=\lim _{\delta \rightarrow+0} m^{2}\left(1+\ln \frac{\mu^{2}}{m^{2}-i \delta}\right), \\
B_{0, \overline{\mathrm{MS}}}\left(k, m^{2}, M^{2}\right)=\lim _{\delta \rightarrow+0}\left[2+x_{1}(\delta) \ln \frac{x_{1}(\delta)-1}{x_{1}(\delta)}+x_{2}(\delta) \ln \frac{x_{2}(\delta)-1}{x_{2}(\delta)}\right. \\
\left.-\ln \left(1-x_{1}(\delta)\right)-\ln \left(x_{2}(\delta)-1\right)+\ln \left(\frac{\mu^{2}}{k^{2}}\right)\right],
\end{aligned}
$$

where $\mu$ is the renormalization scale while $x_{1}(\delta)$ and $x_{2}(\delta)$ are solutions of the following quadratic equation:

$$
-k^{2} x^{2}+\left(k^{2}-m^{2}+M^{2}\right) x+m^{2}-i \delta=0 .
$$


Open Access. This article is distributed under the terms of the Creative Commons Attribution License (CC-BY 4.0), which permits any use, distribution and reproduction in any medium, provided the original author(s) and source are credited.

\section{References}

[1] G. Isidori, G. Ridolfi and A. Strumia, On the metastability of the standard model vacuum, Nucl. Phys. B 609 (2001) 387 [hep-ph/0104016] [InSPIRE].

[2] P.B. Arnold and S. Vokos, Instability of hot electroweak theory: bounds on $m_{H}$ and $m_{t}$, Phys. Rev. D 44 (1991) 3620 [INSPIRE].

[3] J.R. Espinosa and M. Quirós, Improved metastability bounds on the standard model Higgs mass, Phys. Lett. B 353 (1995) 257 [hep-ph/9504241] [INSPIRE].

[4] N. Arkani-Hamed, S. Dubovsky, L. Senatore and G. Villadoro, (No) Eternal Inflation and Precision Higgs Physics, JHEP 03 (2008) 075 [arXiv:0801.2399] [InSPIRE].

[5] J. Elias-Miro, J.R. Espinosa, G.F. Giudice, G. Isidori, A. Riotto and A. Strumia, Higgs mass implications on the stability of the electroweak vacuum, Phys. Lett. B 709 (2012) 222 [arXiv:1112.3022] [INSPIRE].

[6] A.D. Plascencia and C. Tamarit, Convexity, gauge-dependence and tunneling rates, JHEP 10 (2016) 099 [arXiv: 1510.07613] [inSPIRE].

[7] L. Di Luzio, G. Isidori and G. Ridolfi, Stability of the electroweak ground state in the Standard Model and its extensions, Phys. Lett. B 753 (2016) 150 [arXiv:1509.05028] [INSPIRE].

[8] J.R. Espinosa, M. Garny, T. Konstandin and A. Riotto, Gauge-Independent Scales Related to the Standard Model Vacuum Instability, Phys. Rev. D 95 (2017) 056004 [arXiv:1608.06765] [INSPIRE].

[9] Z. Lalak, M. Lewicki and P. Olszewski, Gauge fixing and renormalization scale independence of tunneling rate in Abelian Higgs model and in the standard model, Phys. Rev. D 94 (2016) 085028 [arXiv: 1605.06713] [INSPIRE].

[10] A. Andreassen, W. Frost and M.D. Schwartz, Scale Invariant Instantons and the Complete Lifetime of the Standard Model, Phys. Rev. D 97 (2018) 056006 [arXiv:1707.08124] [INSPIRE].

[11] S. Chigusa, T. Moroi and Y. Shoji, State-of-the-Art Calculation of the Decay Rate of Electroweak Vacuum in the Standard Model, Phys. Rev. Lett. 119 (2017) 211801 [arXiv: 1707.09301] [INSPIRE].

[12] S. Chigusa, T. Moroi and Y. Shoji, Decay Rate of Electroweak Vacuum in the Standard Model and Beyond, Phys. Rev. D 97 (2018) 116012 [arXiv:1803.03902] [INSPIRE].

[13] N. Cabibbo, L. Maiani, G. Parisi and R. Petronzio, Bounds on the Fermions and Higgs Boson Masses in Grand Unified Theories, Nucl. Phys. B 158 (1979) 295 [InSPIRE].

[14] P.Q. Hung, Vacuum Instability and New Constraints on Fermion Masses, Phys. Rev. Lett. 42 (1979) 873 [INSPIRE].

[15] M. Lindner, M. Sher and H.W. Zaglauer, Probing Vacuum Stability Bounds at the Fermilab Collider, Phys. Lett. B 228 (1989) 139 [INSPIRE]. 
[16] C. Ford, D.R.T. Jones, P.W. Stephenson and M.B. Einhorn, The Effective potential and the renormalization group, Nucl. Phys. B 395 (1993) 17 [hep-lat/9210033] [INSPIRE].

[17] J.A. Casas, J.R. Espinosa and M. Quirós, Improved Higgs mass stability bound in the standard model and implications for supersymmetry, Phys. Lett. B 342 (1995) 171 [hep-ph/9409458] [INSPIRE].

[18] J.A. Casas, J.R. Espinosa and M. Quirós, Standard model stability bounds for new physics within LHC reach, Phys. Lett. B 382 (1996) 374 [hep-ph/9603227] [inSPIRE].

[19] M.B. Einhorn and D.R. Jones, The Effective potential, the renormalisation group and vacuum stability, JHEP 04 (2007) 051 [hep-ph/0702295] [INSPIRE].

[20] J. Ellis, J.R. Espinosa, G.F. Giudice, A. Hoecker and A. Riotto, The Probable Fate of the Standard Model, Phys. Lett. B 679 (2009) 369 [arXiv:0906.0954] [INSPIRE].

[21] G. Degrassi et al., Higgs mass and vacuum stability in the Standard Model at NNLO, JHEP 08 (2012) 098 [arXiv: 1205.6497] [INSPIRE].

[22] S. Alekhin, A. Djouadi and S. Moch, The top quark and Higgs boson masses and the stability of the electroweak vacuum, Phys. Lett. B 716 (2012) 214 [arXiv:1207.0980] [InSPIRE].

[23] F. Bezrukov, M.Y. Kalmykov, B.A. Kniehl and M. Shaposhnikov, Higgs Boson Mass and New Physics, JHEP 10 (2012) 140 [arXiv:1205.2893] [InSPIRE].

[24] A. Andreassen, W. Frost and M.D. Schwartz, Consistent Use of the Standard Model Effective Potential, Phys. Rev. Lett. 113 (2014) 241801 [arXiv: 1408.0292] [INSPIRE].

[25] L. Di Luzio and L. Mihaila, On the gauge dependence of the Standard Model vacuum instability scale, JHEP 06 (2014) 079 [arXiv: 1404.7450] [INSPIRE].

[26] A.V. Bednyakov, B.A. Kniehl, A.F. Pikelner and O.L. Veretin, Stability of the Electroweak Vacuum: Gauge Independence and Advanced Precision, Phys. Rev. Lett. 115 (2015) 201802 [arXiv: 1507.08833] [INSPIRE].

[27] S.R. Coleman, The Fate of the False Vacuum. 1. Semiclassical Theory, Phys. Rev. D 15 (1977) 2929 [Erratum ibid. 16 (1977) 1248] [INSPIRE].

[28] C.G. Callan Jr. and S.R. Coleman, The Fate of the False Vacuum. 2. First Quantum Corrections, Phys. Rev. D 16 (1977) 1762 [INSPIRE].

[29] M. Endo, T. Moroi, M.M. Nojiri and Y. Shoji, Renormalization-Scale Uncertainty in the Decay Rate of False Vacuum, JHEP 01 (2016) 031 [arXiv:1511.04860] [INSPIRE].

[30] S. Oda, Y. Shoji and D.-S. Takahashi, High Scale Validity of the DFSZ Axion Model with Precision, JHEP 03 (2020) 011 [arXiv: 1912.01147] [INSPIRE].

[31] N.K. Nielsen, On the Gauge Dependence of Spontaneous Symmetry Breaking in Gauge Theories, Nucl. Phys. B 101 (1975) 173 [INSPIRE].

[32] R. Fukuda and T. Kugo, Gauge Invariance in the Effective Action and Potential, Phys. Rev. D 13 (1976) 3469 [INSPIRE].

[33] M. Endo, T. Moroi, M.M. Nojiri and Y. Shoji, On the Gauge Invariance of the Decay Rate of False Vacuum, Phys. Lett. B 771 (2017) 281 [arXiv:1703.09304] [INSPIRE].

[34] M. Endo, T. Moroi, M.M. Nojiri and Y. Shoji, False Vacuum Decay in Gauge Theory, JHEP 11 (2017) 074 [arXiv:1704.03492] [INSPIRE]. 
[35] V. Branchina, F. Contino and P.M. Ferreira, Electroweak vacuum lifetime in two Higgs doublet models, JHEP 11 (2018) 107 [arXiv: 1807.10802] [INSPIRE].

[36] M.E. Krauss, T. Opferkuch and F. Staub, The Ultraviolet Landscape of Two-Higgs Doublet Models, Eur. Phys. J. C 78 (2018) 1020 [arXiv:1807.07581] [inSPIRE].

[37] P. Basler, P.M. Ferreira, M. Mühlleitner and R. Santos, High scale impact in alignment and decoupling in two-Higgs doublet models, Phys. Rev. D 97 (2018) 095024 [arXiv:1710.10410] [INSPIRE].

[38] N. Chakrabarty and B. Mukhopadhyaya, High-scale validity of a two Higgs doublet scenario: metastability included, Eur. Phys. J. C 77 (2017) 153 [arXiv:1603.05883] [INSPIRE].

[39] E. Bagnaschi, F. Brümmer, W. Buchmüller, A. Voigt and G. Weiglein, Vacuum stability and supersymmetry at high scales with two Higgs doublets, JHEP 03 (2016) 158 [arXiv: 1512.07761] [INSPIRE].

[40] D. Chowdhury and O. Eberhardt, Global fits of the two-loop renormalized Two-Higgs-Doublet model with soft $Z_{2}$ breaking, JHEP 11 (2015) 052 [arXiv:1503.08216] [INSPIRE].

[41] P. Ferreira, H.E. Haber and E. Santos, Preserving the validity of the Two-Higgs Doublet Model up to the Planck scale, Phys. Rev. D 92 (2015) 033003 [Erratum ibid. 94 (2016) 059903] [arXiv: 1505.04001] [INSPIRE].

[42] D. Das and I. Saha, Search for a stable alignment limit in two-Higgs-doublet models, Phys. Rev. D 91 (2015) 095024 [arXiv: 1503.02135] [INSPIRE].

[43] N. Chakrabarty, U.K. Dey and B. Mukhopadhyaya, High-scale validity of a two-Higgs doublet scenario: a study including LHC data, JHEP 12 (2014) 166 [arXiv:1407.2145] [INSPIRE].

[44] J. Elias-Miro, J.R. Espinosa, G.F. Giudice, H.M. Lee and A. Strumia, Stabilization of the Electroweak Vacuum by a Scalar Threshold Effect, JHEP 06 (2012) 031 [arXiv:1203.0237] [INSPIRE].

[45] O. Lebedev, On Stability of the Electroweak Vacuum and the Higgs Portal, Eur. Phys. J. C 72 (2012) 2058 [arXiv:1203.0156] [INSPIRE].

[46] G.M. Pruna and T. Robens, Higgs singlet extension parameter space in the light of the LHC discovery, Phys. Rev. D 88 (2013) 115012 [arXiv:1303.1150] [INSPIRE].

[47] R. Costa, A.P. Morais, M.O.P. Sampaio and R. Santos, Two-loop stability of a complex singlet extended Standard Model, Phys. Rev. D 92 (2015) 025024 [arXiv:1411.4048] [INSPIRE].

[48] V. Branchina and E. Messina, Stability, Higgs Boson Mass and New Physics, Phys. Rev. Lett. 111 (2013) 241801 [arXiv: 1307.5193] [INSPIRE].

[49] V. Branchina, E. Messina and A. Platania, Top mass determination, Higgs inflation, and vacuum stability, JHEP 09 (2014) 182 [arXiv:1407.4112] [INSPIRE].

[50] V. Branchina, E. Messina and M. Sher, Lifetime of the electroweak vacuum and sensitivity to Planck scale physics, Phys. Rev. D 91 (2015) 013003 [arXiv:1408.5302] [InSPIRE].

[51] V. Branchina, E. Messina and D. Zappala, Impact of Gravity on Vacuum Stability, EPL 116 (2016) 21001 [arXiv: 1601.06963] [INSPIRE].

[52] A. Salvio, A. Strumia, N. Tetradis and A. Urbano, On gravitational and thermal corrections to vacuum decay, JHEP 09 (2016) 054 [arXiv:1608.02555] [INSPIRE]. 
[53] A. Joti et al., (Higgs) vacuum decay during inflation, JHEP 07 (2017) 058 [arXiv: 1706.00792] [INSPIRE].

[54] S.R. Coleman, V. Glaser and A. Martin, Action Minima Among Solutions to a Class of Euclidean Scalar Field Equations, Commun. Math. Phys. 58 (1978) 211 [INSPIRE].

[55] K. Blum, M. Honda, R. Sato, M. Takimoto and K. Tobioka, $O(N)$ Invariance of the Multi-Field Bounce, JHEP 05 (2017) 109 [Erratum ibid. 06 (2017) 060] [arXiv:1611.04570] [INSPIRE].

[56] A. Kusenko, K.-M. Lee and E.J. Weinberg, Vacuum decay and internal symmetries, Phys. Rev. D 55 (1997) 4903 [hep-th/9609100] [INSPIRE].

[57] I.M. Gelfand and A.M. Yaglom, Integration in functional spaces and it applications in quantum physics, J. Math. Phys. 1 (1960) 48 [INSPIRE].

[58] R.F. Dashen, B. Hasslacher and A. Neveu, Nonperturbative Methods and Extended Hadron Models in Field Theory 1. Semiclassical Functional Methods, Phys. Rev. D 10 (1974) 4114 [INSPIRE].

[59] S. Coleman, Aspects of Symmetry: Selected Erice Lectures, Cambridge University Press, Cambridge, U.K. (1985) [DOI] [INSPIRE].

[60] K. Kirsten and A.J. McKane, Functional determinants by contour integration methods, Annals Phys. 308 (2003) 502 [math-ph/0305010] [INSPIRE].

[61] K. Kirsten and A.J. McKane, Functional determinants for general Sturm-Liouville problems, J. Phys. A 37 (2004) 4649 [math-ph/0403050] [InSPIRE].

[62] G. Passarino and M.J.G. Veltman, One Loop Corrections for $e^{+} e^{-}$Annihilation Into $\mu^{+} \mu^{-}$ in the Weinberg Model, Nucl. Phys. B 160 (1979) 151 [INSPIRE]. 